\title{
MULTIDISCIPLINARY AIRCRAFT CONCEPTUAL DESIGN OPTIMIZATION CONSIDERING FIDELITY UNCERTAINTIES
}

\author{
by \\ Daniel Neufeld \\ Bachelor of Engineering, Department of Aerospace Engineering \\ Ryerson University, 2003 \\ Master of Applied Science, Department of Mechanical Engineering \\ Ryerson University, 2005
}

\author{
A dissertation exhibition \\ presented to Ryerson University \\ in partial fulfilment of the degree of \\ Doctorate of Applied Science \\ in the program of \\ Aerospace Engineering
}

Toronto, Ontario, Canada, 2010

(C) Daniel Neufeld 2010 
I hereby declare that I am the sole author of this dissertation.

I authorize Ryerson University to lend this dissertation to other institutions or individuals for scholarly research.

I further authorize Ryerson University to reproduce this dissertation by photocopying or by other means, in total or in part, at the request of other institutions or individuals for scholarly research. 


\title{
MULTIDISCIPLINARY AIRCRAFT CONCEPTUAL DESIGN OPTIMIZATION CONSIDERING FIDELITY UNCERTAINTIES
}

Doctorate of Applied Science, 2010

\author{
Daniel Neufeld
}

Aerospace Engineering, Ryerson University

\begin{abstract}
Aircraft conceptual design traditionally utilizes simplified analysis methods and empirical equations to establish the basic layout of new aircraft. Applying optimization methods to aircraft conceptual design may yield solutions that are found to violate constraints when more sophisticated analysis methods are introduced. The designer's confidence that proposed conceptual designs will meet their performance targets is limited when conventional optimization approaches are utilized. Therefore, there is a need for an optimization approach that takes into account the uncertainties that arise when traditional analysis methods are used in aircraft conceptual design optimization. This research introduces a new aircraft conceptual design optimization approach that utilizes the concept of Reliability Based Design Optimization (RBDO). RyeMDO, a framework for multi-objective, multidisciplinary RBDO was developed for this purpose. The performance and effectiveness of the RBDO-MDO approaches implemented in RyeMDO were evaluated to identify the most promising approaches for aircraft conceptual design optimization. Additionally, an approach for quantifying the errors introduced by approximate analysis methods was developed. The approach leverages available historical data to quantify the uncertainties introduced by approximate analysis methods in two engineering case studies: the conceptual design optimization of an aircraft wing box structure and the conceptual design optimization of a commercial aircraft. The case studies were solved with several of the most promising RBDO-MDO integrated approaches. The proposed approach yields more conservative solutions and estimates the risk associated with each solution, enabling designers to reduce the likelihood that conceptual aircraft designs will fail to meet objectives later in the design process.
\end{abstract}




\section{Acknowledgments}

I thank my advisor, Dr. Joon Chung for his many years of patience and advice. I also thank

Dr. Behdinan, who constantly provided valued advice and motivation to improve. I thank my father, John Neufeld for supporting me throughout my studies. 


\section{Contents}

1 Introduction 1

1.1 Aircraft Conceptual Design . . . . . . . . . . . . . . . 3

1.2 Uncertainty .......................... 4

1.2.1 Aleatory Uncertainty ............... 5

1.2.2 Epistemic Uncertainty . . . . . . . . . . . . . 5

1.3 Motivation .......................... 6

1.4 Outline of the Dissertation . . . . . . . . . . . 9

2 Methodology 11

2.1 Design Optimization . . . . . . . . . . . . . . 11

2.1.1 Multi-Disciplinary Design Optimization . . . . . . . . . . . 13

2.2 Uncertainty Modeling Methods . . . . . . . . . . . . . . . 21

2.2.1 Probabilistic Methods . . . . . . . . . . . . . . . 22

2.2.1.1 Simulation Methods . . . . . . . . . . . . . . 24

2.2.1.2 Analytical Methods ............. 25

2.2.2 Non-Probabilistic Methods . . . . . . . . . . . . . . . . . 27

2.2.3 Uncertainty in Design Optimization . . . . . . . . . . . . 28

2.2.4 Reliability Based Robust Design Optimization . . . . . . . . . . 29

2.2.5 Reliability and Possibility Based Design Optimization . . . . . . . 30

2.3 Reliability-Based Design Optimization . . . . . . . . . . . . . . 32

2.3.1 Reliability Assessment Strategies . . . . . . . . . . . . 32

2.3.1.1 The Reliability Index Approach . . . . . . . . . 33

2.3.1.2 The Performance Measure Approach . . . . . . . . . 35 
2.3.2 Reliability Based Optimization Integration Strategies . . . . . . 36

2.3.2.1 Double Loop Method . . . . . . . . . . . . . . . . 36

2.3.2.2 Sequential Method . . . . . . . . . . . . . . 38

2.3.2.3 Single Loop Method . . . . . . . . . . . . . . . . . 39

2.4 Summary $\ldots \ldots \ldots \ldots \ldots \ldots \ldots \ldots \ldots$

3 RyeMDO: A Multi-Discipline, Multi-Objective RBDO Package 43

3.1 Reliability-Based Multi-disciplinary Design Optimization Strategies . . . 48

$3.1 .1 \quad$ MDF Method . . . . . . . . . . . . . . . . . . . 48

3.1 .2 IDF Method . . . . . . . . . . . . . . . . . 50

3.1 .3 CO Method . . . . . . . . . . . . . . . . . 52

3.2 Validation and Benchmarking . . . . . . . . . . . . 54

3.2.1 Single Discipline Analytical Optimization . . . . . . . . . . 55

3.2.2 Single Discipline Truss Optimization _ . . . . . . . . . 58

3.2.3 Multi-Discipline Analytical Optimization . . . . . . . . . . 62

3.2.4 Multi-Discipline Truss Example Optimization . . . . . . . . . . . 65

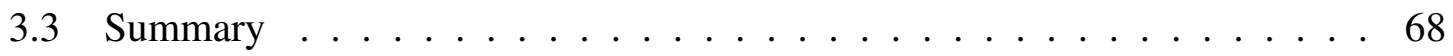

4 Aircraft Wing Box Conceptual Design Considering Model Uncertainty 71

4.1 Problem Description . . . . . . . . . . . . . . . . . . . 73

4.2 Surrogate Model . . . . . . . . . . . . . . . . . . 74

4.3 Model Error . . . . . . . . . . . . . . . . . . . . . . 75

4.4 Solution Strategy $\ldots \ldots \ldots \ldots \ldots$

4.5 Results . . . . . . . . . . . . . . . . . . . 78

4.6 Algorithm Performance Comparison . . . . . . . . . . . . . . 79

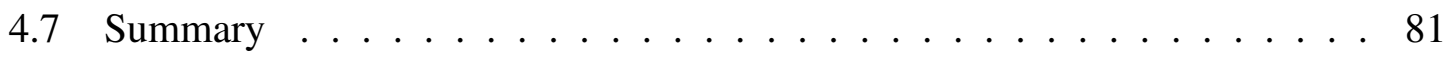

5 Aircraft Conceptual Design Considering Uncertain Contributing Analysis Methods $\quad 85$

5.1 Problem Description $\ldots \ldots \ldots \ldots \ldots \ldots$

5.2 Automated Aircraft Configuration $\ldots \ldots \ldots \ldots$

5.3 Contributing Analysis Methods . . . . . . . . . . . . . . . . . 89 
5.3.1 Aerodynamics and Stability _. . . . . . . . . . . . . . . 89

5.3.1.1 Sources of Uncertainty _ . . . . . . . . . . . . 90

5.3.2 Weight and Balance . . . . . . . . . . . . . . 92

5.3.2.1 Sources of Uncertainty . . . . . . . . . . . . . 92

5.3 .3 Performance . . . . . . . . . . . . . . . . 93

5.3.3.1 Sources of Uncertainty _ . . . . . . . . . . . . . 95

5.4 Solution Strategy ～. . . . . . . . . . . . . . . . . . . . . . 97

5.4.1 Method 1: IDF/Sequential/PMA . . . . . . . . . . . . . . 98

5.4.2 Method 2: MCS/GA . . . . . . . . . . . . . . . . . . 101

5.5 Results . . . . . . . . . . . . . . . . . . . . . . . . . . . . 104

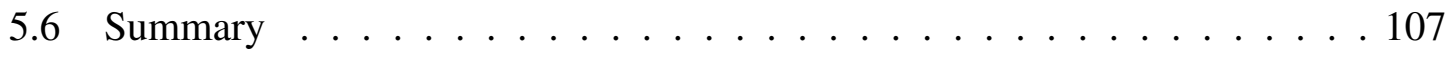

6 Conclusion 113

6.1 Future Work . . . . . . . . . . . . . . . . . . . . . 115

$\begin{array}{lr}\text { Bibliography } & 119\end{array}$

$\begin{array}{ll}\text { A Data Sources } & 137\end{array}$

B Aviation Regulations $\quad 145$ 


\section{List of Tables}

2.1 MDO Method Summary $\ldots \ldots \ldots \ldots \ldots$

3.1 Algorithm Performance Comparison . . . . . . . . . . . . . . 57

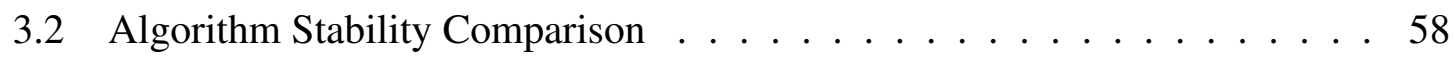

3.318 Bar Truss Design Variables … . . . . . . . . . . . . . 60

3.4 Algorithm Performance Comparison . . . . . . . . . . . . . . . 60

3.5 Algorithm Performance Comparison . . . . . . . . . . . . . . 63

3.6 Algorithm Stability Comparison _ . . . . . . . . . . . . . . 65

3.7 MDO Truss Design Variables . . . . . . . . . . . . . . . . . . . . 67

3.8 MDO Truss Example Solution $\ldots \ldots \ldots$

$4.1 \quad$ Member Attribute List . . . . . . . . . . . . . . . . . . . . 83

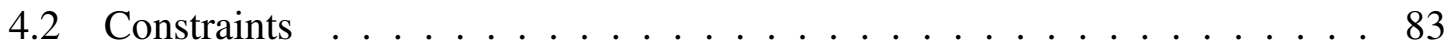

4.3 Design Variable Values - MDF/Sequential Method . . . . . . . . . . . . . 84

4.4 RBDO-MDO Performance Comparison . . . . . . . . . . . . . . . . 84

5.1 Design Goals . . . . . . . . . . . . . . . . . . . . . 87

5.2 Design and Coupling Variable List . . . . . . . . . . . . . 110

5.3 Aerodynamics and Stability Local Constraints . . . . . . . . . . . 110

5.4 Performance Local Constraints . . . . . . . . . . . . . . . . . 111

A.1 Aircraft Specification Database . . . . . . . . . . . . . 138

A.2 Engine Performance Database . . . . . . . . . . . . . . . . 139

A.3 Wing Box Database . . . . . . . . . . . . . . . 140

A.4 Wing Box Database (cont) $\ldots \ldots \ldots \ldots \ldots \ldots \ldots \ldots$ 
A.5 Wing Box Database (cont) . . . . . . . . . . . . . . 142

A.6 Wing Box Database (cont) . . . . . . . . . . . . . 143

B.1 Regulations for Fuselage Sizing . . . . . . . . . . . . . . . 145

B.2 Regulations for Fuselage Sizing (continued) . . . . . . . . . . . 146

B.3 Regulations for Fuselage Sizing (continued) . . . . . . . . . . . . . 147 


\section{List of Figures}

1.1.1 Design Process (Raymer, 1999) . . . . . . . . . . . . . . . . 3

2.1.1 Coupled System Example (Kodiyalam, 2001) … . . . . . . . . . . 14

2.1.2 MDF Method (Perez, 2004) ‥ . . . . . . . . . . . . . . 15

2.1.3 IDF Method (Perez, 2004) … . . . . . . . . . . . . . . . . 17

2.1.4 CSSO Method (Perez, 2004) ～. . . . . . . . . . . . . . . . . . . 18

2.1.5 CO Method (Perez, 2004) … . . . . . . . . . . . . . . . . . 19

2.1.6 BLISS Method . . . . . . . . . . . . . . . . 20

2.2.1 Monte-Carlo Simulation Approach . . . . . . . . . . . . . . . . 24

2.2.2 First Order Reliability Method . . . . . . . . . . . . . . . 26

2.2.3 Interval and Fuzzy Models . . . . . . . . . . . . . . . . . . 28

2.2.4 Robust Design . . . . . . . . . . . . . . . . . . . . . . . . . 30

2.2.5 Reliability Based Design Optimization . . . . . . . . . . . . . . . 31

2.3.1 RIA Approach $($ Deb, 2007) $\ldots \ldots \ldots$. . . . . . . . . . . 34

2.3.2 PMA Approach $(\mathrm{Deb}, 2007) \ldots \ldots \ldots$

2.3.3 Double Loop Method (Shan, 2008) … . . . . . . . . . . . . . 37

2.3.4 Sequential Method (Du, 2004) … . . . . . . . . . . . . . . . . 39

2.3.5 Single Loop Method (Liang, 2008) . . . . . . . . . . . . . . . . 42

3.0 .1 RyeMDO Modules . . . . . . . . . . . . . . . . . . . . 45

3.0.2 RyeMDO - Solution Procedure . . . . . . . . . . . . . . . 47

3.2.1 Single Discipline Example . . . . . . . . . . . . . . . . 56

3.2 .218 Bar Truss . . . . . . . . . . . . . . . . . . . . . . 59

3.2.3 18 Bar Truss Solution . . . . . . . . . . . . . . . . . . . 61 
3.2.4 Solution Distribution Box Plot . . . . . . . . . . . . . . . . 64

3.2.5 Decoupled Structure . . . . . . . . . . . . . . . . . . 66

3.2 .6 Optimized Truss . . . . . . . . . . . . . . . . . . . . . 67

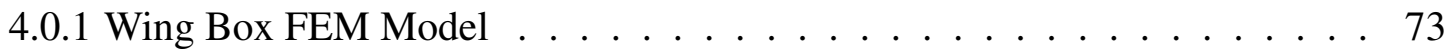

4.1.1 Structural Discipline Variables . . . . . . . . . . . . . . . . . . 74

4.3.1 Kriging Model Error Distribution: $\mu=1.0039, \sigma=0.0736 \ldots \ldots \ldots$

4.4.1 Collaborative Optimization with RBDO _ . . . . . . . . . . . . 77

4.5.1 Wing Box RBDO Results . . . . . . . . . . . . . . . . . . . . . 79

4.5 .2 Wing Planform $\ldots \ldots \ldots \ldots \ldots$

5.2.1 Aircraft Layout Example . . . . . . . . . . . . . . . . . . . . 88

5.3.1 Aerodynamics and Stability Coupling _ . . . . . . . . . . . 90

5.3.2 Weight and Balance Coupling . . . . . . . . . . . . . . . . . 92

5.3.3 Mass Error Distribution Estimates . . . . . . . . . . . . . . . . . 93

5.3.4 Flight Profile . . . . . . . . . . . . . . . . . . . . . . . . 94

5.3.5 Performance Coupling . . . . . . . . . . . . . . . . . 95

5.3.6 Propulsion Error Distribution Estimates ～. . . . . . . . . . . . . . . 97

5.4.1 Block Diagram - Method 1 . . . . . . . . . . . . . . . . . . 100

5.4.2 Block Diagram - Method 2 . . . . . . . . . . . . . . . . . . 104

5.5 .1 Fuel vs. Reliability Index . . . . . . . . . . . . . . . . . . . 105

5.5.2 Uncertain Constraints vs. Reliability Index . . . . . . . . . . . . . . 106

5.5.3 Aircraft Specifications vs. Reliability Index ～. . . . . . . . . . . . 108

5.5.4 Results Compared With the Boeing 737-800 . . . . . . . . . . . . . 109 


\title{
Nomenclature
}

\author{
Abbreviations \\ BLISS Bi-Level Integrated System Synthesis \\ CO Collaborative Optimization \\ COV Coefficient of Variation \\ CSSO Concurrent Subspace Optimization \\ DOE Design of Experiments \\ FEM Finite Element Method \\ FORM First Order Reliability Method \\ GA Genetic Algorithm \\ IDF Individual Discipline Feasible \\ ISA International Standard Atmosphere \\ MCS Monte Carlo Simulation \\ MDA Multi-Discipline Analysis \\ MDF Multi-Discipline Feasible \\ MDO Multi-Disciplinary Design Optimization \\ MPP Most Probable Point \\ PBDO Possibility Based Design Optimization \\ PDF Probability Density Function \\ PMA Performance Measure Approach
}


RBDO Reliability Based Design Optimization

RBRDO Reliability Based Robust Design Optimization

RDO Robust Design Optimization

RIA Reliability Index Approach

RSA Response Surface Approximation

SFC Specific Fuel Consumption

SFC Specific fuel consumption

SLSV Single Loop Single Variable

SQP Sequential Quadratic Programming

\section{Symbols}

$\begin{array}{ll}\varepsilon_{M_{e}} & \text { Empty mass error ratio } \\ \varepsilon_{S F C} & \text { Specific fuel consumption error ratio } \\ \varepsilon_{T_{\text {avail }}} & \text { Available thrust error ratio } \\ \varepsilon_{T_{\text {req }}} & \text { Required thrust error ratio } \\ \alpha & \text { Interval function width } \\ \beta & \text { Reliability index } \\ \varepsilon_{\sigma} & \text { Stress error ratio } \\ \Gamma_{w} & \text { Wing dihedral angle } \\ \Lambda_{h} & \text { Horizontal tail aspect ratio } \\ \lambda_{h} & \text { Horizontal tail taper ratio } \\ \lambda_{v} & \text { Vertical tail taper ratio } \\ \Lambda_{w} & \text { Wing sweep angle } \\ \lambda_{w} & \text { Wing taper ratio } \\ \mu & \text { Mean } \\ \sigma & \text { Standard deviation }\end{array}$


$\sigma_{b} \quad$ Buckling stress

$\sigma_{p} \quad$ Predicted stress

$\sigma_{F E M}$ Stress from FEM solver

$\sigma_{\max } \quad$ Ultimate stress

$\theta \quad$ PDF parameters

$A R_{v} \quad$ Vertical tail aspect ratio

$A R_{w} \quad$ Wing aspect ratio

$C_{D, 0} \quad$ Parasite drag coefficient

$C_{L, \max }$ Maximum lift coefficient

$d \quad$ Deterministic design variable vector

E Elastic modulus

$f \quad$ Objective function

$g \quad$ Constraint function

$h_{c r} \quad$ Cruise altitude

$I_{x} \quad$ Moment of inertia about x

$I_{y} \quad$ Moment of inertia about y

$I_{z} \quad$ Moment of inertia about $\mathrm{z}$

$K \quad$ Buckling coefficient

$k \quad$ Induced drag constant

$L / D \quad$ Lift to Drag ratio

$M_{e} \quad$ Aircraft empty mass

$M_{f} \quad$ Fuel mass

$M_{g} \quad$ Aircraft gross mass

$M_{c r} \quad$ Cruise Mach number

$M_{p l} \quad$ Payload mass 


\begin{tabular}{|c|c|}
\hline$N_{\text {fail }}$ & Number of failed designs in an MCS run \\
\hline$n_{\text {fuse }}$ & Fuselage configuration number \\
\hline$N_{\text {pass }}$ & Number of feasible designs in an MCS run \\
\hline$N_{\text {pax }}$ & Number of passengers \\
\hline$p$ & Uncertain parameters \\
\hline$P_{f}$ & Probability of failure \\
\hline$P_{\text {goal }}$ & Target probability of feasibility \\
\hline$R$ & Range \\
\hline$S_{h}$ & Horizontal tail area \\
\hline$S_{v}$ & Vertical tail aspect ratio \\
\hline$S_{w}$ & Wing area \\
\hline$S_{T O}$ & Takeoff distance \\
\hline$T_{s l}$ & Sea level thrust \\
\hline$U$ & Normalized uncertain variable vector \\
\hline$V_{a}$ & Approach speed \\
\hline$V_{s}$ & Stall speed \\
\hline$x$ & Local variable vector \\
\hline$x_{c g}$ & Longitudinal center of gravity \\
\hline$y$ & Coupling variable vector \\
\hline$y^{\prime}$ & Estimated coupling variable vector \\
\hline$z$ & Global variable vector \\
\hline
\end{tabular}




\section{Chapter 1}

\section{Introduction}

The aviation industry, whether military, civil, or general, is highly competitive. Customers drive demand for aircraft to be as inexpensive as possible to purchase and operate while remaining safe, reliable, and efficient. Additionally, the regulations for safety, particularly in commercial aviation, are comprehensive and demanding. Improvements in environmental performance and fuel efficiency is increasingly driving design [1]. Modern aircraft are extremely complex systems, strongly influenced by structural analysis, aerodynamic analysis, propulsion systems, avionics, and other disciplines [2]. The design of new commercial aircraft constitutes a massive investment over long development periods. Reducing the development time and cost by modernizing the design process is crucial in aircraft design [3]. Simulation tools such as the Finite Element Method (FEM) and Computational Fluid Dynamics (CFD) have been extensively studied and applied in aircraft design, particularly in the preliminary and detail design phases $[4,5,6,7]$. However, conceptual design still widely relies on engineering knowledge, historical data, and low fidelity analysis methods. Design optimization methodology has been widely applied to all phases of aircraft design, but the results from optimization processes are only as good as the contributing analysis 
methods they are based upon. Discretization, simplified analysis, and statistical or empirical methods introduce errors that propagate through the design optimization process. This can lead to problems when the design is subjected to better analysis or physical testing. It may be found that designs optimized with simplified analysis methods fail to meet performance targets later in the design process, where high fidelity analysis is employed [8]. It is important, therefore, to manage error early in the design process to decrease the likelihood of redesign while minimizing the necessary compromises to the efficiency and competitiveness of the design. Commercial aircraft designers typically leverage past experience and the large quantities of widely available data covering the dimensions, specifications, and performance of existing commercial aircraft designs $[9,10,11,12]$.

Multi-disciplinary Design Optimization (MDO) has provided methodology that can enhance the speed of the aircraft conceptual design process, rapidly identifying the optimum design based on the simplified analysis methods typically used at the conceptual level of design $[13,14,15,16,17]$. However, MDO is deterministic, and does not take into account uncertainties that can arise from various sources including approximate analysis methods.

Reliability Based Design Optimization (RBDO) is a framework for considering probabilistic variables and parameters and provides an approach to account for sources of uncertainty in design optimization [18]. The main goals of this research were to identify robust and efficient RBDO methods for multi-disciplinary design and to develop an optimization framework for aircraft conceptual design that accounts for the uncertainties that inevitably arise when approximate analysis methods are implemented in aircraft conceptual design by using historical data. 


\subsection{Aircraft Conceptual Design}

Conceptual design refers to the first phase in the design process. Figure 1.1.1 outlines the 3 phases of engineering design [11]. Conceptual design begins after establishing the design requirements. In commercial aviation, these design requirements are developed by carrying out market research and checking competing aircraft to determine the size and performance targets a new aircraft should meet to be competitive and profitable.

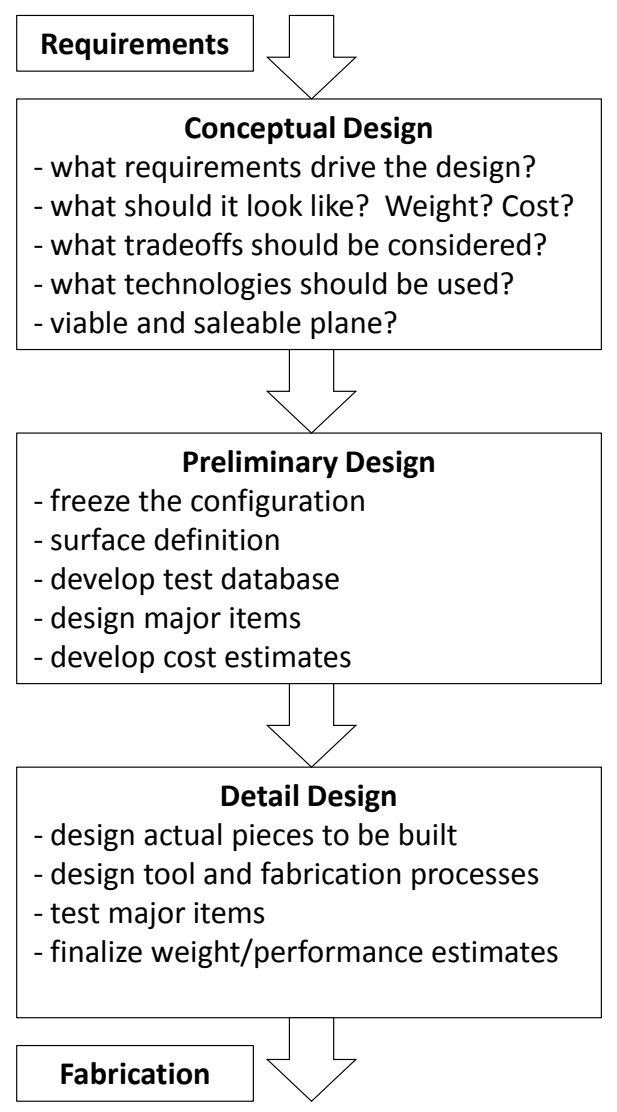

Figure 1.1.1: Design Process (Raymer, 1999)

Given a set of design requirements, the designers begin to develop the basic layout of the new aircraft, perhaps considering several alternative concepts. Designers determine whether the design requirements are reasonable - whether it is even possible to develop an aircraft that is capable of meeting the requirements. If so, the designers proceed to estimate 
the wing size, thrust requirements, fuel capacity, and other critical attributes of the aircraft. Typically, this is done with the aid of empirical equations and low fidelity analysis methods since large design changes are often frequent in conceptual design. Consequentially, the development effort and computational time required to develop physical simulations becomes unreasonable at the conceptual design phase.

Conceptual design is an iterative process where concepts are continuously refined and updated as new and more refined analysis is employed. This can be a lengthy process that requires the close collaboration of designers from several disciplines to establish an optimum trade off. For example, a design optimized strictly from an aerodynamics standpoint may lead to a highly sub-optimal design when structural analysis is carried out. Analysis disciplines are usually co-dependent, meaning an optimum aircraft will be a design with the right compromises between competing disciplines. This has led to the implementation of MDO to enhance speed and accuracy of aircraft conceptual design.

\subsection{Uncertainty}

Mathematical modeling of physical systems and engineering analysis methods is rarely deterministic. Aside from examples such as simple Newtonian dynamics which, under the right conditions, are considered exact for any practical purpose; numerical methods for calculating the response of physical systems contain sources of uncertainty. Additionally, physical systems are often affected by apparently random factors such as environmental conditions and human behavior. Sources of uncertainty usually fall into two distinct categories: aleatory uncertainty and epistemic uncertainty $[19,20]$. 


\subsubsection{Aleatory Uncertainty}

Aleatory uncertainty refers to irreducible and unpredictable variability about the behavior of a studied system [21]. This can include physical uncertainties such as the yield strength of imperfect materials, variability in the environmental loads a structure is expected to encounter, manufacturing flaws, and others [22]. Aleatory uncertainty essentially refers to all sources of uncertainty that exhibit apparent randomness and are therefore often described by probability distribution functions using gathered data.

Consider the design of an aircraft intended to fly a certain distance using minimum fuel. Typically, simulations for calculating the performance of aircraft considered in conceptual design assumes standard atmosphere properties using models such as the International Standard Atmosphere (ISA). However, in reality, the aircraft may encounter different atmospheric densities, temperatures, and wind conditions on any given day. This introduces uncertainty in the range estimates calculated with ISA properties. Since the designers have no control over the environment, the error cannot be reduced. However, the likelihood of encountering certain atmospheric conditions can be quantified using probability theory, interval analysis, or other methods by consulting experts or from a database of observations. Other commonly considered examples of Aleatory Uncertainty include flaws in structural members and manufacturing tolerance, leading to uncertainties in material strength [23].

\subsubsection{Epistemic Uncertainty}

Epistemic uncertainty is introduced when analysis methods are used that do not perfectly correspond to the physical phenomenon they are meant to describe $[21,24,25]$. Physical simulations of complex systems usually require the linearization of the governing equations. Some terms are neglected and small, high order terms are ignored to simplify the 
analysis. Additionally, translating equations into a computational simulation package introduces further approximations by implementing discretization and time stepping. Some other sources include round-off errors and the convergence tolerance of numerical methods. Epistemic uncertainty is reducible since neglected terms can be restored and time steps, grid spacing, and convergence tolerances can be reduced. Epistemic uncertainty can be assessed by introducing error terms that can be described probabilistically, by fuzzy set theory and other methods by testing the analysis methods against historical data, by comparison with higher fidelity methods, or by physical testing [25, 26].

\subsection{Motivation}

Aircraft conceptual design requires the simultaneous consideration of aerodynamics, structures, aircraft performance, flight dynamics, and many other discipline analyses. The conceptual design phase is aimed at establishing basic aircraft sizing, layout, and power requirements over a large design space. Solving conceptual design optimization problems using high fidelity approaches requires many evaluations of computationally expensive algorithms and the automated reconfiguration of the analysis models as the design changes throughout the optimization. Typically, the optimization is carried out on surrogate models such as response surface or Kriging models that are generated from a sample of results obtained from high fidelity analysis runs $[27,28,29]$. When high fidelity analysis is incorporated early in the design process, some minimum of preliminary low fidelity analysis has to be performed to narrow the scope of the optimization problem. For this reason, traditional conceptual analysis approaches are still widely used as the starting point for designing new aircraft. These methods rely heavily on empirical equations based on historical data. Statistical methods are employed for sizing engines, estimating parasite drag, and for 
predicting the final structural weight of the conceptual design. The implementation of these approximate analysis methods introduces uncertainty. Designs optimized using traditional analysis may fail to meet performance objectives in later stages of design, where high fidelity analysis methods are introduced. This can lead to costly and time consuming design revisions. Therefore, a method is needed for quantifying the expected error associated with empirical analysis in aircraft conceptual design optimization to yield designs that can be carried forward in the design process with increased confidence.

Aircraft design under uncertainty has been the subject of some recent study. Ahn et al. (2006) introduced a BLISS based RBDO framework using a simplified supersonic transport conceptual design problem [30,31]. The study assumed normal distributions with coefficients of variation of 0.3 (the ratio of the mean to standard deviation) on each of the 10 design variables considered such as wing area, span, and others describing aircraft geometry. No attempt was made to quantify the actual error distributions related to the analysis methods, design variables or the accuracy of the implemented analysis methods. Smith et al. (2003) solved a spacecraft conceptual optimization problem using RBDO to consider uncertain design variables to reflect the possibility of minor design changes later in the design process [32]. Probabilistic error terms were added to the responses of the aerodynamics and structural analysis output with assumed values of $10 \%$. The optimization problems were solved with several MDO architectures and FORM based reliability analysis methods. The aforementioned studies consider uncertainties in the design variables or parameters such as atmospheric conditions or material properties. However, the need to characterize and consider the uncertainties associated with approximate analysis methods in aircraft conceptual design has not been addressed. Furthermore, no study is currently available that provides information regarding the speed, efficiency, reliability, and accuracy of integrated RBDO and MDO strategies. 
This research proposes a new approach for commercial aircraft conceptual design optimization by improving the designer's confidence in the availability of viable results when approximate analysis methods are used. RyeMDO, a software package consisting of modules for reliability assessment, optimization approaches, and MDO methods was developed. Several methods for integrating RBDO and MDO strategies were compared by solving analytical and truss optimization case studies using RyeMDO. The speed, accuracy, and reliability of each approach were benchmarked. The most promising methods for aircraft conceptual design optimization were identified. The error associated with uncertain analysis were handled by introducing error parameters in the optimization formulation of two engineering case studies: a wing box conceptual optimization case study and an aircraft conceptual design optimization case study. The characteristics of the errors were evaluated by comparing the results of the approximate analysis methods with a database of high fidelity results for the wing box optimization case study and a specification database of currently available aircraft designs for the aircraft conceptual design optimization case. The results indicate that when traditional deterministic optimization methods are used, designs are located at or near at least one constraint boundary, and may be prone to failure when validated by physical testing or when high fidelity analysis is used later in the design process. Implementing RBDO produced more conservative designs, moving designs away from active constraint boundaries. The designers may rely on the optimum solutions with increased confidence relative to deterministic approaches when uncertain analysis methods are used. 


\subsection{Outline of the Dissertation}

Chapter 2 reviews design optimization and uncertainty analysis methods. Section 2.1 reviews single and multi-discipline deterministic design optimization. Section 2.2 describes some alternative methods for quantifying uncertainty and the methods used to assess uncertainty within design optimization frameworks. Section 2.3 describes some of the alternative methods for reliability based design optimization. Different strategies for reliability assessment are reviewed as well as the common integration strategies for incorporating reliability analysis in design optimizations. Chapter 3 describes the development of RyeMDO and its usage. Additionally, the implemented algorithms were validated and benchmarked. The efficiency and reliability of each approach were evaluated using two optimization problems: an analytical problem and a truss optimization problem for both single-discipline and multidiscipline optimizations. Chapter 4 implements the most promising methods by solving a practical, multi-discipline engineering case study: an aircraft wing box optimization using an approximate analysis method in the form of a surrogate model. Chapter 5 presents an approach for the design optimization of a commercial aircraft conceptual design that ac-

counts for the uncertainties introduced by traditional conceptual design methodology. This is followed by some conclusions and an overview of future research in Chapter 6. 


\section{Chapter 2}

\section{Methodology}

This chapter reviews the concept of design optimization and the strategies for handling sources of uncertainty in design optimization. Section 2.1 reviews the concept of MDO. Section 2.2 reviews methods for modeling uncertainty in design optimization. Section 2.3 reviews the concept and methodologies of RBDO.

\subsection{Design Optimization}

Design optimization refers to computational methods used to search for designs that are as efficient and effective as possible. The mathematical statement of design optimization problems takes the form of an objective function that calculates a value that represents the critical measure of design performance or merit. The optimum design is the design that is found to have a minimum merit function while satisfying all constraints. Constraints are formulated as statements of equality or inequality that must be satisfied to keep the design feasible. Additionally, search boundaries are usually specified. A typical design optimization problem statement is given in equation 2.1.1 where the goal of the optimizer 
is to search for the deterministic design variable vector $d$ that minimizes the merit or objective function, $f$, while satisfying any constraint equality or inequality functions, $g_{i}$. The objective function, $f$, is a function of the deterministic design variable vector, $d$, the output of any contributing analysis tools, $y$, and constant parameters, $p$.

$$
\begin{gathered}
\min f(d, p, y(d, p)) \\
\text { s.t. } g_{i}(d, p, y(d, p)) \leq 0 \text { where } i=1, \ldots, N_{\text {cons }} \\
d_{l} \leq d \leq d_{u}
\end{gathered}
$$

There are many well known optimization algorithms currently available. The algorithms implemented in this research are briefly reviewed as follows. Both deterministic and stochastic algorithms were implemented including the well-known Sequential Quadratic Programming (SQP) algorithm and a multi-objective Genetic Algorithm (GA). SQP implements gradient information about a starting point to determine the direction of steepest slope [33, 34]. Local quadratic approximation functions for the objective and constraint functions are developed and solved in a sequence of 1 dimensional optimizations similar to the classic Newton's Method. The process repeats recursively until the optimality criteria are satisfied. The gradients of the objective and constraint functions are typically calculated using finite-differencing if they cannot be defined analytically. As a consequence, SQP requires the objective and constraint functions to be sufficiently smooth for accurate calculation of the gradient information. Gradient based methods find the nearest local maximum or minimum of an optimization problem. There is no guarantee that better solutions are not to be found elsewhere in the solution space [35]. SQP is one of the most successful and widely implemented algorithms for solving optimization problems with non-linear constraints [33]. GAs are stochastic methods that mimic the concept of natural selection on a population of randomly generated designs $[36,37]$. There are many variations in the de- 
sign of GAs, but most implement the same concepts: a mutation component that randomly alters design variables in the population and a crossover component that combines the better designs to produce offspring. The designer defines a fitness function that gives advantage to designs that are feasible and exhibit good objective function performance. The GA implements a selection scheme that ranks the designs and assigns crossover probabilities proportionally to the fitness of the individuals in the population, producing a new generation of designs. GAs generally require more function evaluations than gradient based approaches, but are capable of handling non-smooth objective and constraint functions more effectively. Since GAs are population based, it is possible to simultaneously consider more than one objective function. Multi-objective optimization with gradient algorithms requires solving multiple full optimizations. The GA implemented in this research is described in Langer $[38,39,40]$.

Multi-objective optimization simultaneously optimizes two or more conflicting objective functions. Unlike single-objective optimization, a set of results are obtained rather than a single solution. The results form a trade-off curve between each objective. Each solution on the curve is referred to as a Pareto-optimal solution. A Pareto-optimal solutions are defined as solutions where improvements in one objective function are only possible by regressions in at least one other objective function.

\subsubsection{Multi-Disciplinary Design Optimization}

MDO can be defined as "a methodology for the design of systems in which strong interaction between disciplines motivates designers to simultaneously manipulate variables in several disciplines [16]." Independent optimizations of individual disciplines considering local goals does not guarantee an optimum overall design, which requires the consideration of the synergy between each contributing analysis method [41]. Modern engineering opti- 
mization has reached a level of complexity that nearly always requires a strategy to handle many coupled disciplines [16, 42, 43]. Inter-disciplinary coupling occurs when the output of one analysis package is required as input for another independent analysis package. This creates a more complex computational problem than single-discipline optimization. Aerospace conceptual design presents a classic example of a coupled system. Figure 2.1.1, adapted from Kodiyalam et al. shows the interaction between disciplines for a hypothetical aircraft conceptual design process [41]. System design variables are shared by all disciplines and denoted by $Z$. Local variables, $X$, are specific to individual disciplines and $Y$ denotes the information pathway from one discipline to another. The aerodynamics solver supplies the drag properties that the performance analysis needs in order to run. In turn, the performance analysis supplies the Mach number that the aerodynamics discipline needs to compute the aircraft drag. Similar couplings are indicated between the other disciplines as well.

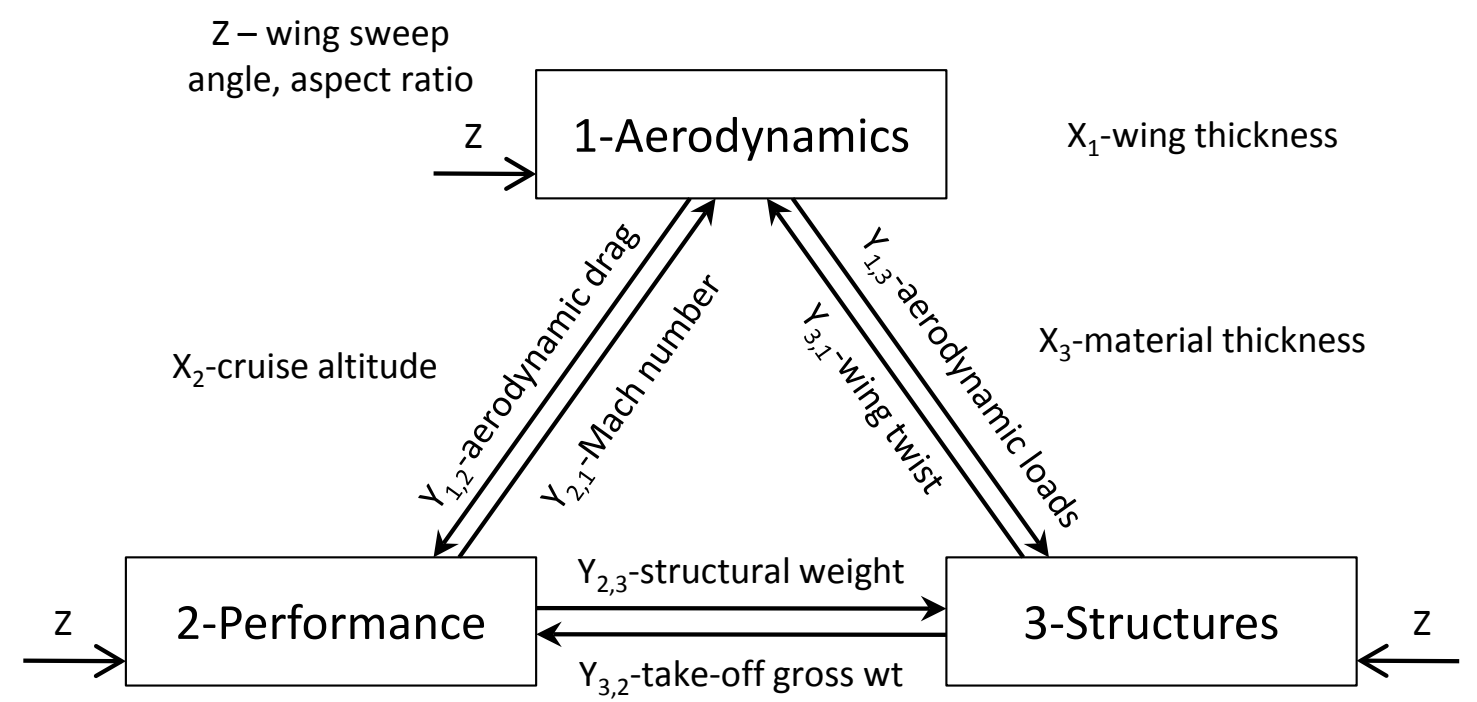

Figure 2.1.1: Coupled System Example (Kodiyalam, 2001)

There are many strategies for handling the optimization of coupled systems. MDO algo- 
rithms manage the design variables and constraints of each discipline while ensuring that the local design variables and discipline outputs held by each discipline are compatible at the solution point. The earliest and most commonly applied approach is the MultiDiscipline Feasible (MDF) method. MDF was a term introduced by Cramer et al. (1994) for methods that implement a system analysis to solve for compatible coupling conditions whenever any design variables are adjusted [41, 44]. A system analysis, referred to as Multi-Discipline Analysis or MDA, refers to an iterative process that solves for compatible coupling variables given an initial starting estimate. The algorithm block diagram is shown in Figure 2.1.2 where the global variables (variables required for evaluating the objective function or shared between the disciplines) are denoted by $z$, the local variables (variables that only influence one discipline) are denoted by $x$, and the coupling variables are denoted by $y$.

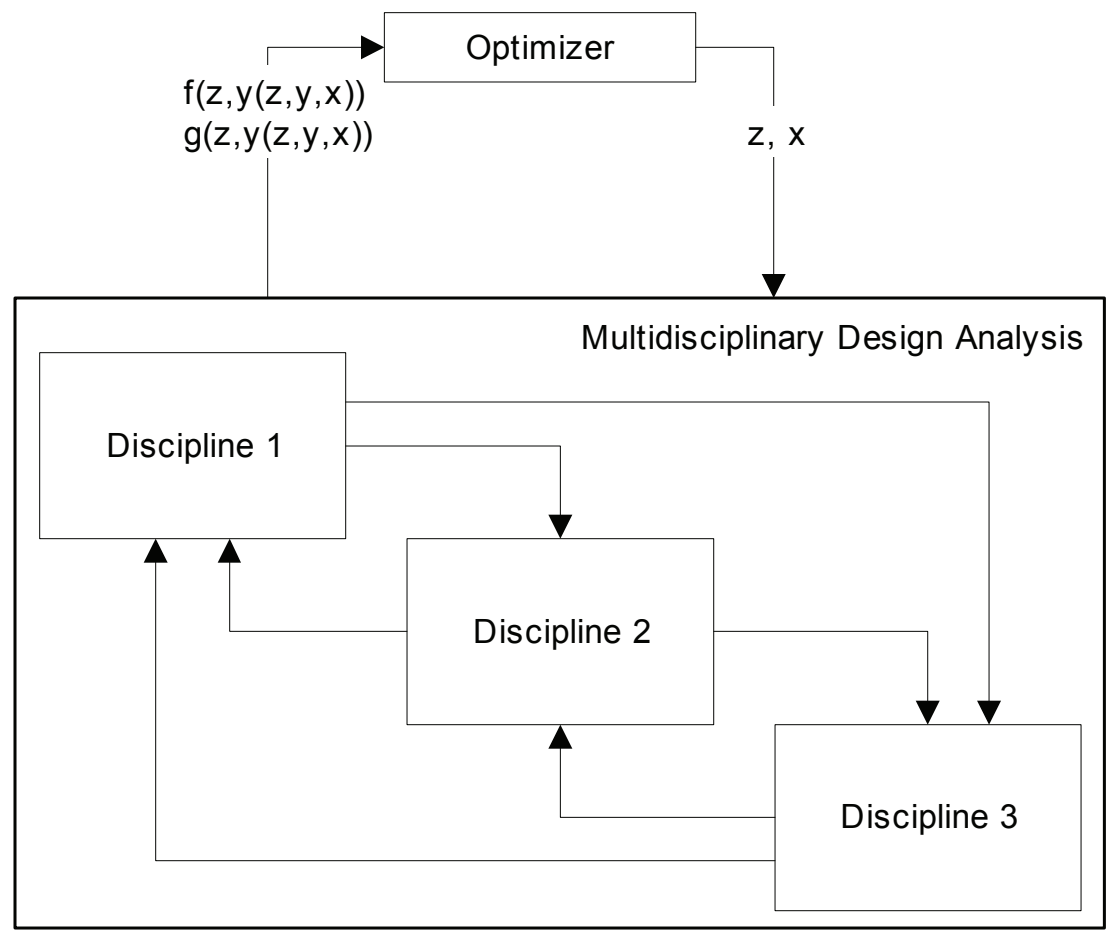

Figure 2.1.2: MDF Method (Perez, 2004) 
The MDA loop must run every time the design variables are adjusted, including for the calculation of gradients by finite differencing in gradient-based optimizers such as SQP [41, 44, 45]. Consequentially, the MDA loop must be solved with sufficient precision for the accurate calculation of gradients. This considerably increases the computational effort relative to single discipline optimization, and therefore led to more advanced methods of handling the coupling between discipline analysis including the Individual Discipline Feasible (IDF) method.

The IDF method eliminates the MDA loop and the drawbacks associated with that approach by augmenting the system design variables with coupling variable estimates. Auxiliary constraints are introduced to force the discrepancy between the discipline analysis outputs and the estimated coupling variable values to vanish by the end of the optimization. Unlike the MDF method, designs that emerge at each iteration of the optimizer may not be feasible until the optimization has converged. In other words, the coupling variables used in one discipline may not match those of another discipline until convergence. The algorithm block diagram is shown in Figure 2.1.3 where $y^{\prime}$ is the estimated coupling variable vector and $y$ is the calculated coupling variable vector.

Studies on both analytical problems and engineering problems consistently find that, with some exceptions, IDF is significantly more computationally efficient than MDF methods $[43,46,45,47,48]$. These exceptions include problems that have a very large number of coupling variables. This has the effect of greatly increasing the dimensionality of the system optimization and requires the introduction of many auxiliary compatibility constraints, which can lead to instability.

Both the MDF and IDF methods are considered Single Level methods - methods that implement only one system optimizer. A second class of MDO methods include inner optimization loops under a global or co-ordination optimization. Multi-level methods such as Col- 


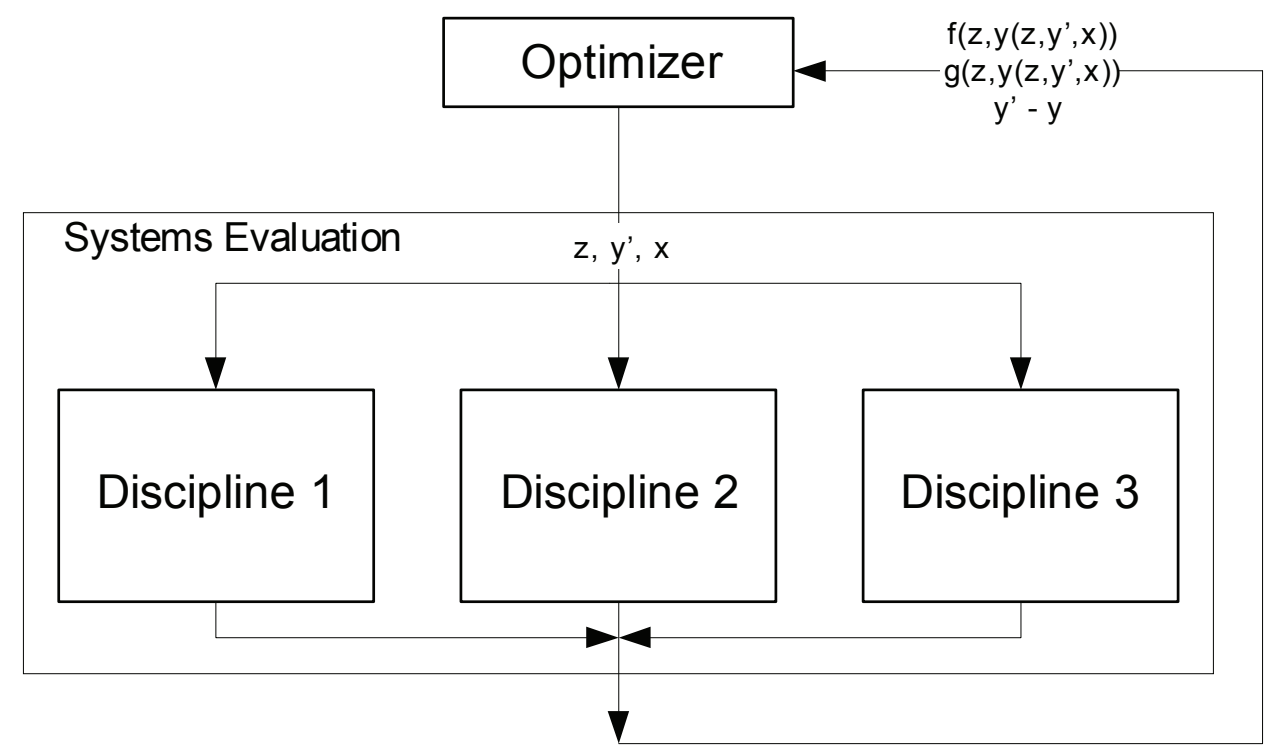

Figure 2.1.3: IDF Method (Perez, 2004)

laborative Optimization (CO), Concurrent Subspace Optimization (CSSO), and Bi-Level Integrated System Synthesis (BLISS) were developed to improve the efficiency of MDO optimizations for systems with a low coupling bandwidth, or rather, systems that have few shared design and coupling variables and many local design variables [16]. These systems can be broken down into optimization sub-problems whereby each discipline analysis (including discipline-specific constraints) interacts with its own local optimizer, leaving the system optimizer to co-ordinate inter-discipline compatibility and any shared or coupling variables.

The CSSO method, proposed by Sobieszczanski-sobieski (1988) was among the first multilevel MDO architectures to emerge $[49,50,51,52]$. CSSO mimics design strategies in which analysis groups are responsible for optimizing local components and compromises between different disciplines are made by a coordinator, as shown in Figure 2.1.4 [41, 46]. In CSSO, the analysis at local discipline levels approximates the response of the system and the other disciplines using approximations derived from the global sensitivity equations, 
which are updated at every cycle.

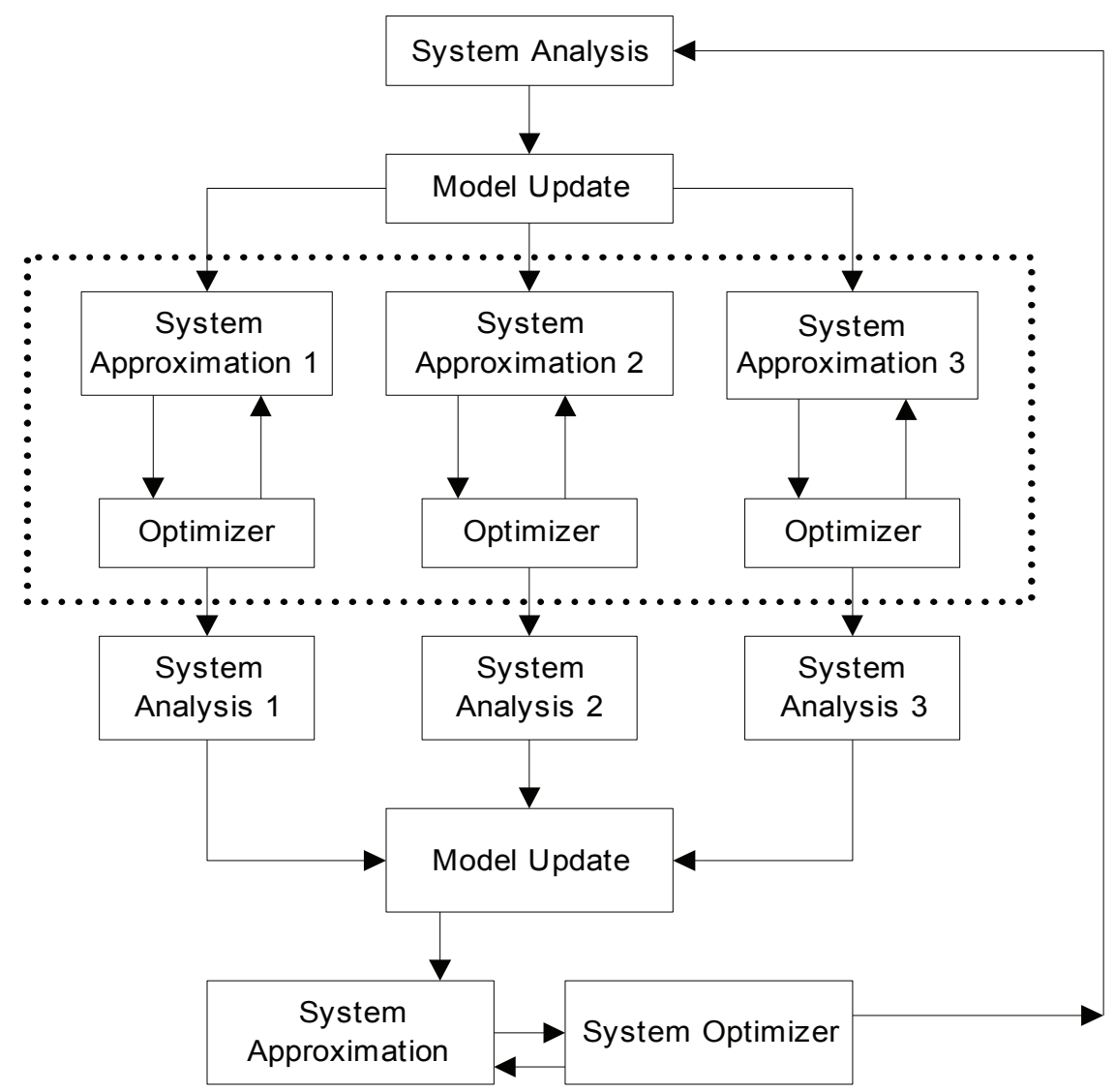

Figure 2.1.4: CSSO Method (Perez, 2004)

Introduced by Braun (1995), the CO method proposed an alternative bi-level approach [53, $54,55]$. CO decomposes the problem into one local optimization for each discipline under a global optimizer that co-ordinates discipline target values. The global optimizer handles only shared and coupling variables, leaving local discipline variables and constraints to be handled by the corresponding local optimizer. The local optimizers minimize discrepancies between the local values of the shared variables and the coupling variables to the global values. The $\mathrm{CO}$ algorithm block diagram is shown in Figure 2.1.5 [46].

The BLISS method, proposed by Sobieszczanski-Sobieski is an MDO approach that im- 


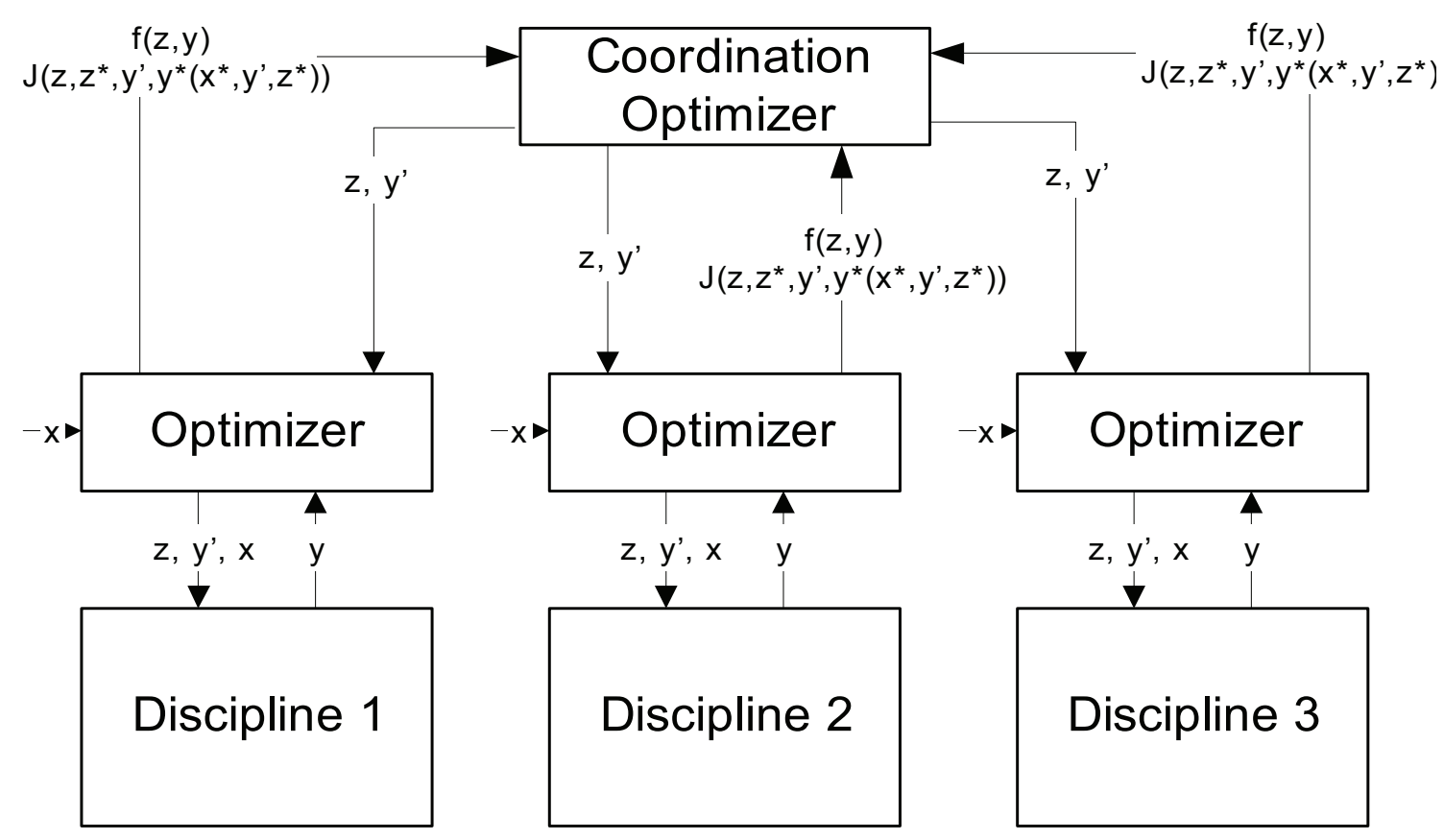

Figure 2.1.5: CO Method (Perez, 2004)

plements the global sensitivity equations to approximate the coupling effects of the local discipline optimizations on the system objective function [56]. Like CO, BLISS is a bi-level method with system and local optimizers. However, in BLISS, local optimizers only adjust local variables, leaving global variables constant and the system optimizer only adjusts system variables. The algorithm block diagram is shown in Figure 2.1.6 [46]. Improvements to the BLISS method were introduced which implement response surface approximation models to provide better estimates of discipline responses [31].

Evaluations of the performance, implementation, and robustness of multi-level MDO approaches suggest that there is a substantial increase in both computational cost and implementation effort associated with multi-level approaches [43, 46, 47, 48, 57] . However, multi-level approaches are by their nature, conducive to parallelization, where complete subsystem optimizers can be developed by different design teams. They may run on different hardware and can implement different optimizers that are particularly suitable for the 


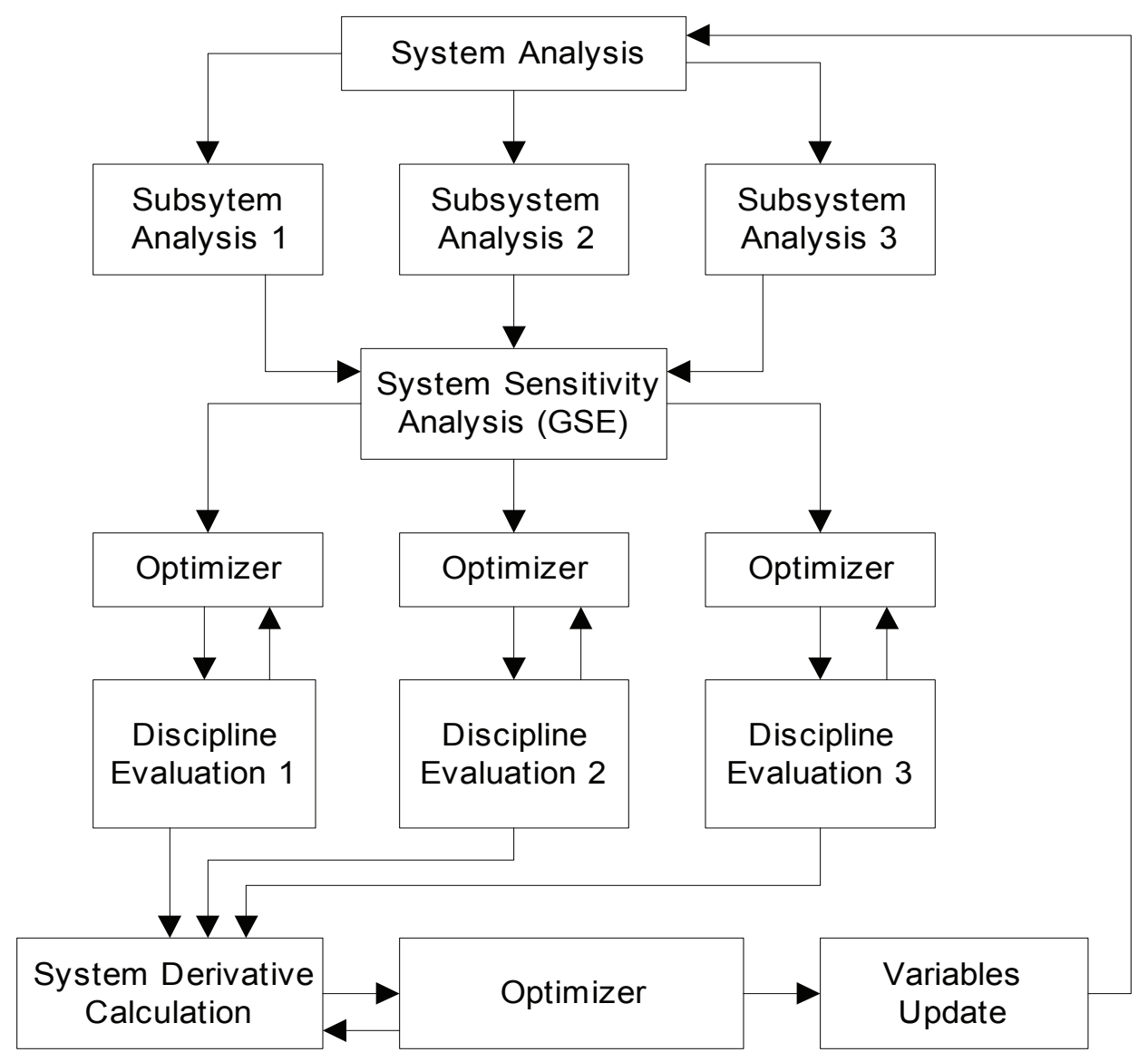

Figure 2.1.6: BLISS Method

characteristics of the local objective functions and constraints. The general properties of the MDO schemes are outlined in Table 2.1.

Aircraft design depends on the synthesis of many disciplines and has been widely studied in MDO literature. A complete MDO formulation would include disciplines such as structures, aerodynamics, performance, avionics, stability, cost, manufacturability, and so on. For practical reasons, the list is nearly always reduced when solving aerospace problems. The development of the multi-level MDO schemes including Collaborative Optimization (CO) and Bi-Level Integrated System Synthesis (BLISS) repeatedly implemented aerospace conceptual design problems as test subjects for the proposed algorithms $[54,58]$. 
Table 2.1: MDO Method Summary

\begin{tabular}{lll}
\hline Method & Advantages & Disadvantages \\
\hline MDF & -Reduced problem dimensions & -MDA required \\
& -Performance advantage for problems having many & -Convergence of MDA loop must be very precise \\
& coupling variables & \\
\hline IDF & -Simple problem formulation & \\
& -No inner loops & -Increased optimization problem dimensions \\
& -Performance advantages for problems having & -Introduction of equality compatibility constraints \\
& moderate numbers of coupling variables & \\
\hline CO & -Facilitates distributed computing & -Performance and convergence poor for problems \\
& -Enables independent optimization of disciplines & having high coupling bandwidth \\
& -Emulates human organizational structures & -Introduction of equality compatibility constraints \\
& -Efficient for problems having very large numbers of & \\
\hline CSSO & local discipline variables & \\
& -Facilitates distributed computing & -MDA required \\
& -Efficient when analytical gradients are available & -Computationally expensive calculation of sensitivity \\
& & information required \\
& & -Complex problem formulation \\
& & -Reduced accuracy \\
\hline BLISS & -Enables independent optimization of disciplines & -Computationally expensive calculation of sensitivity \\
& -Facilitates distributed computing & information required \\
& -Usually more computationally efficient than other & -Complex problem formulation \\
& multi-level methods such as CO or CSSO & \\
\hline
\end{tabular}

MDO has enabled designers to consider new discipline analyses in aircraft conceptual design. Antoine et al. (2005) introduced an environmental performance discipline for commercial aircraft conceptual design [1]. A flight control augmentation analysis was implemented in Perez et al. to improve the aerodynamic efficiency of commercial aircraft [59]. Aronstein [14] considered sonic boom analysis and Willcox [60] implemented a cost analysis discipline. Section 2.2 outlines different approaches for quantifying uncertainty and the concepts behind design optimization strategies that consider the influence of uncertainty in either the design variables, parameters, or in the contributing analysis methods.

\subsection{Uncertainty Modeling Methods}

Computational design optimization has enabled designers to explore the solution space of engineering problems with great accuracy and efficiency when compared with traditional 
conceptual design. Design optimization methods are capable of a level of precision that enables them to precisely locate the optimum solution for a given set of design equations. A consequence of this level of precision is that the optimum solution points are almost

invariably found to lie directly on one or more of the constraint boundaries [61]. Any deviation in the designer's assumptions (i.e. the material strength or the manufacturing precision of a structural member) or approximate analysis methods may result in the failure of the optimized design when it is fabricated and tested or subjected to higher fidelity analysis. Any design variable, parameter, or any output from analysis codes in a given optimization problem can be considered as uncertain quantities provided that the uncertainty can be mathematically represented. Many methods currently exist for quantifying the behavior of aleatory and epistemic uncertainty. The methods most often applied in design optimization are Interval Analysis, Fuzzy Numbers, and Probability Theory. The choice of method is driven by the quantity of information available to the designer about the source of uncertainty. In general, sources of uncertainty where there is insufficient data to estimate a probability density function (PDF) accurately, interval analysis or fuzzy numbers are preferred $[62,63,64,65]$.

\subsubsection{Probabilistic Methods}

Sources of uncertainty can be modeled using probability theory provided there is sufficient data to determine a probability distribution shape and parameters. A suitable PDF must be identified for each source of aleatory or epistemic uncertainty. This can be accomplished only if there is sufficient statistical data available. Otherwise, the designer must assume a particular PDF, perhaps based on the designer's experience, or implement one of the other strategies. In design optimization, failure of the system is characterized by constraint functions, otherwise known as limit state functions. These functions are defined as inequality 
conditions. If they are functions of any uncertain input, including uncertain variables or parameters, the output of the constraint function will also be uncertain. A probabilistic version of the optimization problem statement is shown in equation 2.2.1, where the design variables, $x$, and the parameters, $p$, are assumed to be uncertain and randomly distributed according to corresponding PDFs. The objective function is evaluated at the mean values of the uncertain terms. The constraint functions become probabilistic, where a target maximum probability of failure, $P_{f}$, is enforced. Recall that $g \leq 0$ was defined as feasible and $g>0$ as infeasible.

$$
\begin{gathered}
\min f(\bar{x}, \bar{p}, y(\bar{x}, \bar{p})) \\
\text { where } i=1, \ldots, N_{\text {cons }} \\
\text { s.t. } P\left[g_{i}(x, p, y(x, p))>0\right] \leq P_{f} \\
x_{l} \leq x \leq x_{u}
\end{gathered}
$$

Every uncertain variable and parameter may have distinct PDF functions. Therefore, the probability of failure of a limit state (constraint) is calculated by integrating a joint probability density function over the constraint boundary, $g$. The joint PDF is given in equation 2.2.3 where $f_{X_{1} \ldots X_{N}}$ is the joint PDF of a set, $X$, of $n$ random variables and $x$ represents a given realization of $X$. Exact solution of equation 2.2.3 is rarely possible [66]. Approximate methods are typically used. These methods are either simulation based or analytical simplifications.

$$
P(g>0)=P_{f}=\int_{g>0} f_{X_{1} \ldots X_{n}}\left(x_{1} \ldots x_{n}\right) d x_{1} \ldots d x_{n}
$$




\subsubsection{Simulation Methods}

Simulation approaches usually implement a Monte-Carlo Simulation (MCS) scheme [67, $68,69,70]$. MCS reliability analysis works by evaluating constraint functions with many randomly sampled design variable vectors according to the specified PDF functions for each variable. The probability of failure is approximated by the ratio of trials where $g(x)$, is infeasible to the total number of trials, as shown in Figure 2.2.1.

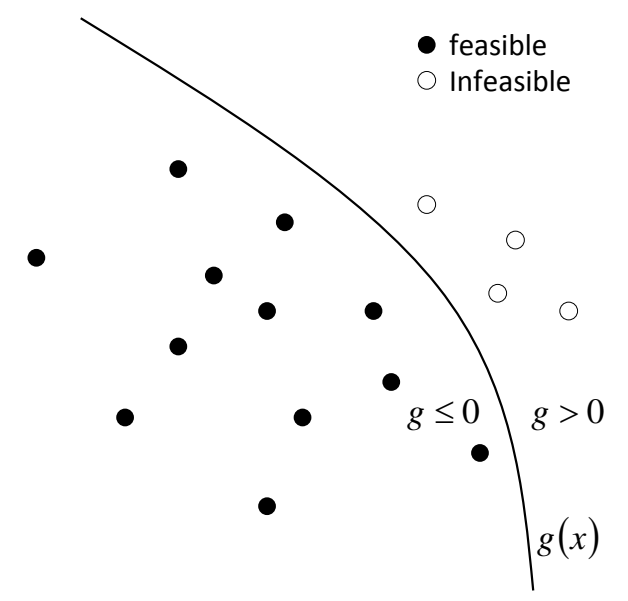

Figure 2.2.1: Monte-Carlo Simulation Approach

MCS based approaches are accurate for large sample sizes, and are usually designed to run recursively until the relative error is below a specified tolerance. However, when enforcing very small failure probabilities, the sample size must be very large to achieve any accuracy. Evaluating small failure probabilities therefore requires very large numbers of constraint function evaluations. Sample sizes are usually limited to prevent unacceptable computation times particularly when the constraint functions implement costly physics simulations. As a consequence, the predicted failure probabilities can exhibit some scatter. This is problematic when MCS is used in optimization problems using gradient-based optimizers, which rely on finite-differencing to evaluate constraint function gradients [71, 72]. However, simulation methods are still widely used for evaluating constraint functions that are 
highly non-linear with respect to the uncertain variables and parameters $[73,74,75]$. The computational cost can be mitigated by replacing the constraints with approximation models such as Response Surface Approximations (RSA) or Kriging models. The surrogates rather than the system equations are sampled to evaluate the failure probability $[76,77,78]$. RSA models are multi-dimensional regression curves. The curve is a best fit model generated from a collection of data points, and may not pass through all or any of the original points. Kriging models function like multi-dimensional splines - the model passes through every data point used to generate the curve. MCS methods have the additional advantage that all of the input constraint functions may be evaluated simultaneously. This is not the case for analytical approaches, which must evaluate each constraint function individually at different variable states.

\subsubsection{Analytical Methods}

Analytical methods solve the joint PDF by making local approximations to the constraint function boundary. The First Order Reliability Method (FORM) implements a linear approximation of the constraint function in standard space. This has been shown to be a good approximation for small failure probabilities [79]. Standard space is defined as normally distributed with a mean of $\mu=0$, and a standard deviation of $\sigma=1$. It should be noted that the random variables do not necessarily have to be normally distributed if an equation exists for transforming the random variable into standard space. This is accomplished by using Rosenblatt transformations, $U=T(X, \theta)$, where $\theta$ represents the distribution parameters (i.e. $\mu, \sigma$ for normal distributions) of $X[77,80]$. The Rosenblatt transformation for a normally distributed random variable set is $X=\mu+\sigma U$. The FORM method is illustrated in Figure 2.2.2 for a problem having two random variables [81].

The reliability index, given by $\beta$, is the number of standard deviations from the mean of the 


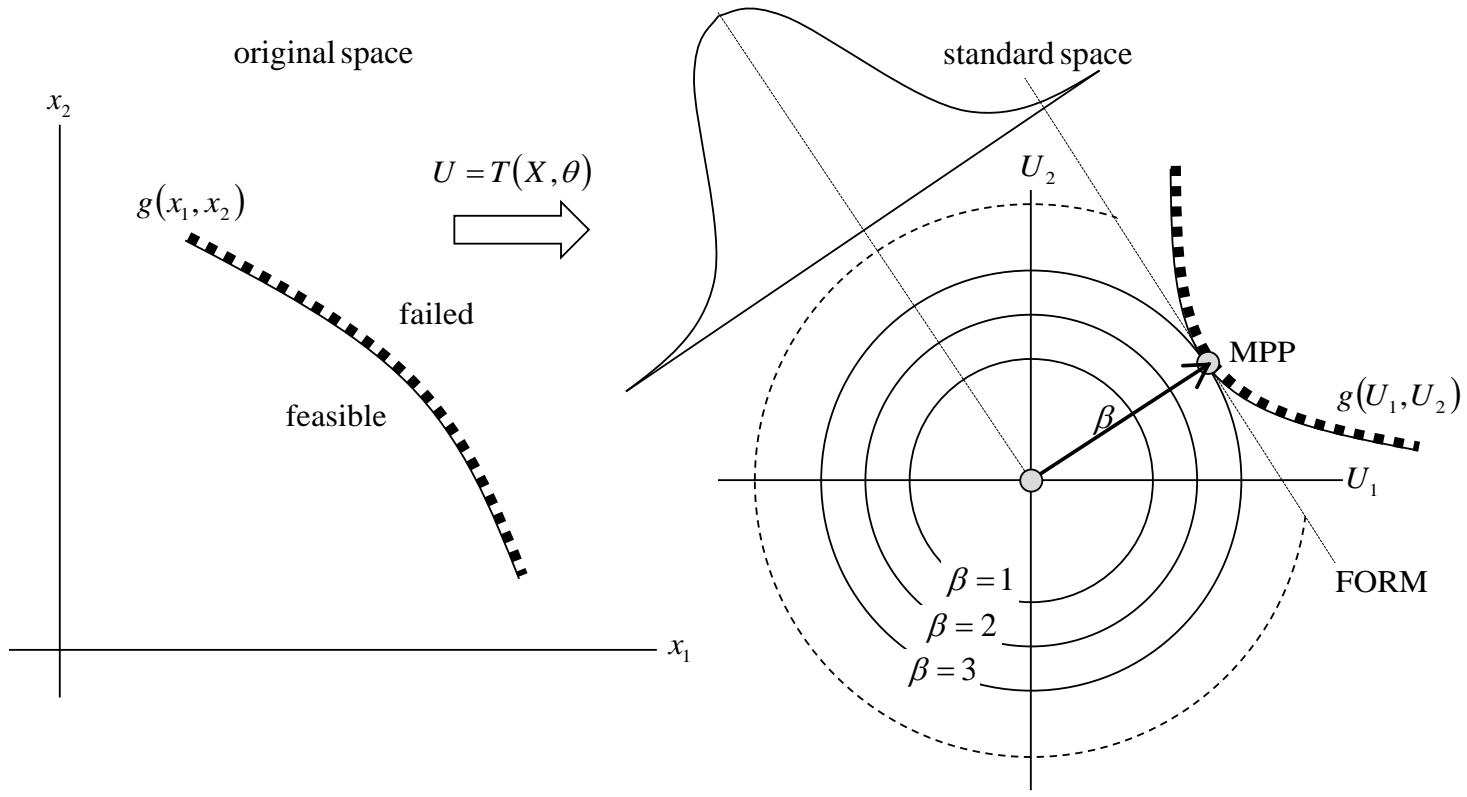

Figure 2.2.2: First Order Reliability Method

closest approach of the constraint function in standard space. The point of closest approach is referred to as the Most Probable Point (MPP). The reliability index, $\beta$, can therefore be defined as $\beta=\left\|U^{*}\right\|$ when $\mathrm{g}\left(U^{*}\right)$ is a minimum. This is usually found by minimizing $g(U)$ s.t. $\|U\|=\beta_{\text {goal }}$ where $\beta_{\text {goal }}$ is the target reliability index defined by the designer, corresponding to the maximum allowable failure probability. Methods for solving FORM are outlined in Section 2.3. Other analytical methods include higher order approximations of the constraint function, such as the Second Order Reliability Method (SORM). These methods are more costly to solve, but are more accurate for constraint functions that are highly non-linear [18]. Probabilistic methods for modeling uncertainty can only be applied when there is sufficient data available for each source of uncertainty to identify the shape and parameters of a PDF function. When there is insufficient data, a common practice for probabilistic modeling is to assume a uniform distribution between the highest and lowest observed values of a random uncertain variable $[82,83]$. However, there are several nonprobabilistic methods specifically developed for dealing with sources of uncertainty when 
knowledge or data is sparse. Several of these methods are briefly reviewed in Section 2.2.2.

\subsubsection{Non-Probabilistic Methods}

Non-probabilistic methods are used to model uncertainty when there is insufficient data to develop a good estimate of the PDF shape or parameters. Several of the most common methods are Interval and Fuzzy Modeling, Evidence Theory, and Convex Modeling. Moller et al. reviews the theories of interval analysis, fuzzy modeling, and evidence theory in [84]. Convex modeling method are reviewed in Ben-Haim et al. [85].

Interval modeling is a widely used non-probabilistic method for representing uncertainty $[86,87,88]$. It is based on the following idea. If a number $X$ is not known precisely but is known to lie between two hard boundaries $[A, B]$, any mathematical processes that are applied to $X$ can be applied to the interval $[A, B]$ to find an output interval that contains the solution. Interval analysis does not provide any indication of where the solution is likely to lie within the boundaries, only providing the boundaries themselves. The input intervals are typically estimated using expert knowledge. Experts give the best and worst case scenario for a particular uncertain variable or parameter [84]. Fuzzy numbers extend the concept of interval analysis by the addition of a membership function that describes the degree of membership an observation has within the interval, as shown in Figure 2.2.3. A triangular membership function is shown. However, any membership function shape can be used. However, some solution strategies such as FORM can only be implemented on convex membership functions $[89,90]$. Interval analysis can be considered as a special case of fuzzy modeling, where the membership function to an interval is binary, where 0 is non-membership, and 1 is complete membership. Several methods are available for handling interval and fuzzy uncertainty, including FORM, which can be extended to handle constraint functions with fuzzy numbers. The determination of an appropriate membership 
function is usually accomplished by consulting experts rather than statistical analysis. Both subjective knowledge and objective data can be used [91, 92, 93]. Fuzzy modeling is therefore referred to as possibilistic rather than probabilistic.

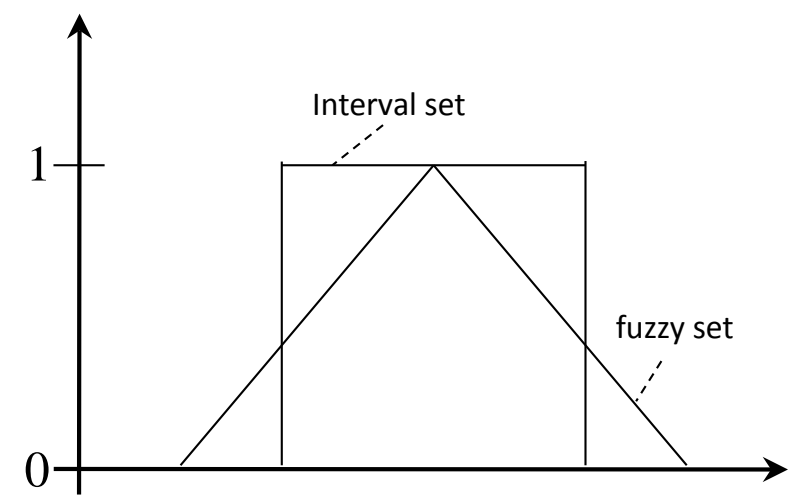

Figure 2.2.3: Interval and Fuzzy Models

\subsubsection{Uncertainty in Design Optimization}

Design optimization under uncertainty extends traditional design optimization methods by integrating uncertainty modeling to predict the influence of uncertain variables or parameters on a solution, yielding more conservative designs that account for the input uncertainty. Three disciplines have emerged for handling design with uncertainty: Reliability Based Design Optimization (RBDO), Possibility Based Design Optimization (PBDO) [94], and Reliability Based Robust Design Optimization (RBRDO) [95, 96]. Both RBDO and PBDO are optimization strategies that enforce a desired likelihood that constraints will be satisfied when the design is fabricated and tested or subjected to more reliable analysis methods. RBDO achieves this by modeling each source of error with PDF functions and determining their influence on the optimization constraints. RBDO determines an optimum design that complies with all constraints to a desired level of probability, yielding more conservative 
designs than deterministic optimization. RBDO is used when there is sufficient statistical data to make good estimates of the probability distributions for each input source of uncertainty. PBDO was developed for problems containing sources of uncertainty where insufficient knowledge or data exists to build accurate probability distribution models. Errors are estimated by establishing an interval of highest and lowest expected errors. The errors are then represented by the interval or by fuzzy membership functions. Optimization results are also expressed as intervals or fuzzy numbers. In general, PBDO methods produce more conservative optimization results than RBDO. However, problems with limited data can still be solved with RBDO by assuming a uniform probability distribution over an interval, producing more conservative designs [97]. RBRDO is concerned with minimizing the expected variance in the output of an optimization process. Section 2.3 introduces the RBDO methodology including methods for reliability assessment and some alternative approaches for integrating reliability assessment in design optimization.

\subsubsection{Reliability Based Robust Design Optimization}

Robust design is an optimization approach aimed at minimizing the sensitivity of the solution to variations in the input uncertain variables and parameters $[98,99]$. The location of the true optimum design can be located in a region where small variations in the uncertain parameter lead to very large variation in the objective or constraint function output, as shown in Figure 2.2.4.

When applied to the objective function, the method is referred to as Robust Design Optimization (RDO). The designs are usually constrained such that the output variance of the objective function is below a specified limit. The constraint functions are specified such that the boundaries of the output variance of each constraint lie within feasible design space. When the constraint function variance is considered, the method is referred 


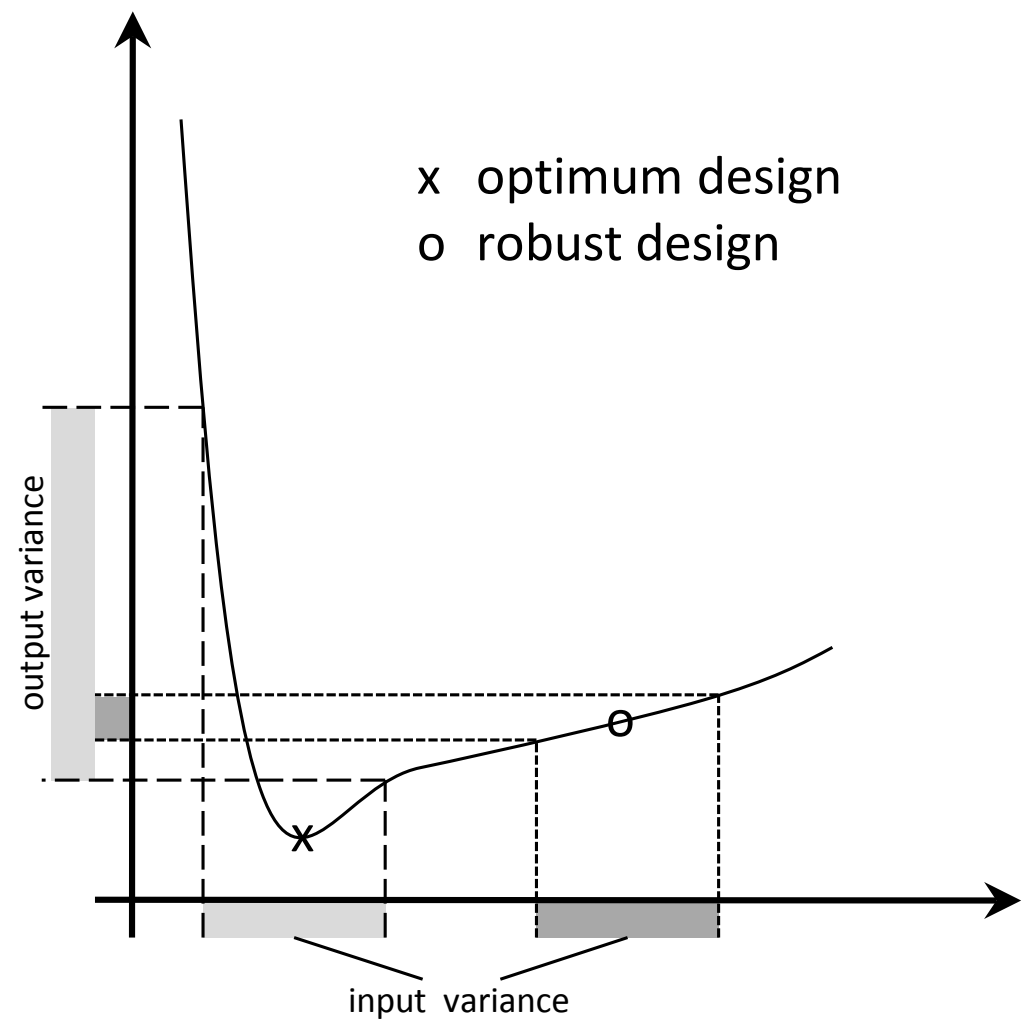

Figure 2.2.4: Robust Design

to as Reliability Based Robust Design Optimization (RBRDO). Both probability theory, interval analysis, and fuzzy sets are applicable to robust design. The input variance can be represented by fuzzy numbers, intervals, or a PDF function. Robust design optimization algorithms are based on running a series of experiments with variations in the uncertain parameters [95]. Robust design optimization approaches are described comprehensively in [100]. Probabilistic methods are not required in robust design methods.

\subsubsection{Reliability and Possibility Based Design Optimization}

RBDO is concerned with determining optimum designs that have constraint failure probabilities lower than a specified limit. PBDO searches for designs where the vertices of 
a fuzzy set lie within feasible design space, as shown in Figure 2.2.5. The symbol $\beta$ is the number of standard deviations in a normal distribution PDF corresponding to the desired failure probability limit. The width of an interval function is denoted by $\alpha$. RBDO defines uncertain variables and parameters probabilistically while PBDO defines uncertainties using fuzzy sets. Several solution strategies exist for solving PBDO problems including the vertex method. The vertex method involves solving full optimizations for every combination of upper and lower boundaries corresponding to the uncertain parameters and variables. This can become very computationally expensive for problems having large numbers of uncertain variables [101]. More recently, FORM based solution strategies have been implemented in PBDO problems [90]. Unlike probabilistic uncertainty, FORM can be solved exactly for many types of convex membership functions. Methods for solving RBDO problems are reviewed in greater detail in Chapter 2.3.

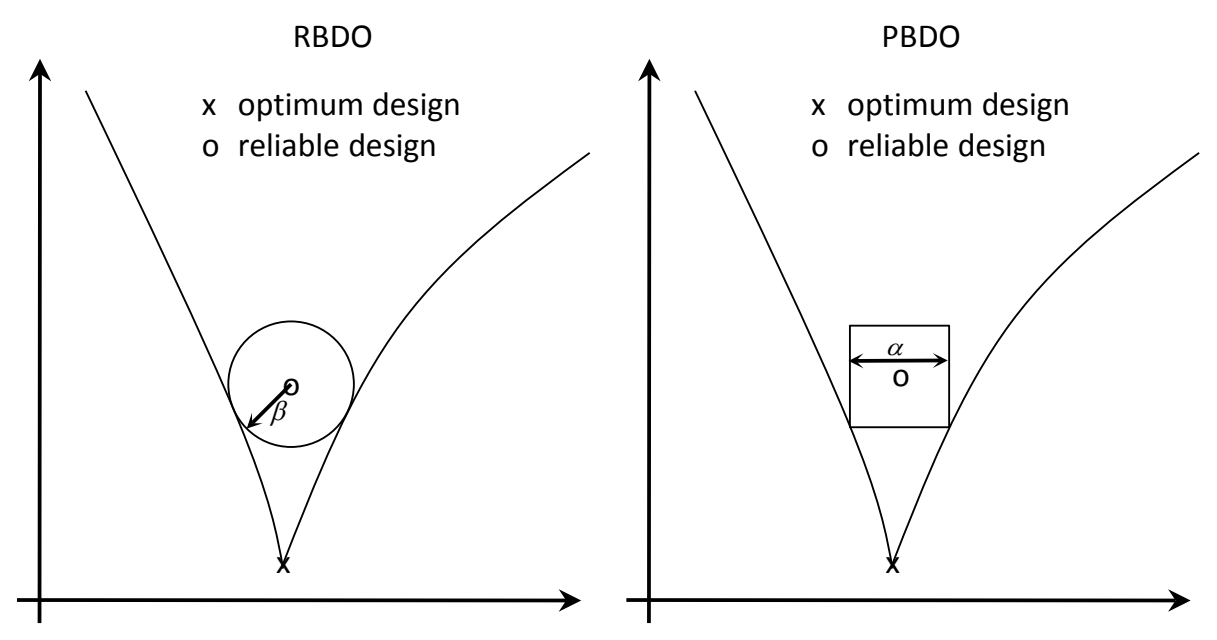

Figure 2.2.5: Reliability Based Design Optimization

It should be noted that the term reliability in the context of RBDO refers to the probability that a design lies in feasible space in optimization problems that have uncertain variables or parameters. Reliable solutions are solutions that are unlikely to violate any constraint. It does not refer to the expected quality, time-before-failure, fault tolerance, or other measures 
typically associated with the term reliability in other disciplines.

\subsection{Reliability-Based Design Optimization}

RBDO is an optimization strategy for finding reliable designs for problems that depend on uncertain design variables or parameters. Optimization solutions are considered reliable if there is a low probability that any of the specified optimization constraints are violated. The violation of any of the constraints in an optimization problem statement constitutes a failure [102]. RBDO has recently generated much interest in MDO research. It is widely viewed as a better way to deal with uncertainties in design than applying safety factors to deterministic solutions [103]. RBDO allows the influence of uncertain terms to propagate through the design optimization process, driving design changes that only affect the constraints that approach their respective boundaries.

RBDO has been applied to a wide variety of engineering problems that encounter uncertainties in material properties, manufacturing tolerances, weather conditions, and others. Thyanedar et al. proposed RBDO as a method for accounting for material defects and manufacturing tolerances in structural design [104]. Youn et al. studied vehicle crashworthiness under an uncertain impact location on a vehicle frame constructed with structural members having uncertain dimensions due to the variability in manufacturing [61]. Deb et al. solved the same crash-worthiness problem using evolutionary algorithms in order to enable handling multiple objective functions including a reliability objective.

\subsubsection{Reliability Assessment Strategies}

The most widely implemented approaches for reliability assessment are derived from FORM. All uncertain design variables and parameters are translated into normal distribution space. 
The minimum distance between the current design point and a given constraint boundary is calculated in normal space. The point along the constraint boundary at the location of closest approach is referred to as the Most Probable Point (MPP). The distance between the design point and the MPP is defined as the reliability level, $\beta$. The reliability level equates to the number of standard deviations from the mean value that the current design point lies from a constraint boundary in normal space. There are several numerical approaches for calculating the location of the MPP and the corresponding $\beta$ value. The two most common methods include the Reliability Index Approach (RIA) and the Performance Measure Approach (PMA).

\subsubsection{The Reliability Index Approach}

RIA is a direct method for calculating $\beta$ [105]. The uncertain variables and parameters are transformed into standard normal space. Uncertain parameters are probabilistic values that are not changed by the optimizer. For example, the transformation equation for a

normal distribution is the $U=x-\frac{\mu}{\sigma}$ where $U$ is the design and uncertain variable vector in normal space and $\mu$ and $\sigma$ are the mean values and standard deviations of the variables or parameters respectively. The reliability index, $\beta$, is calculated by solving the optimization problem shown in equation 2.3.1, which calculates the distance between the current design point and the closest approach of a given constraint function. The constraint number is denoted by $i$.

$$
\begin{gathered}
\text { Minimize }\|U\| \\
\text { Subject to } G_{i}(U)=0
\end{gathered}
$$


Solving 2.3.1 calculates the co-ordinates of the $M P P$ in normal space. The distance from the MPP to the design point is the reliability level, $\beta_{i}$, and is calculated by equation 2.3.2. The process is illustrated in Figure 2.3.1, where $G$ is a constraint function evaluated at normalized variable vector $U$ [106].

$$
\beta_{i} \approx\left\|U_{G_{i}=0}\right\|
$$

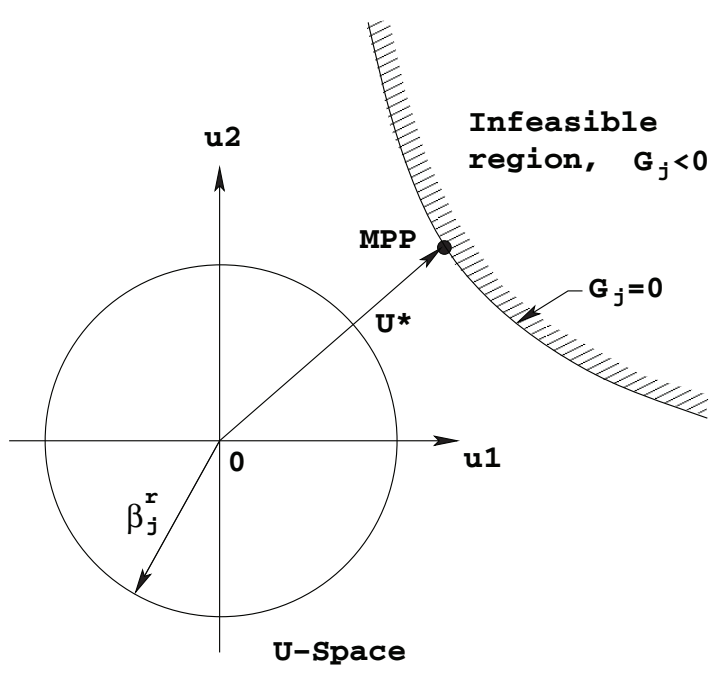

Figure 2.3.1: RIA Approach (Deb, 2007)

In an optimization scheme, $\beta_{i}$ is calculated for each constraint function. The constraints are re-formulated to constrain each $\beta_{i}$ to exceed the target reliability level. RIA has been shown to exhibit poor convergence for some problems since enforcing equality constraints on nonlinear or coarse constraint functions causes convergence problems for many optimization algorithms [107]. Despite this drawback, the RIA method has the advantage that the reliability level for each constraint can be calculated directly, unlike the PMA method, where a desired reliability level must be implicitly enforced for each constraint. A direct calculation of $\beta$ is particularly useful for when solving for a range of solutions (a Pareto front) 
showing the trade-off between the reliability level $\beta$ and some objective function. Deb et al. demonstrated this approach on an analytical problem and a vehicle crash-worthiness problem using evolutionary algorithms [106].

\subsubsection{The Performance Measure Approach}

The PMA method, introduced by Tu et al., essentially solves the inverse of the RIA optimization [107]. As shown in Figure 2.3.2, the constraint level, $G_{i}(U)$ is minimized subject to a constraint that forces the distance in normal space to the MPP to become equal to the desired reliability level $\beta$, resulting in the depth into feasible design space that must be enforced [108]. This is an improvement over the RIA method because non-linear constraint functions become a minimization problem, not an equality constraint as in the RIA method, resulting in an approach that is generally more robust and efficient for most applications $[68,109]$.

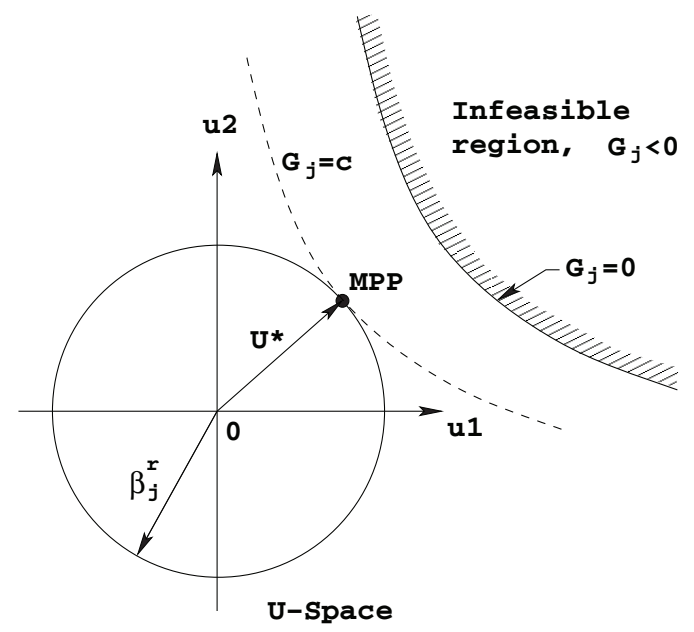

Figure 2.3.2: PMA Approach (Deb, 2007)

For aircraft conceptual design, many non-linear constraint functions are enforced. These include desired performance characteristics, stability, and others. The RIA method requires 
enforcing each constraint in turn to an equality condition. This leads to poor convergence characteristics in gradient-based optimizers, making the PMA method a more suitable approach for such problems since each constraint function is minimized in the RBDO problem objective function, not constrained to an equality condition.

$$
\begin{gathered}
\text { Minimize } G_{i}(U) \\
\text { Subject to }\|U\|=\beta_{t}
\end{gathered}
$$

\subsubsection{Reliability Based Optimization Integration Strategies}

A reliability based optimization framework integrates an optimizer that adjusts the design variables (both deterministic and uncertain) with a method for performing reliability analysis. There are three general approaches for accomplishing this. They are referred to as double-loop, sequential, and single-loop and are described as follows.

\subsubsection{Double Loop Method}

The Double Loop approach is the traditional method for integrating reliability analysis with optimization frameworks. Double loop RBDO strategies consist of an outer optimization loop that adjusts the mean values of the design variables. The objective function is evaluated at the mean values of the uncertain variables and parameters. Each optimization constraint is evaluated by carrying out either a PMA or RIA based reliability analysis every time the design variables are changed. The optimization can be constrained to reach a desired reliability level using RIA or constrained to be feasible at a specified reliability level using PMA. The block diagram for the double loop method is shown in Figure 2.3.3 [110].

Double loop methods are computationally expensive and can exhibit convergence prob- 


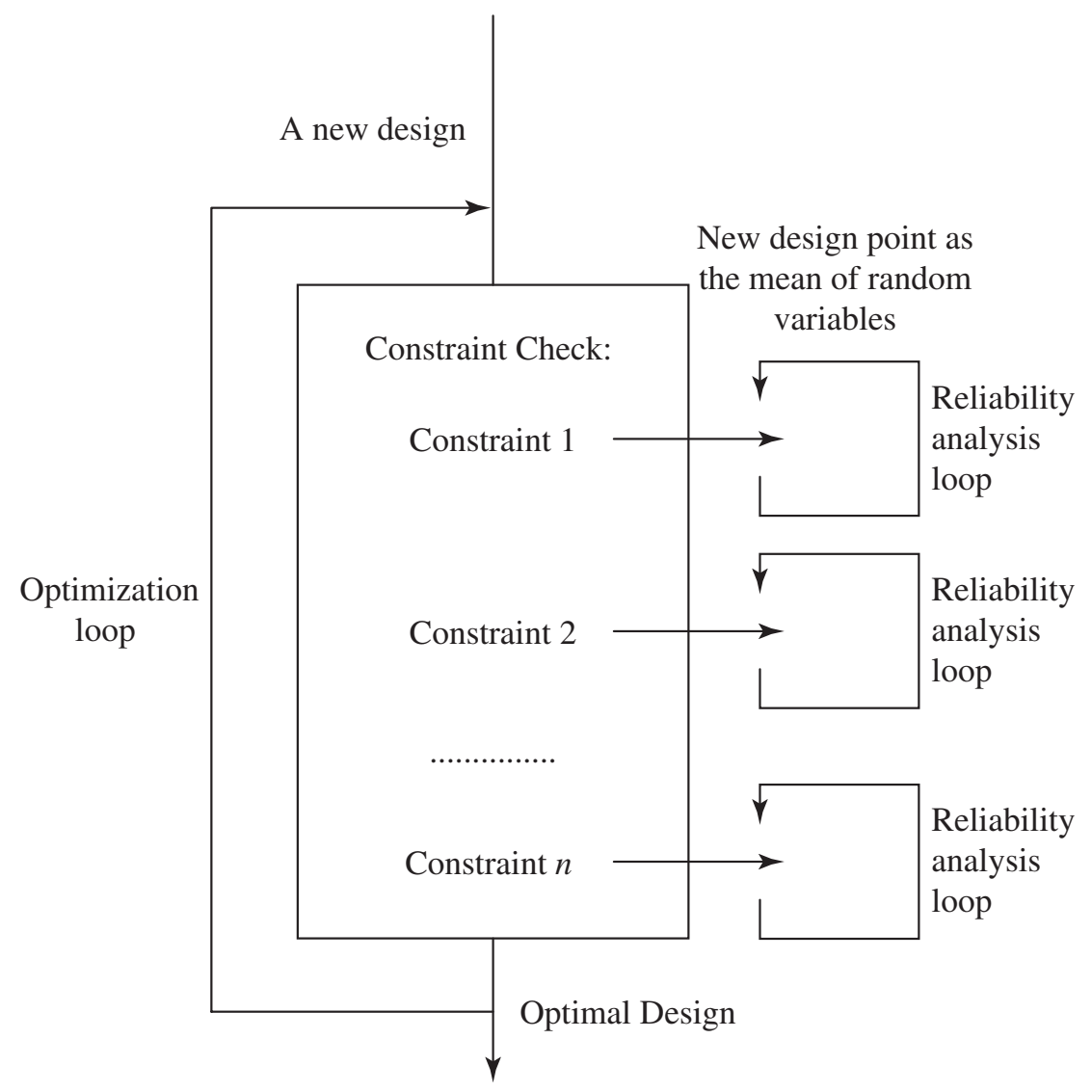

Figure 2.3.3: Double Loop Method (Shan, 2008)

lems for the following reasons. The convergence of the inner optimization loop may not be sufficiently precise for the outer loop optimizer to calculate accurate gradients [111]. Double loop methods require a full reliability analysis to be solved for every probabilistic constraint whenever the design variables are altered. This includes the small steps used for calculating gradients by finite-differencing when gradient based optimizers are used. This greatly increases the computational expense for problems that have a large number of probabilistic constraints. These drawbacks have led to the development of strategies that decouple the optimization loops into more efficient arrangements. Other alternative methods eliminate the inner reliability assessment loop by implementing gradient-based 
approximations to estimate the FORM solution at each iteration.

\subsubsection{Sequential Method}

Sequential RBDO was first proposed by Wu et al. [112, 113]. The approach was dubbed "Safety Factor Based" RBDO. Du et al. extended the concept with Sequential Optimization and Reliability Assessment (SORA), which introduced some optimizations to enhance the convergence rate [111]. Sequential RBDO schemes address the convergence problems and the computational cost associated with double loop methods by decoupling the reliability analysis optimization loops from the main problem. This is accomplished by an arrangement where a full deterministic optimization problem is solved in sequence with the reliability assessments as shown in Figure 2.3.4 [111]. The deterministic optimization provides an initial estimate of a reliable solution. This is followed by a reliability analysis optimization loop (usually PMA) for every constraint. The MPP associated with each constraint is determined. The differences between the mean values obtained by the deterministic optimization and the design variables at the MPP are calculated and stored in the shift vector, $s$. A new deterministic optimization problem is solved with modified constraint boundaries where each constraint is evaluated at a position $x+s$. Note that each constraint is evaluated at a different shift vector for a given set of design variables. The algorithm continues to alternate deterministic optimization with reliability analysis until consistency. This method has been shown to be significantly more efficient than double loop approaches $[72,111,112]$. Additionally, since the reliability analysis occurs in sequence with the system optimization rather than in a nested loop, the system optimization is less sensitive to imprecise convergence of the reliability analyses. 


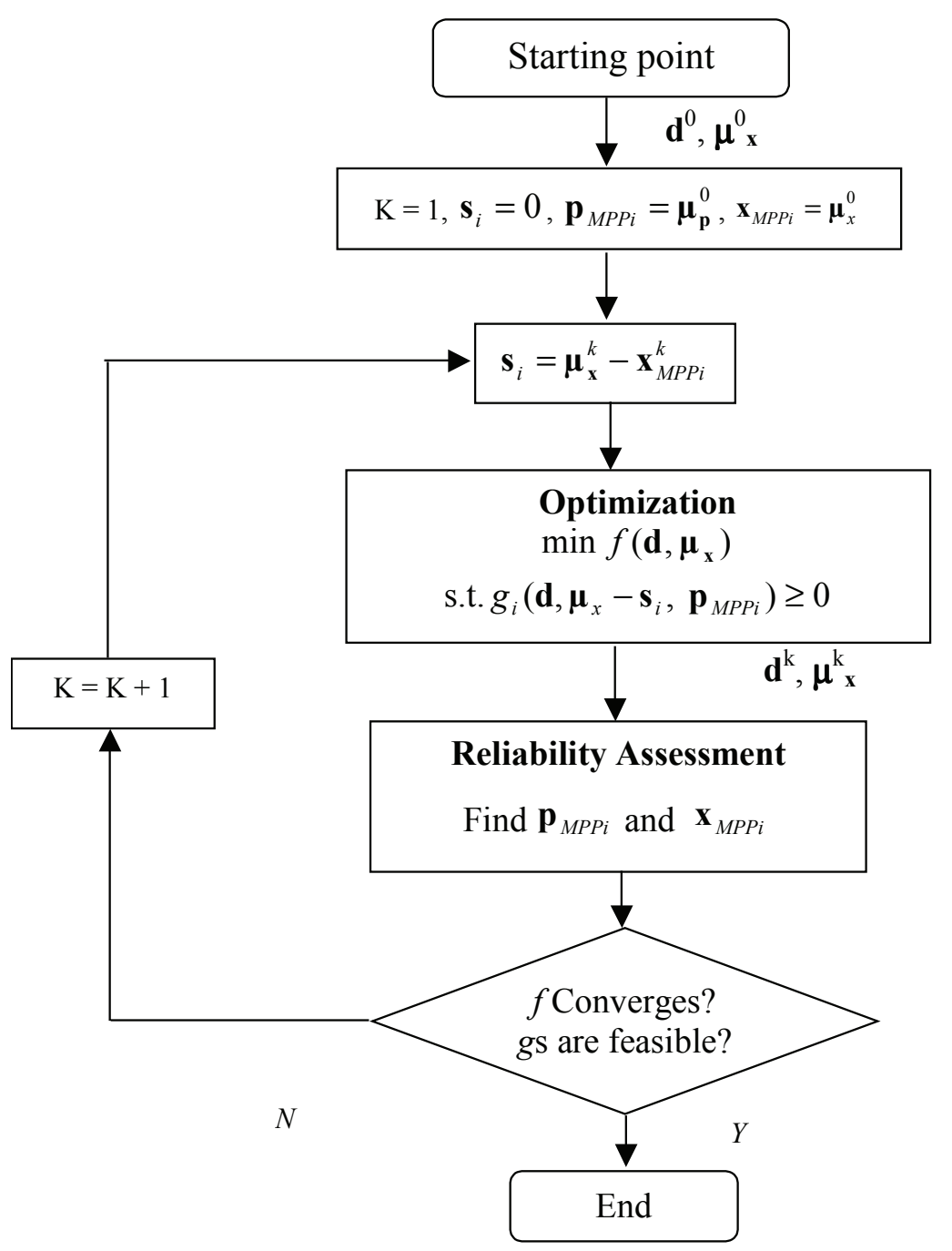

Figure 2.3.4: Sequential Method (Du, 2004)

\subsubsection{Single Loop Method}

Single loop methods eliminate the need for nested loops or sequential optimizations by implementing gradient-based approximations of the MPP for each probabilistic constraint. The approximation is updated at every iteration of the optimizer. Chen et al. introduced the Single Loop Single Variable (SLSV) method, an early example of the single loop approach [114]. Other single loop algorithms have been proposed by Kuchel et al. and others that bring the normal distribution variates as additional design variables [115]. However, 
Liang et al. reported that these methods require computationally expensive second order derivatives, and proposed an enhanced SLSV method, outlined in Figure 2.3.5, requiring no additional design variables or second order derivatives [116].

The method operates by solving a deterministic objective function subject to constraints calculated at shifted uncertain variable levels, which are updated at every iteration in gradient based optimizers. The computational effort required is similar to deterministic optimization for some problems. However, like the sequential and single loop methods, each constraint must be evaluated at different design variable states. For problems such as the optimization of a finite-element structure, the objective (minimizing mass, for example) and constraints (stresses) may be evaluated with one execution of the finite-element method. For a reliability-based optimization, the FEM model must be updated and executed independently for the objective function and all of the constraints individually, greatly increasing the number of function evaluations relative to deterministic optimization.

Several currently available design tools incorporate RBDO methods for considering uncertainty in design. DAKOTA is a design optimization tool developed by Sandia National Labs [117]. DAKOTA provides a number of optimization algorithms and tools for surrogate modeling. Recently, RBDO methods were incorporated including the RIA, PMA, and single loop approaches, simulation based methods, and second order methods $[109,117,118,119]$. DAKOTA emphasizes parallel computing and surrogate modeling to facilitate high fidelity optimization. It does not appear to contain native algorithms for solving multi-discipline RBDO problems. ProFES is a tool for integrating the uncertainty analysis capabilities of DAKOTA with FEM solvers. Wu et al. solved a single-discipline high fidelity wing box structural optimization with the ProFES package in [120]. The RBDO algorithms developed in this research emphasize multi-discipline design optimization incorporating RBDO. Both single and multi-objective optimization approaches were 
considered for solving practical aerospace design problems.

\subsection{Summary}

This chapter outlines the methodology used for modeling sources of uncertainty and describes several approaches for integrating uncertainty analysis in design optimization. Probabilistic approaches are nearly always used when sufficient statistical information is available to determine a good estimate of the PDF for each source of uncertainty. For sources of uncertainty where there is insufficient data to determine a PDF function accurately, interval and fuzzy analysis can be implemented. The boundaries and types of membership functions are developed by experts using both subjective knowledge as well as objective data. Optimization strategies that consider the influence of input uncertainty were reviewed. Robust design searches for designs that exhibit low sensitivity to variances in the uncertain variables and parameters. RBRDO finds optimum designs where the output variances of constraint functions lie completely within feasible design space. Both PBDO and RBDO are methods for finding reliable designs by evaluating the likelihood of constraint failure. In PBDO, the uncertain variables are defined using fuzzy sets or intervals. RBDO methods define uncertain variables probabilistically.

Commercial aircraft design traditionally implements statistical analysis methods in the conceptual design phase. Historical data concerning aircraft specifications is generally plentiful. Consequentially, the uncertainties that arise from using low fidelity physics and statistical methods in aircraft conceptual design can be represented probabilistically by comparing predicted performance characteristics with published data. Therefore, RBDO is a suitable approach for handling uncertain contributing analysis methods in aircraft conceptual design. 


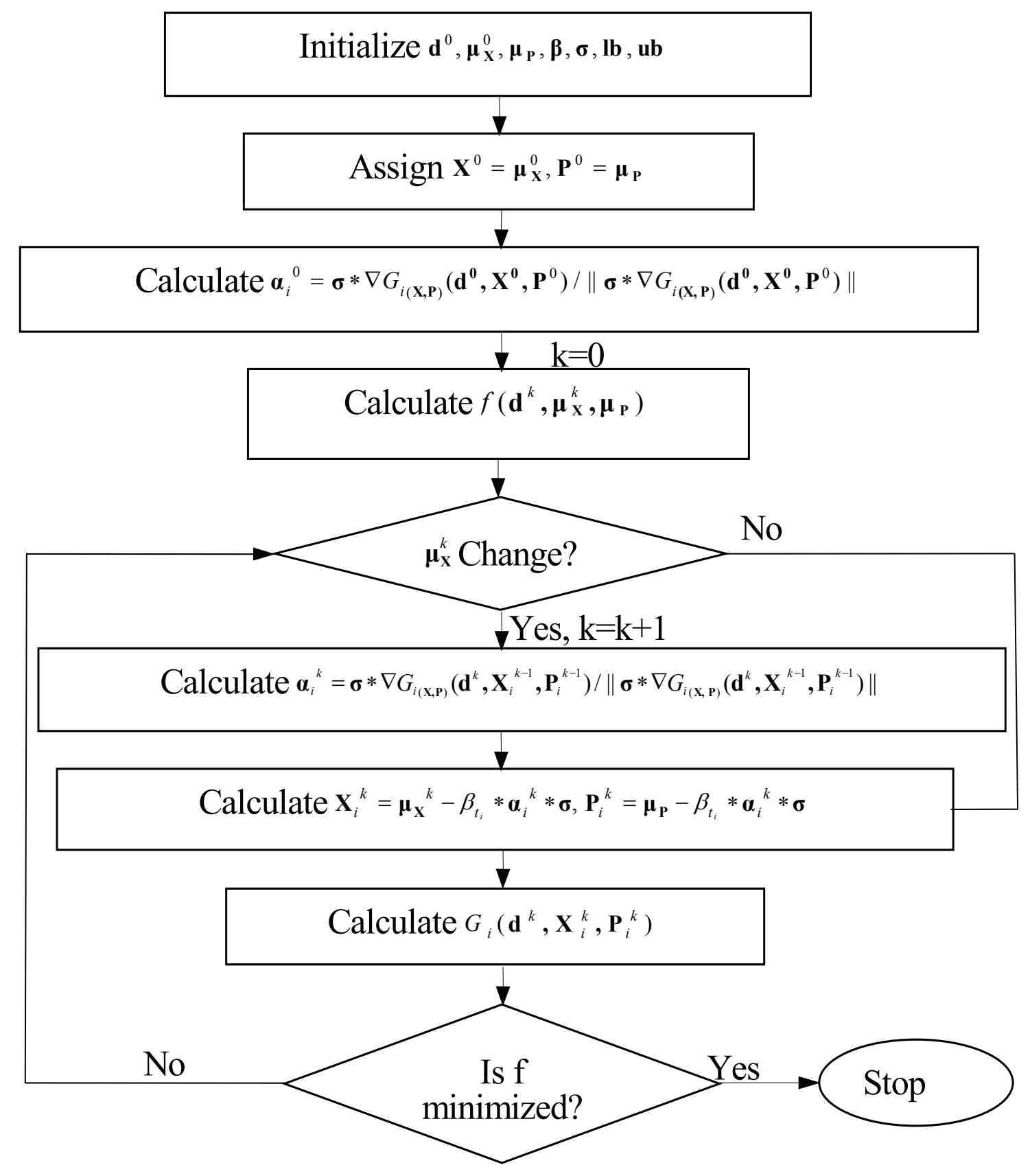

Figure 2.3.5: Single Loop Method (Liang, 2008) 


\section{Chapter 3}

\section{RyeMDO: A Multi-Discipline, Multi-Objective RBDO Package}

This chapter describes the development and validation of RyeMDO, a software package for multi-objective, multi-disciplinary reliability based design optimization. The development of RyeMDO began with the deterministic optimization of a simplified Unmanned Aerial Vehicle (UAV) conceptual design optimization case study [121, 122]. This work was later extended to multi-objective conceptual design of light jet aircraft using a GA optimizer [123]. The results were promising, but the highly simplified conceptual design equations had limitations, and the analysis of constraints such as dynamic stability, handling qualities, and control surface sizing could not be introduced without increasing the sophistication of the implemented analysis methods. Better performance simulation modules were developed and a vortex lattice method was implemented, enabling the consideration of dynamic stability constraints and control surface sizing. However, the new analysis packages introduced coupling between the disciplines, requiring the implementation of MDO methodology. A multi-discipline commercial aircraft conceptual design case study was solved 
$[124,125]$. The work was again extended to consider multiple objectives using GA with some enhancements for the purpose [40]. Again, the results were promising, but despite the enhancements, the utilized aircraft conceptual design methods still relied on some statistical equations and other simplified analysis methods. This limits the confidence designers may place in any of the results obtained from the optimizer. RyeMDO was developed to address this by introducing reliability analysis as a means of accounting for the uncertainties introduced by the approximate analysis methods commonly used in aircraft conceptual design. A method was developed whereby analysis methods are benchmarked and compared to historical data to establish their accuracy. This information is carried forward into the optimization process. The resulting designs would therefore be more conservative than those computed by deterministic optimization. However, the designer may have increased confidence that the performance predictions of the new conceptual design are more reliable.

RyeMDO is a modular package that enables the integration of several different RBDO strategies with several alternative MDO frameworks depending on what is most suitable for a given problem. Each module is generalized to work with most optimization algorithms. It is suitable for general purposes and is not limited to aircraft conceptual design problems. Figure 3.0.1 lists the main RyeMDO modules. RyeMDO was programmed in MATLAB, a programming language for mathematical computation [126].

RyeMDO contains six alternative modules for reliability assessment. The FORM based methods implemented include both PMA and RIA methods. Modified variants of the PMA and RIA methods were developed for MDO strategies such as the IDF method that require auxiliary coupling variables and constraints. The PMA and RIA optimization problems are solved via MATLAB's SQP algorithms. Two modules for MCS based reliability assessment were developed. The first evaluates the system equations and is useful for problems that consist of only fast-solving analysis methods. The second MCS based method imple- 


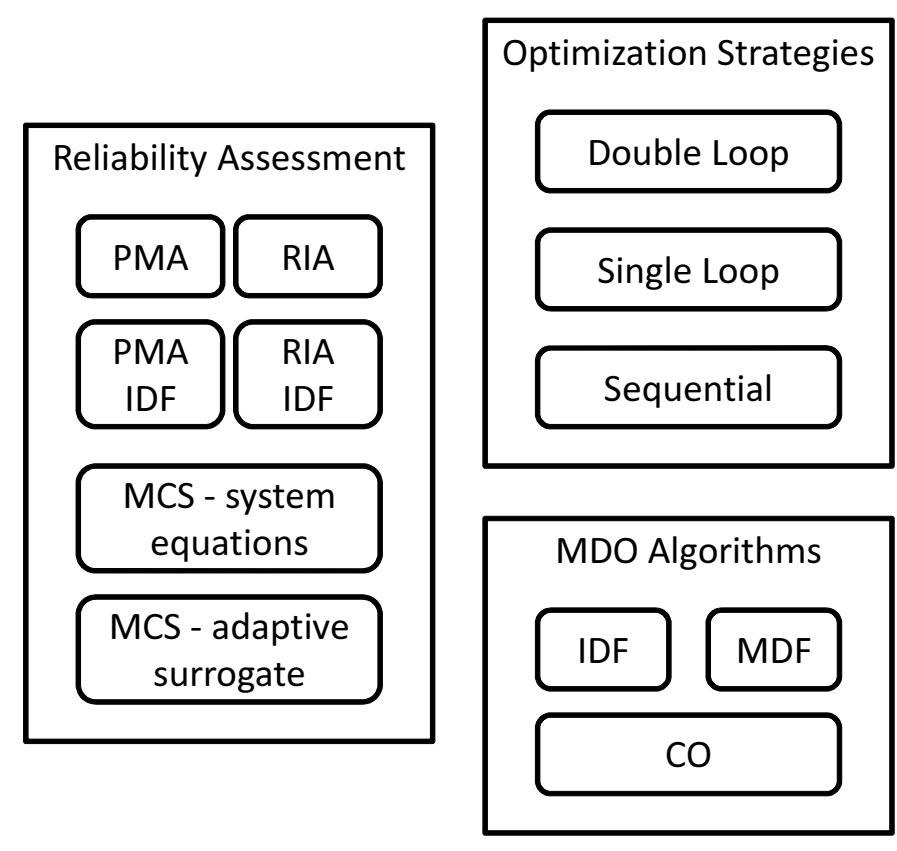

Figure 3.0.1: RyeMDO Modules

ments self-updating surrogate models that mathematically represent the constraints with respect to the uncertain variables and parameters locally around the current design point. The models are calculated by sampling a user specified number of points and are updated whenever the design variables change significantly. The method is suitable for problems where the analysis methods are too computationally expensive for MCS to be practical but too noisy or discontinuous for gradient based optimizers to be effective. Both MCS methods utilize a multi-objective GA optimizer.

Modules for the double loop, single loop, and sequential optimization strategies were developed. The double loop and sequential approaches can implement either the PMA or RIA reliability assessment methods. The single loop approach implements a gradient based approximation of the PMA method. Modules for solving multi-disciplinary problems were developed using the MDF, IDF, and CO methods. The modules can be combined with either the single loop, double loop, or sequential strategies using PMA or RIA. Many dif- 
ferent combinations of reliability assessment methods, optimization strategies, and MDO algorithms are possible. RyeMDO was designed to handle uncertainty in design variables, constant parameters, and the output of contributing analysis methods. Many well-known PDF functions are supported. Both gradient based and stochastic optimization methods can be selected.

RyeMDO is invoked by an input script that defines the parameters of the problem to be solved. First, the user must define the objective functions, constraint functions, and the discipline analysis functions. Each function must accept a vector of design variables (uncertain and deterministic), coupling variables, uncertain parameters, and any required output from the discipline analysis methods. Following the function definitions, the PDF functions for each uncertain variable and parameter must be defined. For problems with uncertain analysis methods, a database must be provided. The data must describe how the true system behaves with respect to the design variables. It may be derived from the results of physical testing, historical data, or from more exact analysis methods. RyeMDO uses the provided analysis methods to predict the database entries. The user must examine the histogram of the discrepancies between the data and predictions and select a PDF function that approximates the histogram best. Following this, the reliability assessment method must be selected. The MCS based methods implement a GA optimizer. If any of the FORM based approaches are selected, the user must select the optimization strategy - double loop, single loop, or sequential. These methods may be combined with the MDF, IDF, or CO methods for multi-discipline problems. The PMA or RIA methods implement MATLAB's SQP optimizer. The user may select either a GA optimizer or SQP for the outer optimization loops. The optimization can then be carried out with a user defined reliability level target. Alternatively, the reliability level can be introduced as a second objective function and solved by multi-objective optimization methods. This enables the user to determine 
any trends in the optimum design as the reliability level is increased. Figure 3.0.2 outlines the procedure.

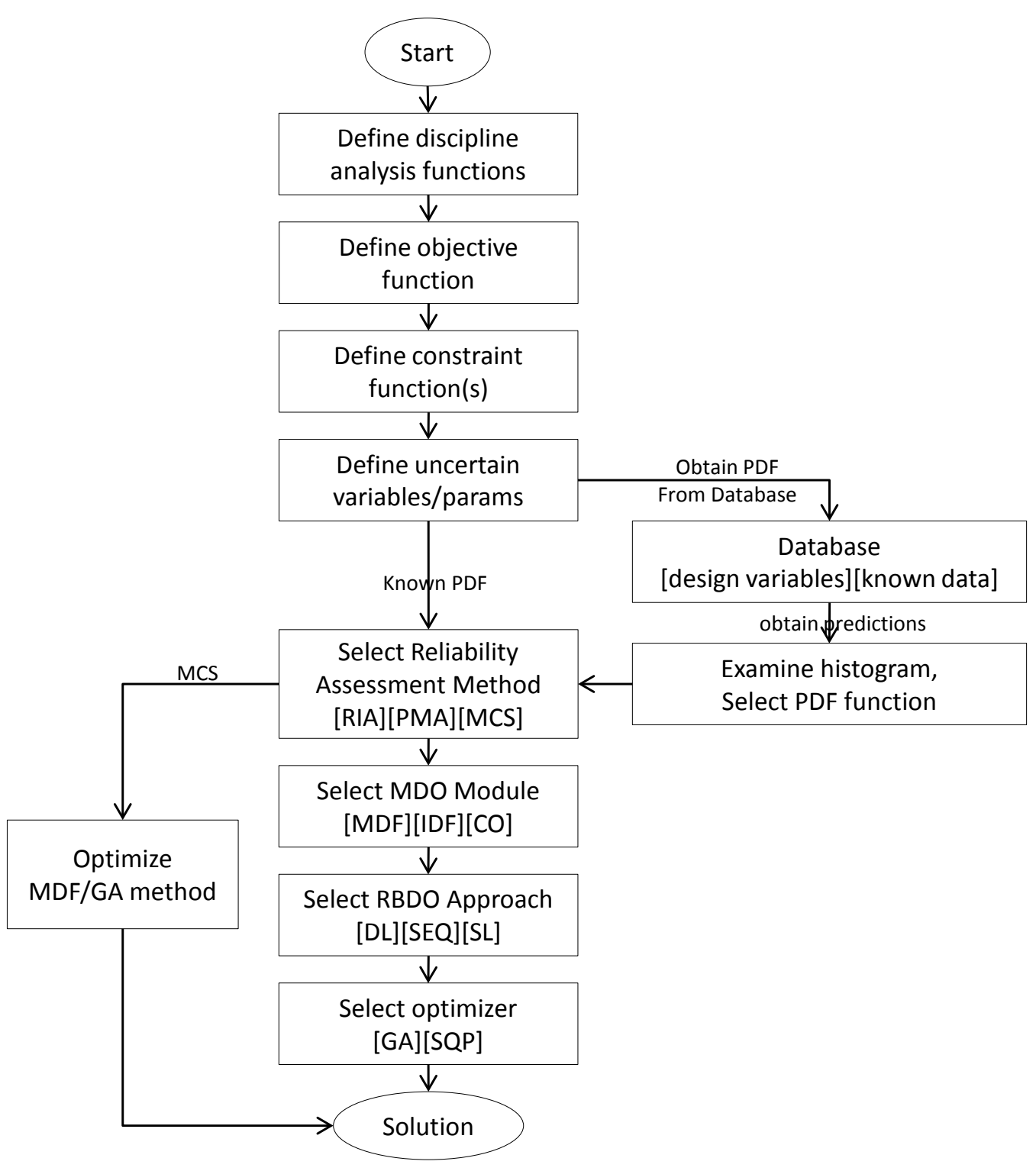

Figure 3.0.2: RyeMDO - Solution Procedure

Section 3.1 describes solution strategies for reliability based MDO problems using RyeMDO.

In Section 3.2, several well-known optimization problems are solved to validate the software and to compare the performance of many of the solution methods that can be created 
by different combinations of the RyeMDO modules.

\subsection{Reliability-Based Multi-disciplinary Design Optimiza- tion Strategies}

MDO can be simply defined as the optimization of systems that require the simultaneous solution of several contributing disciplines. RyeMDO provides modules for several MDO algorithms including MDF, IDF, and CO. Each method can incorporate several of the RBDO integration strategies discussed in Section 2.3.2. The following sections review each MDO approach and describe how the several reliability assessment strategies can be integrated.

The integration of RBDO methods with MDO problems has been the subject of some recent research $[32,61,127,128]$. However, a study of the relative performance characteristics associated with integrated RBDO-MDO methods is not currently available. Section 3.2 addresses the need to establish the most promising approaches. The accuracy, reliability, and efficiency of each approach were assessed and compared. The results of the study are carried forward to the solution of engineering case studies in Chapters 4 and 5 .

\subsubsection{MDF Method}

The MDF architecture is a single level MDO approach where every candidate design generated by the optimizer is feasible. In other words, any shared design variables and coupling variables are consistent in every discipline. This is accomplished by the introduction of a MDA loop. The MDA loop begins at a given design variable state assigned by the optimizer using an initial guess of the coupling variable vector, denoted by $y$. Each discipline is run in 
turn to obtain updated coupling variable quantities from the discipline analysis output. The loop repeats until the coupling variable vector reaches consistency. An MDA loop must be carried out whenever the design variables are altered. Most other MDO methods only guarantee feasible designs at the final optimum point and require the introduction of coupling variables to the system optimizer, significantly increasing the dimensionality of the optimization problem while possibly reducing the number of required function evaluations $[43,46,48]$. The MDA loop can implement substitution methods similar to Gauss-Siedel, the Newton-Raphson method, or other numerical approaches depending on what is most suitable for the governing equations of each discipline. The optimization problem formulation for a deterministic MDF based approach is given in equation 3.1.1 where the shared design variables and local design variables are denoted by $z$ and $x$ respectively. The coupling variables, denoted by $y$, are solved by carrying out an MDA loop every time the system and local variables are adjusted.

$$
\begin{aligned}
& \min _{z, x} f[z, y(x, y, z), x] i, j=1, \ldots, n \\
& \text { s.t. } g[z, y(x, y, z), x] \leq 0
\end{aligned}
$$

A reliability based MDF formulation can be stated as shown in equation 3.1.2 where $P$ is the probability of feasibility for each problem constraint and $p$ represents uncertain parameters (parameters that are probabilistic but have fixed mean values that remain unaltered by the system optimizer).

$$
\begin{gathered}
\left.\min _{z, x} f[\bar{z}, y(\bar{x}, y, \bar{z}), \bar{x}, \bar{p}]\right] \\
\text { s.t.P }(g[z, y(x, y, z), x, p] \leq 0) \geq P_{\text {goal }}
\end{gathered}
$$


The MDA loop serves as an interface between the optimizer and the problem disciplines. The optimizer manipulates the shared variables, $z$, and each of the discipline's local variables, $x$. The coupling variables are solved internally by the MDA loop and do not appear in the system optimizer. Any single discipline RBDO approach can therefore be applied. It should be noted that an MDA analysis may require many function evaluations and is performed whenever the design variables (deterministic or probabilistic) are updated. This applies to both the system optimization problem and each reliability assessment. As a consequence, the computational effort required for solving a multi-discipline RBDO problem is much greater than that of single discipline optimization. RyeMDO's MDF block can be integrated with any of the single discipline RBDO solver blocks directly, since all coupling variables are handled internally, and are not visible to the system optimizer.

\subsubsection{IDF Method}

The IDF method was developed to eliminate the need for the computationally costly MDA loop by removing the requirement of feasibility for every design evaluated by the optimizer. Design feasibility, or rather the consistency of the coupling and shared variables between disciplines, is only guaranteed at the final optimum point. This is accomplished by introducing the coupling variables as additional design variables in the system optimizer to serve as preliminary estimates. New auxiliary constraints are introduced that force the discrepancy between the discipline responses and the estimated values to vanish at the optimum point. The algorithm block diagram is shown in 2.1.3. The deterministic problem formulation for the IDF method is given in equation 3.1.3. The coupling variables, $y$, are evaluated by solving each discipline using the estimates of the coupling variable states, $y^{\prime}$, 
established by the system optimizer.

$$
\begin{gathered}
\min _{z, x} f\left[z, y\left(x, y^{\prime}, z\right), x\right] i, j=1, \ldots, n \\
\text { s.t. } g\left[z, y\left(x, y^{\prime}, z\right), x\right] \leq 0 \\
y^{\prime}-y\left(x, y^{\prime}, z\right)=0
\end{gathered}
$$

Performance comparisons of MDO methods consistently report IDF as being both highly efficient and stable for small and medium scale problems [43, 46, 48]. However, convergence issues have been reported for problems having a very large number of coupling variables and/or local design variables due to the increase in dimensionality of the system optimizer and the addition of the equality compatibility constraints [43]. The coupling variables are also introduced to the system optimization variables for IDF-based RBDO algorithms with corresponding auxiliary constraints. However, the same must be done for every reliability analysis, as shown in equation 3.1.4 for PMA based reliability analysis.

$$
\begin{gathered}
\min G_{i}(U) \\
\text { s.t. }\|U\|=\beta_{t} \\
\text { becomes } \\
\min G_{i}\left(U, y^{\prime}\right) \\
\text { s.t. }\|U\|=\beta_{t} \\
y^{\prime}-y\left(x, y^{\prime}, z\right)
\end{gathered}
$$

Incorporating the revised reliability analysis loop, IDF formulations are possible using both double loop and sequential RBDO strategies. RyeMDO contains IDF modules for both the double-loop and sequential RBDO strategies. The user may select an IDF-based outer 
optimization loop and an IDF-based implementation of the RIA or PMA reliability analysis methods. Alternatively, the user may opt to use an IDF based outer loop with an MDF based reliability assessment loop. This hybrid arrangement can be advantageous when the number of coupling variables is large compared to the number of uncertain variables and parameters.

\subsubsection{CO Method}

The CO method is a multi-level MDO strategy. Each discipline is managed by an independent local optimizer. For a given discipline, the local optimizer minimizes the discrepancies between any local coupling and shared variables with those established by the system optimizer. The discipline constraints are also enforced. The system-level optimizer minimizes the main objective as a function of only shared design variables and coupling variable estimates as shown in Figure 2.1.5. Local variables only appear in the discipline-level optimizations. For this reason, the dimensionality of the system optimization problem is substantially reduced for problems that have large numbers of local design variables. Each discipline optimizer or RBDO integration strategy can be specifically chosen according to what works best on the governing equations of each discipline. If the system optimizer is gradient-based, the local optimization problems must be sensitive to small changes to the design variables. This requires consistent and precise convergence of each local optimization. Otherwise, the determination of gradients by finite-differencing becomes impossible. Since most RBDO methods require additional inner-loops or sequential optimizations, the degree of precision required by a gradient based system optimizer can be difficult to attain. Performance comparisons consistently show $\mathrm{CO}$ to be at a disadvantage with problems that do not have large numbers of local variables $[43,46,48]$ since variables shared by two or more disciplines must be solved at every discipline level, greatly increasing the 
dimensionality of the sub-problems [16]. However, $\mathrm{CO}$ has been shown to be competitive or advantageous for problems that have large numbers of local variables [129]. The deterministic problem formulation for the system optimization and local optimizations are shown in equations 3.1.7 and 3.1.8 where the index $i$ denotes the discipline number, $A$ is the auxiliary compatibility constraint function, and the subscript $S L$ denotes the system level variable values.

$$
\begin{gathered}
\min _{z_{S L}, y_{S L}} f\left(z_{S L}, y_{S L}\right) \\
\text { s.t. } A_{i}\left(z_{S L}, z_{i}^{*}, y_{S L}, y_{i}^{*}\right)=0 \\
\text { where } \\
A_{i}=\sum\left(z_{S L}-z_{i}\right)^{2}+\sum\left(y_{S L}-y_{i}\right)^{2} \\
\min _{z, y, x} A_{i}\left[z_{S L}, z_{i}, y_{S L}, y_{i}\left(x_{i}, y_{S L}, z_{i}\right)\right] \\
\text { s.t. } g_{i}\left[z_{i}, x_{i}, y_{i}\left(x_{i}, y_{S L}, z_{i}\right)\right] \leq 0
\end{gathered}
$$

The integration of RBDO strategies with CO is relatively straightforward. Since the problem objective functions are always evaluated at the mean value of uncertain variables and parameters, the system level objective function remains the same for reliability-based optimization as for deterministic optimization. Since compatibility between disciplines is enforced by the objective function of each local optimization, auxiliary constraints do not appear in the local optimization problem statements. Therefore, there is no need to modify the reliability analysis with coupling variables and compatibility constraints. The system optimization is given in equation 3.1.7. The RBDO formulation of a given local optimiza- 
tion is given in equation 3.1.8.

$$
\begin{gathered}
\min _{z_{S L}, y_{S L}} f\left(z_{S L}, y_{S L}, \bar{p}\right) \\
\text { s.t. } A_{i}\left(z_{S L}, z_{i}^{*}, y_{S L}, y_{i}^{*}, \bar{p}\right)=0 \\
\text { where } \\
A_{i}=\sum\left(z_{S L}-z_{i}\right)^{2}+\sum\left(y_{S L}-y_{i}\right)^{2} \\
\min _{z, y, x} A_{i}\left[z_{S L}, \bar{z}_{i}, y_{i}\left(\bar{x}_{i}, \bar{y}_{S L}, \bar{z}_{i}\right), \bar{p}\right] \\
\text { s.t.P }\left(g_{i}\left[z_{i}, x_{i}, y_{i}\left(x_{i}, y_{S L}, z_{i}\right), p\right] \leq 0\right) \geq P_{\text {goal }}
\end{gathered}
$$

The system level problem is the same as a deterministic $\mathrm{CO}$ formulation where the uncertain variables and parameters are evaluated at their mean values. Any of the singlediscipline RBDO implemented in RyeMDO can be used to solve the discipline level optimizations with no modification. Different RBDO strategies can be used for each discipline if necessary. RyeMDO's CO block enables the user to select distinct RBDO strategies for every discipline considered. The system level optimization may be solved using SQP or genetic algorithms.

\subsection{Validation and Benchmarking}

This section describes the validation of the RyeMDO modules on several test problems. RyeMDO supports several reliability assessment methods, optimization strategies, and MDO algorithms. The modules can be combined in many ways, resulting in a wide variety of possible RBDO-MDO strategies. In addition to validating each approach, it is 
important to identify which RBDO-MDO strategies are the most promising approaches for solving engineering problems such as aircraft conceptual design optimization. Well-known analytical RBDO test problems were solved to validate the performance and accuracy of each RyeMDO module. The analytical test problems are smooth and can be quickly and accurately solved. Truss optimization problems were developed and solved to evaluate each approach when the objective and constraint functions are not as smooth or accurate as the analytical problems. The following sections describe the validation and benchmarking of the single-discipline RBDO approaches and the integration of RBDO approaches with several MDO methods.

\subsubsection{Single Discipline Analytical Optimization}

The following mathematical example given in equation 3.2.1 is a common test problem in RBDO literature [130].

$$
\begin{gathered}
\min f=\bar{x}_{1}+\bar{x}_{2} \\
\text { st. } P\left[G_{j}(x) \geq 0\right] \geq P_{\text {goal }, j}, j=1,2,3 \\
G_{1}(x)=x_{1}^{2} x_{2} / 20-1 \\
G_{2}(x)=\left(x_{1}+x_{2}-5\right)^{2} / 30+\left(x_{1}-x_{2}-12\right)^{2} / 120-1 \\
G_{3}(x)=80 /\left(x_{1}^{2}+8 x_{2}+5\right)-1 \\
x_{1} \sim N\left(x_{1}, 0.3\right), x_{2} \sim N\left(x_{2}, 0.3\right)
\end{gathered}
$$

It is a non-linear, single discipline RBDO problem with two uncertain variables. The problem was solved with the double loop method using both RIA and PMA, the sequential method with PMA, and the single-loop method. The problem was solved at $\beta$ values of 1 to 5. These results are shown in Figure 3.2.1, and clearly shows how the solutions 
are pushed from the constraint boundaries $G_{1}, G_{2}$, and $G_{3}$ as the reliability level $\beta$ is increased, yielding more conservative solutions. The algorithm performance was compared at $\beta=3$ for all three approaches. The results are shown in Table 3.1. The results compare the relative performance and accuracy of each method. The double loop and sequential methods all yield nearly identical solutions. However, the PMA based approaches clearly solve the optimization problem more efficiently. The Sequential/PMA method required significantly fewer function evaluations than the double loop approaches. The single loop approach required significantly fewer function evaluations to solve the mathematical problem. However, the results tend toward less agreement with the PMA and RIA solutions as the reliability level increases. Both the RIA and PMA methods converge to the exact FORM solution without additional approximations while the single loop method solves an approximate solution to FORM, causing a loss in accuracy.

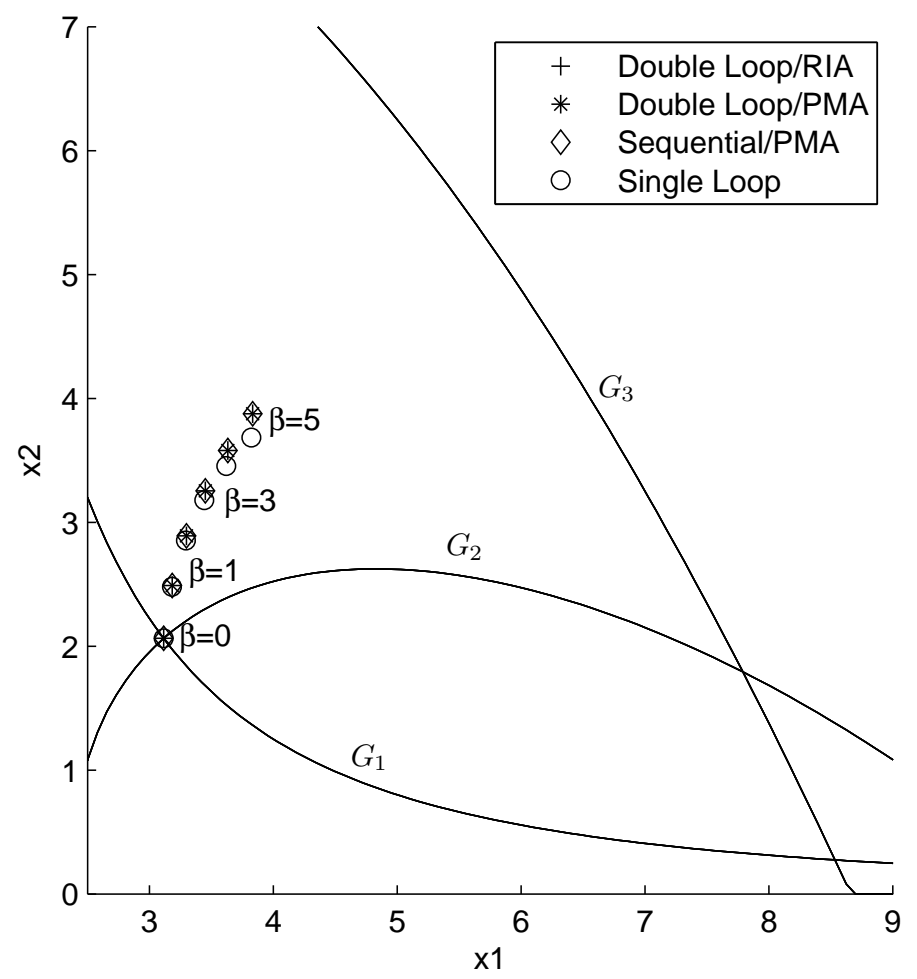

Figure 3.2.1: Single Discipline Example 
Table 3.1: Algorithm Performance Comparison

\begin{tabular}{lllll}
\hline $3 \sigma$ Solution & $f_{\min }$ & $x_{1}$ & $x_{2}$ & $n_{\text {evals }}$ \\
\hline Deterministic & 5.1769 & 3.1134 & 2.0636 & 16 \\
RIA/Double Loop & 6.7257 & 3.4391 & 3.2866 & 1530 \\
PMA/Double Loop & 6.7043 & 3.4506 & 3.2537 & 1004 \\
PMA/Sequential & 6.7043 & 3.4506 & 3.2537 & 651 \\
Single Loop & 6.6198 & 3.4413 & 3.1785 & 16 \\
\hline
\end{tabular}

The stability, speed, and accuracy of each algorithm were evaluated by running the optimization problem over a grid of 100 starting points between $[0,0]$ and $[10,10]$. The results of this evaluation are shown in Table 3.6. A failed run was defined as an optimization that failed to converge to within $10 \%$ of the true exact FORM solution of 6.7043. Clearly, the RIA/Double Loop method was the least reliable of the methods tested, with 71 out of 100 runs failed and an average error of $6.11 \%$ for the converged solutions. The method was especially problematic when dealing with infeasible starting points. The PMA/Double Loop method was found to be reliable, with no failed runs. It required substantially fewer function evaluations and lower solution times than the RIA/Double Loop method. The PMA/Sequential method was also reliable, with only 5 of 100 runs failing to converge. The average and median relative error was found to be the lowest of the methods tested. The Single Loop method exhibited by far the fastest solution times and did not fail to converge regardless of the starting point. However, the solutions were found to lie some distance from the exact FORM solution. Figure 3.2.1 indicates that this discrepancy worsens with increasing reliability levels for the analytical problem.

Analytical problems are useful for establishing some general performance comparisons. However, they are usually smooth functions that can be solved directly with great accuracy. For practical problems, the level of precision is necessarily reduced since the objective and constraint functions are likely to consist of analysis methods that use discretization or nu- 
Table 3.2: Algorithm Stability Comparison

\begin{tabular}{llllll}
\hline method & $\begin{array}{l}\text { number of } \\
\text { failed runs }\end{array}$ & $\begin{array}{l}\text { average } \\
\% \text { error }\end{array}$ & $\begin{array}{l}\text { median } \\
\% \text { error }\end{array}$ & $\begin{array}{l}\text { average } \\
\text { time (s) }\end{array}$ & $\begin{array}{l}\text { average } \\
\text { evals }\end{array}$ \\
\hline RIA/Double Loop & 71 & 6.11 & 0.319 & 4.1316 & 13276 \\
PMA/Double Loop & 0 & 3.77 & $2.97 \mathrm{E}-5$ & 2.7277 & 3862 \\
PMA/Sequential & 5 & $2.54 \mathrm{E}-5$ & $2.54 \mathrm{E}-5$ & 0.5805 & 1648 \\
Single Loop & 0 & 1.93 & 1.25 & 0.3343 & 145 \\
\hline
\end{tabular}

merical solutions. Increasing the level of precision for such methods increases the computational effort required to obtain a solution. An 18 bar truss optimization problem is given in equation 3.2.2. The FEM based stress constraints are less smooth than the analytical example, better approximating the characteristics of practical engineering problems.

\subsubsection{Single Discipline Truss Optimization}

An 18 bar truss example, adapted from a deterministic problem proposed in [131], was solved to assess the stability and performance of the RBDO approaches on a problem with less desirable properties than the preceding analytical example. The problem objective function was set to minimize the mass of the truss structure while keeping the maximum tensile or compressive stresses in every member below the ultimate stress limit or buckling stress limit. For different designs, the critical truss element will move about the structure, resulting in a somewhat rough constraint function compared to the analytical example. The initial truss structure is shown in Figure 3.2.2, where the $\mathrm{x}$ and $\mathrm{y}$ axis units are in inches. The truss was optimized considering uncertain loading and uncertain material strength.

The problem formulation is given by equation 3.2.2. The design variables were defined as 


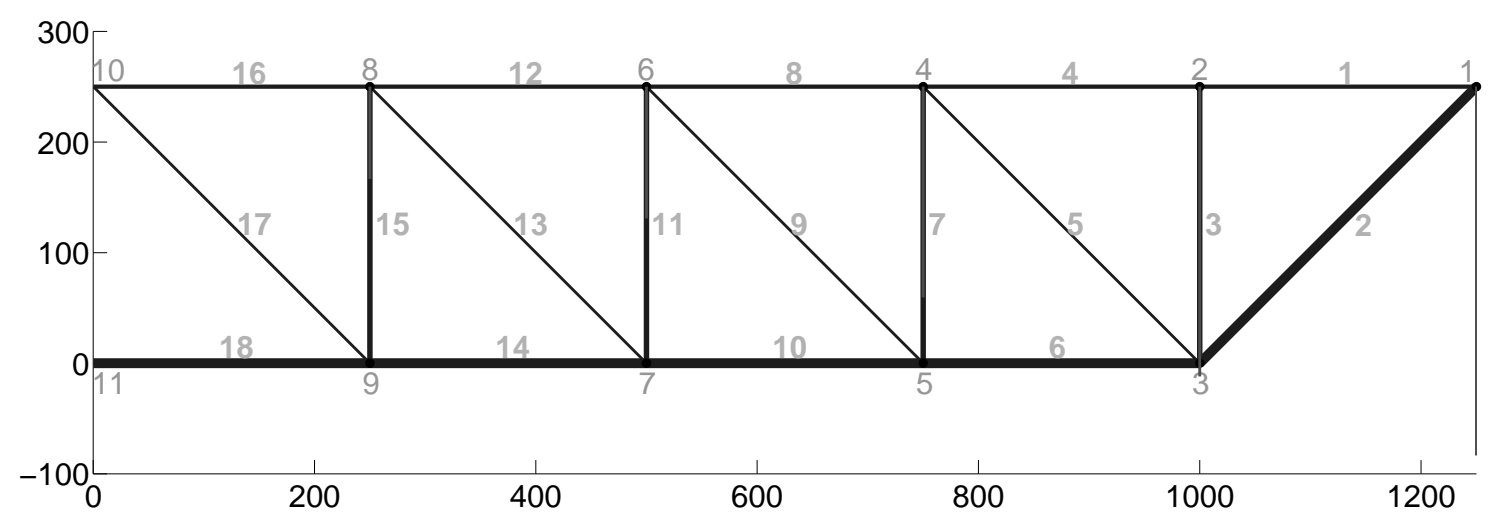

Figure 3.2.2: 18 Bar Truss

4 element area variables and 8 variables defining the co-ordinates of the lower nodes.

$$
\begin{gathered}
\min \text { mass }=f(x) \\
\text { st. } P\left[\sigma_{t} \leq \sigma_{\text {max }}\right] \geq P_{\text {goal }} \\
P\left[\sigma_{c} \leq \sigma_{b}\right] \geq P_{\text {goal }} \\
F \sim N(5000,500) \mathrm{lb} \\
\sigma_{\max } \sim N(20000,2000) \mathrm{psi} \\
\sigma_{b}=\frac{-K_{i} E_{i} A_{i}}{L_{i}^{2}}
\end{gathered}
$$

The variable definitions are shown in Table 3.3 where $x$ denotes the design variable vector, $A$ represents the element areas, and $X$ and $Y$ are the co-ordinates of the lower truss nodes. Two constraints were considered: tensile stress and buckling stress. The ultimate stress, $\sigma_{\text {max }}$, was assumed to be normally distributed $(N)$. A random, normally distributed uniform force was applied to nodes $1,2,4,6$, and 8 . The buckling stress, $\sigma_{b}$, was defined by the Euler buckling equation, given by equation 3.2.3. The elastic modulus, $E$, was assumed to be $1.0 E+7 \mathrm{psi}$. The buckling coefficient, $K$ was assumed to be 4.0 . The element length is 
denoted by $L$. The material density was assumed to be $0.1 \mathrm{lb} / \mathrm{i} n^{3}$. The required probability of feasibility, $P_{\text {goal }}$ was defined as the probability corresponding to a reliability index of 6 , or a failure probability of approximately $6.1 \times 10^{-9}$.

Table 3.3: 18 Bar Truss Design Variables

\begin{tabular}{cl}
\hline variable & definition \\
\hline$x_{1}$ & $A_{1}, A_{4}, A_{8}, A_{12}, A_{16}$ \\
$x_{2}$ & $A_{2}, A_{6}, A_{10}, A_{14}, A_{18}$ \\
$x_{3}$ & $A_{3}, A_{7}, A_{11}, A_{15}$ \\
$x_{4}$ & $A_{5}, A_{9}, A_{13}, A_{17}$ \\
$x_{5}$ & $X_{3}$ \\
$x_{6}$ & $Y_{3}$ \\
$x_{7}$ & $X_{5}$ \\
$x_{8}$ & $Y_{5}$ \\
$x_{9}$ & $X_{7}$ \\
$x_{10}$ & $Y_{7}$ \\
$x_{11}$ & $X_{9}$ \\
$x_{12}$ & $Y_{9}$ \\
\hline
\end{tabular}

Figure 3.2.3 shows the deterministic result and the reliable result obtained with the sequential approach. The other methods produced designs with very similar geometry and therefore are not shown. The performance of each RBDO approach is shown in Table 3.4.

Table 3.4: Algorithm Performance Comparison

\begin{tabular}{lcc}
\hline $6 \sigma$ Solution & mass $(\mathrm{kg})$ & $n_{\text {evals }}$ \\
\hline Deterministic & 1074 & 6920 \\
RIA/Double Loop & \multicolumn{2}{c}{ fails to converge } \\
PMA/Double Loop & 2892 & 54171 \\
PMA/Sequential & 2779 & 31948 \\
Single Loop & 3166 & 11360 \\
\hline
\end{tabular}

Referring to Table 3.4, the single loop method once again provided the most computationally efficient approach. Of the exact FORM based methods, the sequential approach was again the most efficient. Obtaining converged solutions for both the RIA and PMA based 


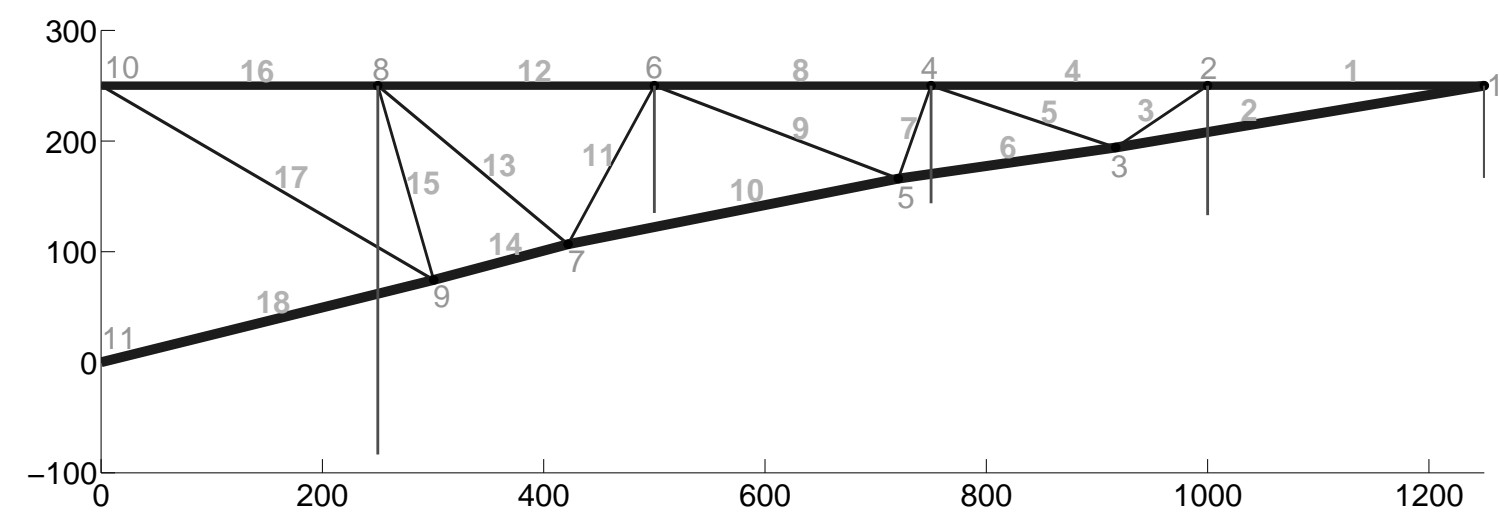

(a) Deterministic Solution

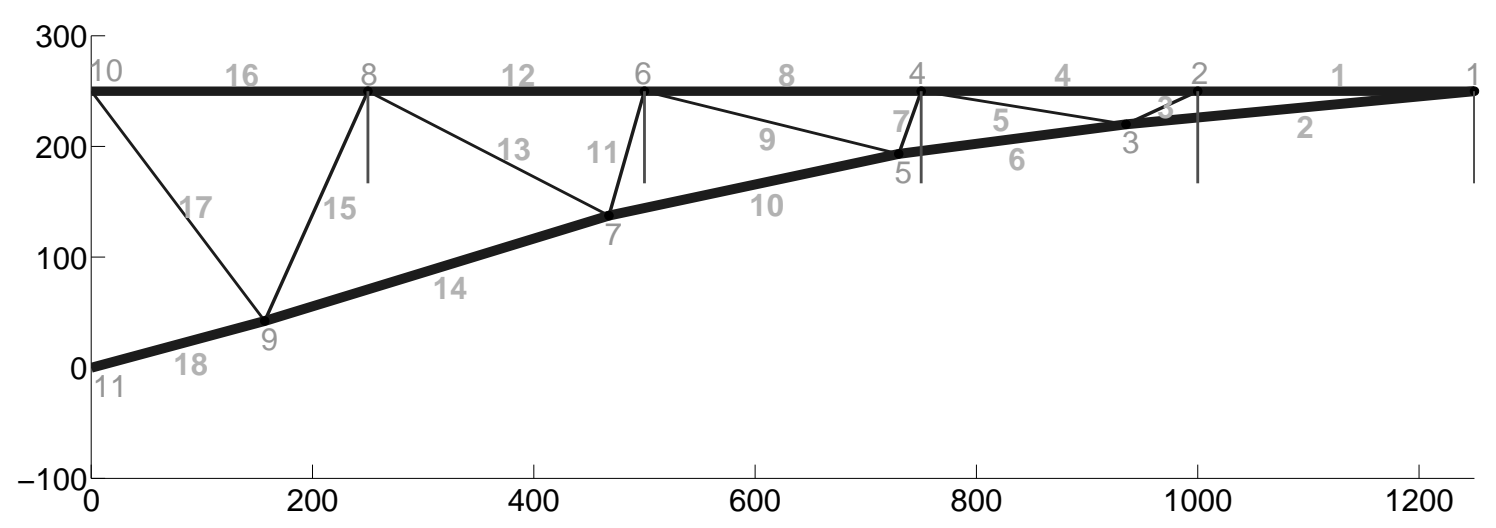

(b) Reliable Solution at $6 \sigma$

Figure 3.2.3: 18 Bar Truss Solution

double loop approaches was problematic. A solution was not obtained with the RIA/double loop approach. This appeared largely due to inconsistent convergence of the inner reliability assessment loop. Recall that double loop approaches are sensitive to the precision of the inner reliability assessment loops, which must be solved for every constraint evaluation. Optimization algorithms that utilize finite-differencing to obtain the constraint gradients will not function correctly when the inner reliability analysis loop cannot be solved with sufficient precision and sensitivity to small design variable steps. Although a solution for the PMA/double loop method was eventually obtained, the convergence of this approach was somewhat inconsistent. The convergence tolerance of the outer optimization loop had to be relaxed, and the starting vector carefully chosen for the algorithm to converge. The 
sequential approach was reliable, converging successfully with any attempted starting vector. Since the reliability assessment is solved sequentially with the optimization of the main problem, the precision of the reliability assessment does not affect the calculation of gradients by the system optimizer. This characteristic explains the good convergence characteristics of the sequential approach. The single loop approach consistently arrived at a solution. However, like the previous example, the solution of the single loop approach was found to deviate from the other solutions, consistently returning a larger optimum mass than the PMA based methods.

\subsubsection{Multi-Discipline Analytical Optimization}

A multi-discipline analytical problem from Ahn et al., given in equation 3.2.4, was used to validate the accuracy and relative efficiency of each RBDO-MDO approach implemented in RyeMDO. The uncertain variables $x_{1}$ and $x_{2}$ were assumed to have a coefficient of variation $(\mathrm{COV})$ of 0.04 with mean values assigned by the optimizer. The $\mathrm{COV}$ is defined as the ratio of the standard deviation, $\sigma$, to the mean value $\mu$, of a random variable.

$$
\begin{gathered}
\min f=-\left(\bar{x}_{1}-6\right)^{3}+y_{1}^{2}-\exp \left(-\frac{y_{1}}{y_{2}}\right) \\
y_{1}=x_{1}^{2}+y_{2} / 2 \\
g_{1}=-y_{2}+\exp \left(y_{1} / y_{2}+2.2 x_{1}\right) \\
y_{2}=x_{1}+x_{2}+\left(3 x_{1} x_{2}\right) / y_{1} \\
g_{2}=y_{2}-y_{1}-\left(x_{1}+1\right)^{2}-\left(x_{2}-4\right)^{3}
\end{gathered}
$$

The problem was solved for 8 different RBDO-MDO strategies at a reliability level of $3 \sigma$. The optimum points calculated by each approach as well as the number of function evaluations for each discipline are shown in Table 3.5. The starting vector was $x_{0}=[4,4]$ 
and the algorithm convergence tolerance was $10^{-5}$.

Table 3.5: Algorithm Performance Comparison

\begin{tabular}{lccccc}
\hline $3 \sigma$ Solution & $f_{\min }$ & $x_{1}$ & $x_{2}$ & \multicolumn{2}{c}{$n_{\text {evals }}$} \\
& & & & Dis 1 & Dis 2 \\
\hline MDF/Deterministic & 115.7971 & 1.6478 & 3.004 & 68 & 68 \\
MDF/DBL/PMA & 119.6556 & 1.5975 & 2.8056 & 16474 & 16474 \\
MDF/SLP & 119.9417 & 1.6559 & 2.9129 & 1495 & 1495 \\
MDF/SEQ/PMA & 119.6832 & 1.6208 & 2.8434 & 7413 & 7413 \\
IDF/SEQ/PMA & 119.4876 & 1.6290 & 2.8328 & 1228 & 1228 \\
IDF/DBL/PMA & 119.4464 & 1.6005 & 2.7869 & 23765 & 23765 \\
CO/SEQ/PMA & 119.1466 & 1.5930 & 2.7471 & 38958 & 34781 \\
CO/DBL/PMA & 119.1579 & 1.6000 & 2.7584 & 333623 & 201204 \\
CO/SLP & 119.0822 & 1.6300 & 2.7951 & 56338 & 186732 \\
\hline
\end{tabular}

Table 3.5 indicates that the most efficient method for solving the analytical problem was achieved by the IDF method with a sequential RBDO strategy. Also very efficient was the MDF method with a single-loop RBDO strategy. The CO based strategies did not perform well. The starting vector for the $\mathrm{CO}$ based double loop approach had to be very carefully chosen to obtain a converged solution, and nearly always failed to converge. However, it should be noted that the analytical optimization is a small scale problem and is not one where the advantages of the CO method described in Section 3.2 would be apparent.

The stability of each RBDO approach was assessed by solving the optimization from a grid of 100 evenly spaced points. The CO/DBL/PMA approach was omitted since only one converged solution was able to be obtained. The starting points ranged from $[0,0]$ to $[5,5]$. Since the RBDO/MDO solution strategies involve solving multiple nested loops within the optimization, more scatter in the calculated optimum point was observed than the previous deterministic optimization examples. The box-plot in Figure 3.2.4 shows the scatter in the predicted optimum point of each algorithm based on optimization runs with a target reliability index of $3 \sigma$ and a function and constraint tolerance of $10^{-5}$. The me- 


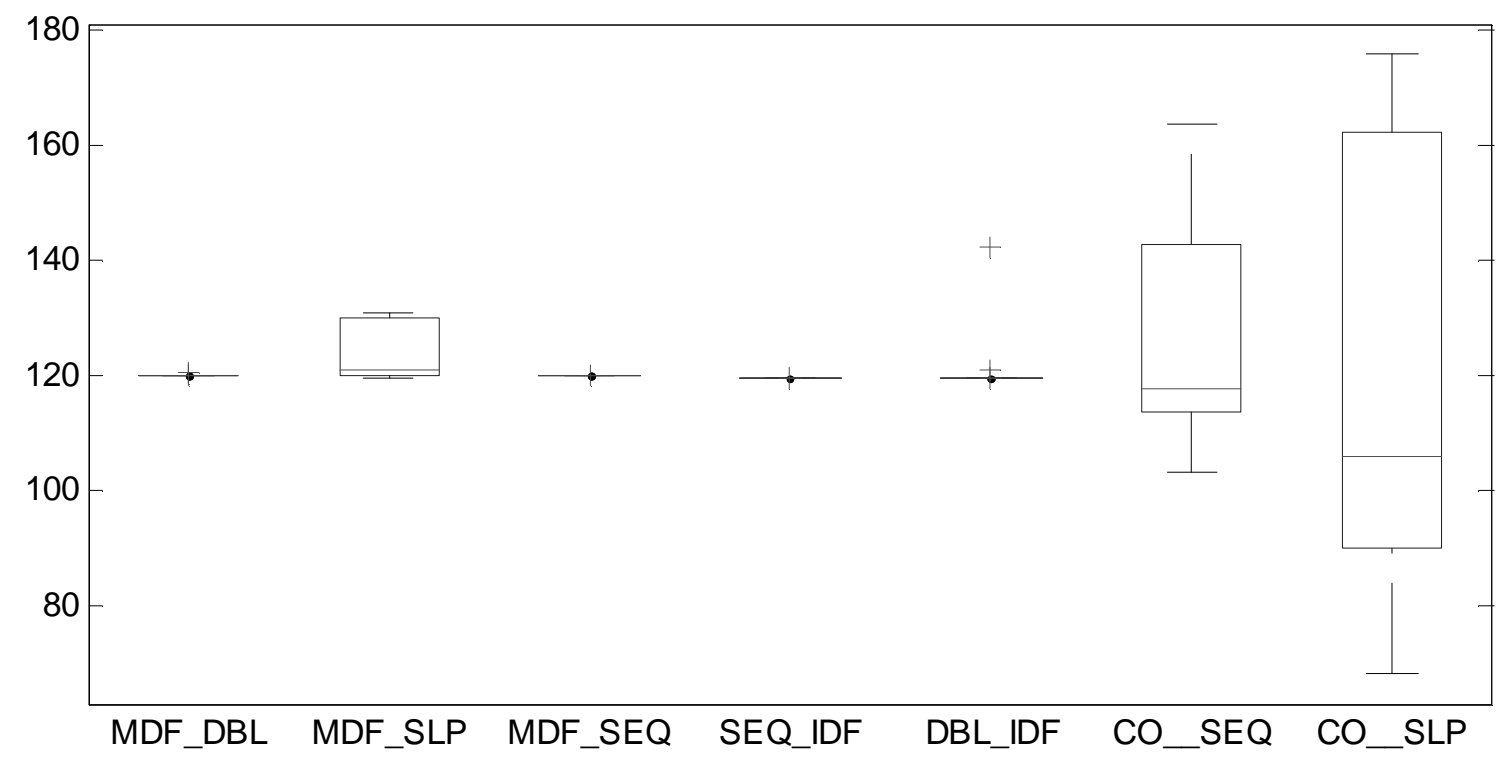

Figure 3.2.4: Solution Distribution Box Plot

dian observed values are indicated by the horizontal line. The box indicates the size of the upper and lower quartile of the solutions for each method. Outliers are indicated by the '+' symbol. The exact solution to the FORM for $3 \sigma$ is 119.68. The MDF/DBL/PMA, $\mathrm{MDF} / \mathrm{SEQ} / \mathrm{PMA}$, and the SEQ/IDF/PMA methods were observed to be very accurate with relatively few outliers in the predicted optimum point. The single-loop based methods produced some scatter and a distinct bias from the exact solution. This is consistent with the results obtained from the single-loop example in Section 3.2.1. This scatter clearly led to convergence problems for the single-loop approach in a $\mathrm{CO}$ framework. Both CO-based algorithms incurred a number of failed runs, as shown in Table 3.6. The sequential IDF method exhibited a clear superiority in efficiency and accuracy for the analytical optimization. The MDF-based methods were stable and relatively accurate, but required an order of magnitude more function evaluations than the IDF based methods to solve. The CO based methods produced the most scatter in the location of the optimum point. 
Table 3.6: Algorithm Stability Comparison

\begin{tabular}{llll}
\hline method & number of & \multicolumn{2}{c}{ avg function evals } \\
& failed runs & discipline 1 & discipline 2 \\
\hline MDF/DBL/PMA & 0 & 16998 & 16998 \\
MDF/SLP & 1 & 10128 & 10128 \\
MDF/SEQ/PMA & 0 & 7440 & 7440 \\
IDF/SEQ/PMA & 0 & 1229 & 1229 \\
IDF/DBL/PMA & 2 & 8489 & 8489 \\
CO/SEQ/PMA & 16 & 57105 & 135190 \\
CO/SLP & 10 & 7427 & 16005 \\
\hline
\end{tabular}

\subsubsection{Multi-Discipline Truss Example Optimization}

A multi-disciplinary truss optimization problem was developed to test the RBDO-MDO strategies on a less well-behaved problem than the analytical example. Additionally, the problem was designed to be a better test-bed for the $\mathrm{CO}$ based RBDO solver. The truss structure consists of two sub-structures: a span and a support. The two sub-structures can be considered as distinct disciplines coupled by the reaction force and displacement at the location of the interface between the support and the span. The full structure and the two sub-structures are shown in Figure 3.2.5.

The design variable and coupling variable assignments are shown in Table 3.7. The two sub-structures can be considered independently. They can be isolated into separate disciplines with no shared variables. The FEM analysis output - the reaction force and displacement at the interface node - for each structure is required by the other to solve. This required the introduction of two coupling variables - the reaction force and vertical displacement at the interface node. The problem formulations for the MDF and IDF methods are straightforward. The design variables are all solved simultaneously with one system optimizer. The IDF method introduces the displacement and reaction force variables into the system optimizer. The $\mathrm{CO}$ formulation isolates all the variables pertaining to each sub-structure into their respective disciplines. There are no shared design variables, so the 


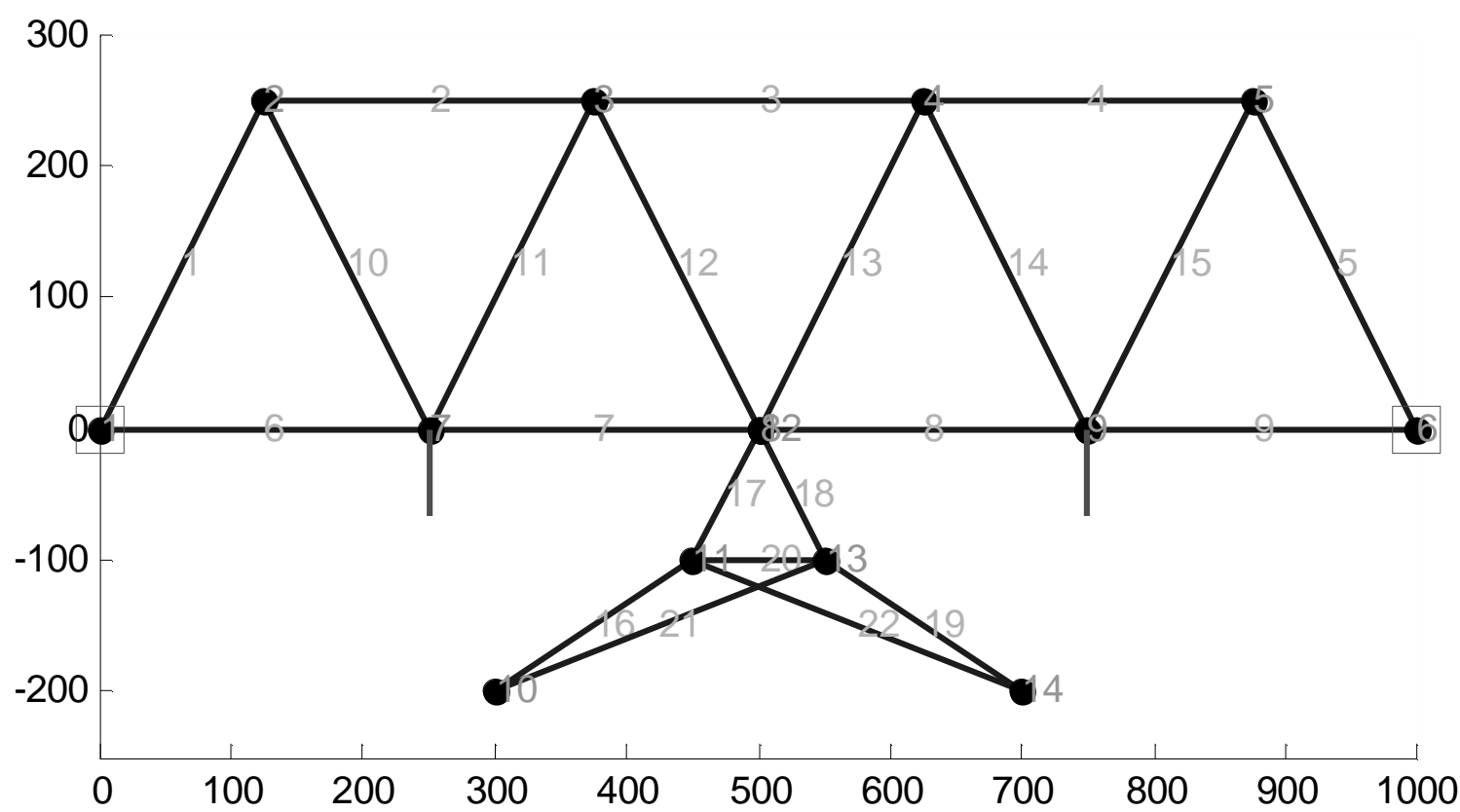

(a) Full truss

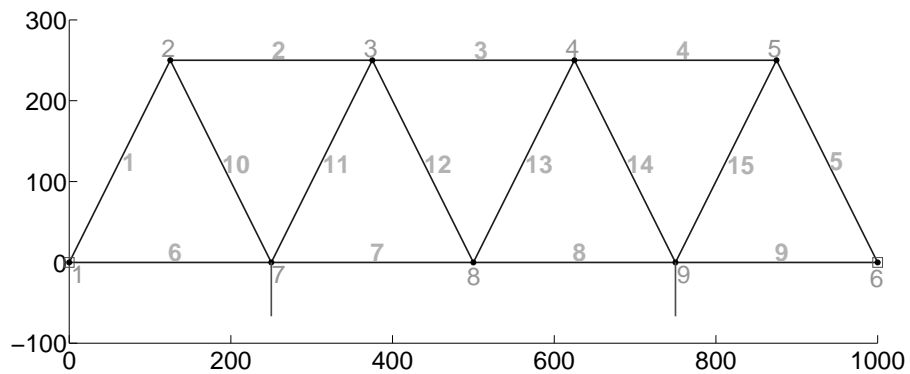

(b) Sub-part 1

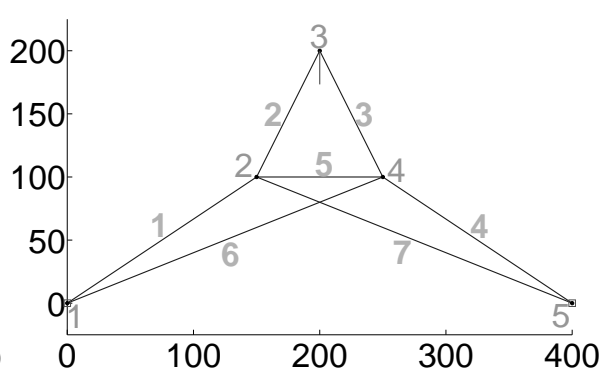

(c) Sub-part 2

Figure 3.2.5: Decoupled Structure

system optimization is a function of only the reaction force and displacement. The individual disciplines are responsible to provide an optimum structure for the target displacement and reaction force set by the system optimizer. The $\mathrm{CO}$ formulation is shown in equation 3.2.5, where $m_{1,2}^{*}$ represents the optimum mass values returned by the local discipline optimization problems. The variables $U$ and $R$ represent the displacement and reaction force at the interface node respectively. The ultimate stress is denoted by $\sigma_{\max }$, which was assumed 
to be uncertain with a mean value of 20000 psi and a standard deviation of 2000 psi.

$$
\begin{gathered}
\min \underset{U_{\text {sys }}, R_{\text {sys }}}{f}=m_{1}^{*}\left(U_{\text {sys }}, R_{\text {sys }}, \bar{\sigma}_{\text {max }}\right)+m_{2}^{*}\left(U_{\text {sys }}, R_{\text {sys }}, \bar{\sigma}_{\text {max }}\right) \\
\text { s.t. } \sum_{i=1,2}\left(U_{\text {sys }}-U_{i}\right)^{2}+\left(R_{\text {sys }}-R_{i}\right)^{2}=0 \\
A_{i=1,2=\min (}\left(U_{\text {sys }}-U_{i}\left(x_{i}, \sigma_{\text {max }}\right)\right)^{2}+\left(R_{\text {sys }}-R_{i}\left(x_{i}, \sigma_{\text {max }}\right)\right)^{2} \\
\text { s.t. } g_{i}=P\left(\sigma \leq \sigma_{\text {max }}\right) \geq P_{\text {goal }} \\
\sigma_{\max } \sim N(20000,2000) \text { psi }
\end{gathered}
$$

Table 3.7: MDO Truss Design Variables

\begin{tabular}{clll}
\hline variable & definition & & \\
\hline var & discipline 1 & var & discipline 2 \\
$x_{1}$ & $A_{1}, A_{2}, A_{3}, A_{4}, A_{5}$ & $x_{1}$ & $A_{1}, A_{2}, A_{3}, A_{4}$ \\
$x_{2}$ & $A_{6}, A_{7}, A_{8}, A_{9}$ & $x_{2}$ & $A_{5}, A_{6}, A_{7}$ \\
$x_{3}$ & $A_{10}, A_{11}, A_{12}, A_{13}, A_{14}, A_{15}$ & $x_{3}$ & $X_{2}, X_{4}$ \\
$x_{4}$ & $X_{2}, X_{5}$ & $x_{4}$ & $Y_{2}, Y_{4}$ \\
$x_{5}$ & $Y_{2}, Y_{5}$ & & coupling \\
$x_{6}$ & $X_{3}, X_{4}$ & $Y_{1}$ & $\mathrm{R}$ \\
$x_{7}$ & $Y_{3}, Y_{4}$ & $Y_{2}$ & $\mathrm{U}$ \\
\hline
\end{tabular}

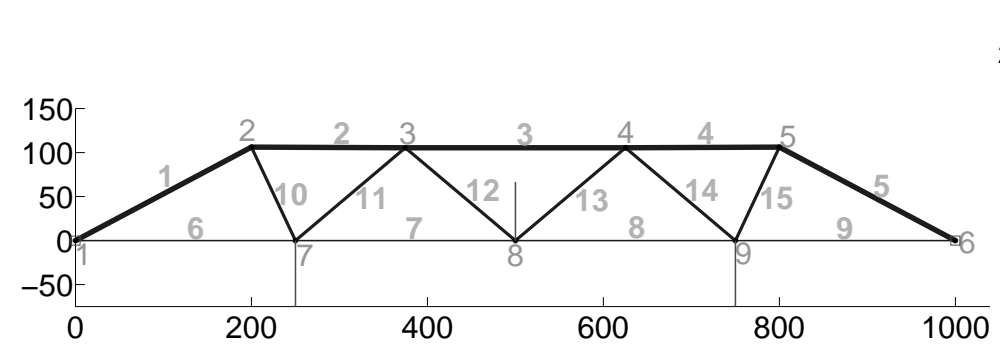

(a) Discipline 1

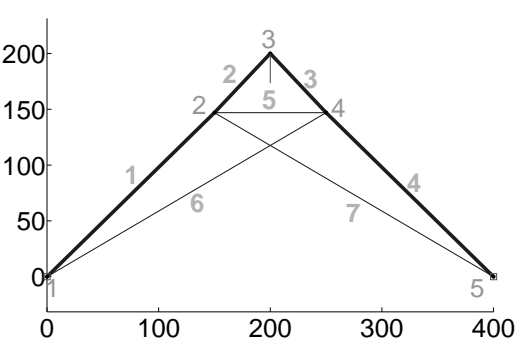

(b) Discipline 2

Figure 3.2.6: Optimized Truss

The results are shown in Table 3.8. The deterministic solution has a mass of under 400 
lb. Considering the uncertainty at a reliability level of $6 \sigma$ increases the optimum mass to approximately $1000 \mathrm{lb}$ for all methods considered. As before, the IDF methods were found to be more efficient than the MDF-based approaches, with the sequential IDF method having the fewest function evaluations for discipline 1. However, the CO/Sequential method required significantly fewer function evaluations in the second discipline, and was the most efficient approach for the MDO truss example. However, multiple starting points had to be attempted before the solution was in agreement with the other methods, and the compatibility constraints had to be relaxed to obtain a solution at all.

Table 3.8: MDO Truss Example Solution

\begin{tabular}{lccc}
\hline $6 \sigma$ Solution & mass & \multicolumn{2}{c}{$n_{\text {evals }}$} \\
& & Dis 1 & Dis 2 \\
\hline Deterministic & 369.2 & 7204 & 7204 \\
MDF/SEQ/PMA & 950.6 & 366266 & 366266 \\
MDF/SLP & 1007.1 & 62392 & 62392 \\
MDF/DBL/PMA & 994.1 & 92448 & 92448 \\
IDF/DBL/PMA & 987.6 & 45117 & 45117 \\
IDF/SEQ/PMA & 954.2 & 32151 & 32151 \\
CO/SEQ/PMA & 996.7 & 36786 & 15301 \\
\hline
\end{tabular}

\subsection{Summary}

The development of RyeMDO, an optimization package for multi-objective, multi-disciplinary RBDO, was described. Several modules for reliability assessment, RBDO, and MDO were developed. The algorithms were validated by solving several well-known analytical RBDO test problems and truss optimization problems for several combinations of reliability assessments, optimization strategies, and MDO methods.

The performance characteristics of FORM-based reliability assessment methods were com- 
pared using both an analytical problem and an 18 bar truss optimization problem. It was found that the single loop approach was significantly more efficient than the exact approaches, but diverged slightly in accuracy with increasing reliability levels. Of the FORM based methods, the sequential approach was observed to be the most efficient for both problems studied, and was found to reliably converge to the solution from almost any attempted starting vector.

The sequential and double loop approaches were found to exhibit the best accuracy in the analytical problems, where the true exact solutions were known. The sequential methods performed better than the double loop approach in both the MDF, IDF, and CO MDO frameworks. The single-loop/MDF method was found to be efficient, but exhibited bias in both the single discipline examples and the multi-discipline examples. Additionally, when tested from many starting vectors, the solutions obtained with the single-loop/MDF and the single-loop/CO methods were found to be prone to scatter due to the premature convergence of some of the runs. It was found that difficulties arise when gradient based optimizers are used with the $\mathrm{CO}$ architecture. Since the reliability analysis requires the numerical solution of an inner optimization loop for every constraint, numerical errors can build up. This is problematic when a gradient based system optimizer attempts to compute accurate gradients by the finite-differencing of the target values for the local discipline optimizations. The IDF/Sequential approach using PMA based reliability assessment was found to be consistently efficient and accurate. Chapter 4 utilizes several of the RBDO-MDO methods available in RyeMDO to solve a practical engineering problem: the conceptual design optimization of an aircraft wing box structure with an uncertain contributing analysis. 


\section{Chapter 4}

\section{Aircraft Wing Box Conceptual Design Considering Model Uncertainty}

This chapter studies the conceptual design optimization of an aircraft wing box structure. It was developed as a proof-of-concept case study of the database-driven approach for quantifying probabilistic error terms resulting from uncertain analysis methods. In the case study, a surrogate model was developed from a sample of FEM analyses and was considered as an uncertain analysis method. The surrogate model served as a method for estimating the maximum stress in the wing box. A database containing finite element solutions for many designs was created and used to assess the relative error between the surrogate model and the FEM solver. The advantage of such a case study is the ability to back check any optimized solution attained using the low fidelity model with high fidelity analysis. This ability is not available for the aircraft conceptual design case study, which relies on a historical database of currently available aircraft designs. The case study serves as a proof of concept for the proposed methodology, which is carried forward in an aircraft conceptual design optimization case study in chapter 5 . 
Practical engineering problems are often characterized by computationally costly simulation or high fidelity analysis methods such as CFD or FEM. The design optimization of such systems can be very computationally expensive since many function evaluations may be required to obtain a converged solution. Additionally, non-smooth objective or constraint functions may hamper the convergence of gradient based optimization methods. Surrogate modeling is a procedure for reducing computational cost in optimization by representing the high fidelity analysis methods mathematically. The high fidelity methods are sampled across a predetermined set of design variables. The surrogate models utilize the sample data to mathematically represent the design space. Optimization solutions can be rapidly obtained. For these reasons, surrogate models are widely implemented in design optimization [132]. However, surrogate models only approximate the true system equations, and therefore introduce uncertainty. The optimum designs obtained using surrogate models may be found to be infeasible when the design is subjected to high fidelity analysis methods. RBDO can be used to manage the uncertainties introduced by surrogate models in order to increase the confidence a designer may place in an optimization solution obtained using surrogate models.

A parametrized finite-element model of a generic light business jet wing box was developed and is shown in Figure 4.0.1. The aircraft concept considered was similar in size and performance to currently available light jets such as the Cessna Mustang or the Diamond Jet. Following common practice in aircraft conceptual design, a target aircraft mass and a wing group weight budget was assigned. The performance targets of the conceptual wing design were selected to match values typical to small light jet aircraft. The target gross aircraft mass was assumed to be $5200 \mathrm{~kg}$ with a wing-stored fuel capacity of $1200 \mathrm{~kg}$. The wing weight budget was assumed to be $440 \mathrm{~kg}$ for the load bearing structure. The maximum von Mises stress was constrained to be below $360 \mathrm{MPa}$, corresponding to the yield strength 
of aluminum 7075 with a safety margin of 1.5 as required by airworthiness standards. The optimization objective was to maximize the wing lift-to-drag ratio at a cruise speed of 400 kts and an altitude of $10670 \mathrm{~m}$ (35000 ft). The body contribution to the lift-to-drag ratio was neglected. The FEM analysis was replaced by a Kriging surrogate model. Kriging models have been shown to provide good approximations of non-linear functions relative to other approaches [28, 133, 134]. Each FEM based function evaluation required approximately 60 seconds on a desktop computer. Evaluating the surrogate model required much less than one second. An uncertain error term was introduced to account for discrepancies between the stress estimated using the approximation model and the stress calculated by FEM.
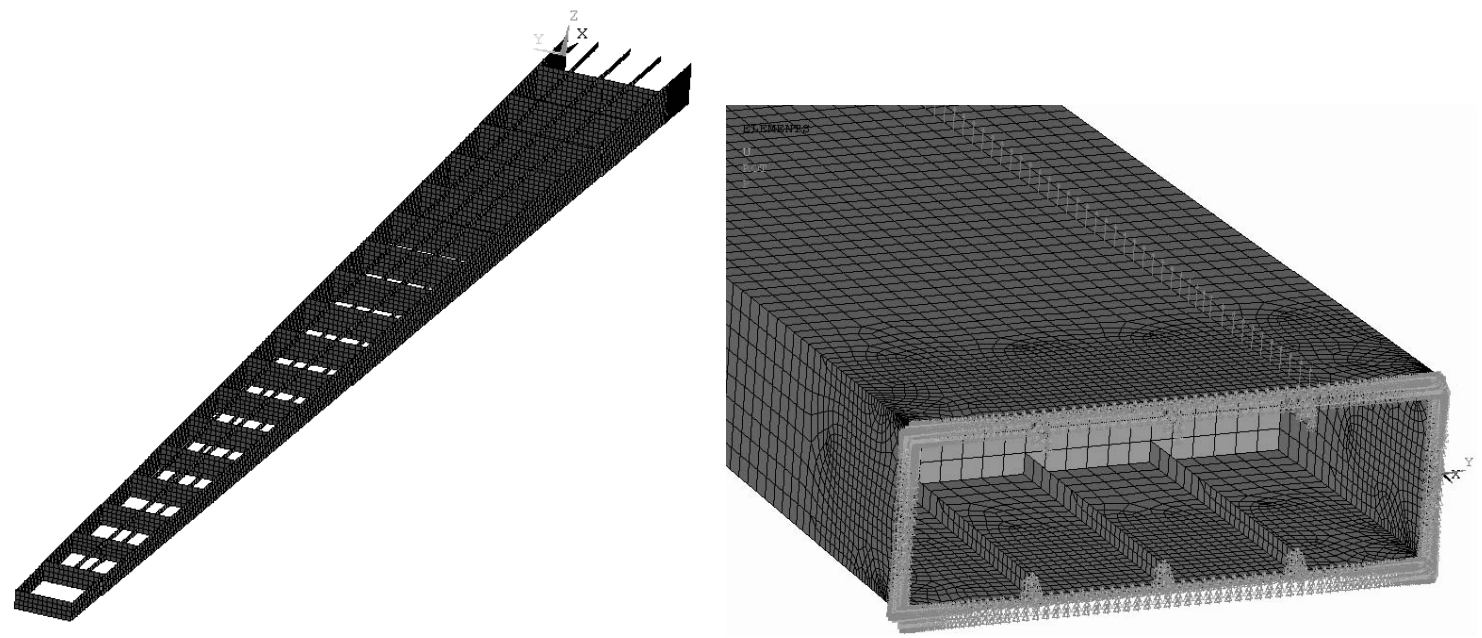

Figure 4.0.1: Wing Box FEM Model

\subsection{Problem Description}

The problem was formulated as a multi-discipline optimization with two contributing analysis methods: a vortex-lattice aerodynamics solver and a structures solver consisting of a Kriging approximation model. The approximation model was generated using a database of finite-element solutions sampled evenly across the design space. This model was con- 
sidered as an uncertain contributing analysis by obtaining the probability distribution of the model error between FEM runs and the surrogate model.

The FEM model consists of 29 member attributes representing the thicknesses of the primary structural members - 19 ribs, the front and rear spar, 6 stringers, and the upper and lower skin. The dimensionality was reduced by linking the attributes to 7 design variables as shown in Table 4.1 and Figure 4.1.1. Two variables were introduced to alter the overall wing geometry: span and wing reference area, making a total of 9 design variables. The sweep angle, taper ratio, and airfoil shape were held constant. Considering all 29 attributes additional wing shape variables would potentially yield better designs. However, the accuracy of the surrogate models with 31 dimensions was found to be extremely low.

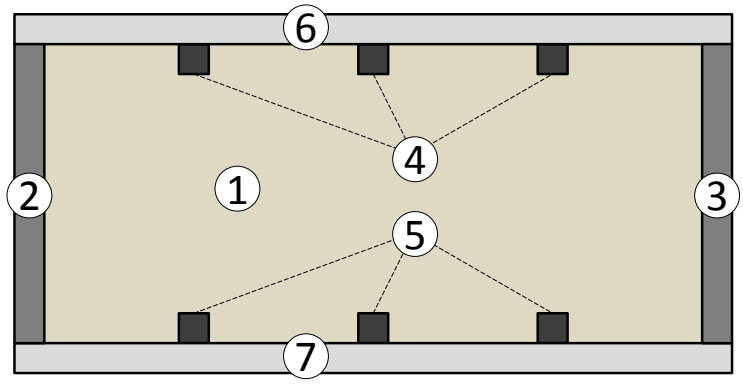

Figure 4.1.1: Structural Discipline Variables

\subsection{Surrogate Model}

Kriging models are widely used as surrogates for computationally expensive analysis methods in design optimization [135, 136]. Unlike response surface models, Kriging models always pass through the supplied design points. A database of finite element analysis solutions was sampled across 200 evenly distributed design points to build a second order Kriging surrogate model, which was considered as an uncertain analysis method. Increasing the database beyond 200 did not appreciably improve the accuracy of the approxima- 
tion model. An error term, defined in equation 4.2.1, was introduced, representing the ratio of the model predicted maximum von-Mises stress to the stress calculated by a Finite Element Method (FEM) based analysis using ANSYS, a well known commercial finite element solver. The error term is denoted by $\varepsilon_{\sigma}$ and the model predicted stress and FEM stress values are denoted by $\sigma_{p}$ and $\sigma_{F E M}$ respectively.

$$
\varepsilon_{\sigma}=\sigma_{p} / \sigma_{F E M}
$$

\subsection{Model Error}

The Kriging model was considered as a 'black box' analysis module. The error associated with the model was represented probabilistically. The PDF was estimated by sampling the approximation model and the FEM model with 200 random, uniformly distributed design variable vectors. This data is shown in Table A.3 in the appendix. The ratio of the maximum stress predicted by the model and that obtained by the FEM analysis for each of the 200 random designs was stored. A histogram of the obtained error ratios was found to approximate a normal distribution curve with a mean value of 1 . Increasing the sample size beyond 200 did not significantly change either the distribution shape, the mean, or the standard deviation. The histogram of the error ratio term and the estimated PDF is shown in Figure 4.3.1.

\subsection{Solution Strategy}

The wing box was optimized with RyeMDO using both the MDF and CO modules and the double loop, sequential, and single loop modules. The problem formulation using the 


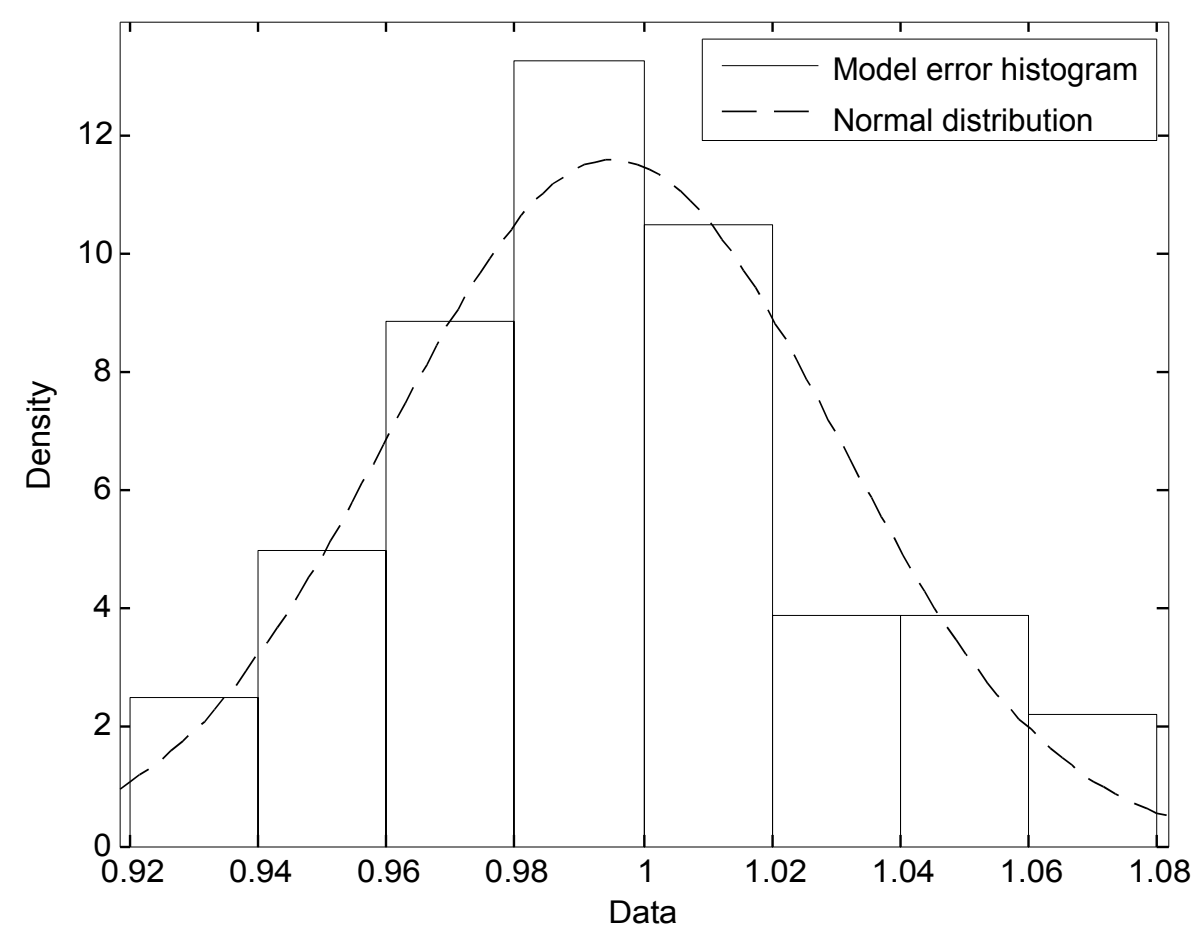

Figure 4.3.1: Kriging Model Error Distribution: $\mu=1.0039, \sigma=0.0736$

MDF based methods is shown in equation 4.4.1 where $L / D$ is the wing lift-to-drag ratio, and $b$ and $S$ denote the wing span and wing reference area respectively. Structural thickness values are denoted by $t$. The constraints are given in Table 4.2 .

$$
\begin{aligned}
\max \frac{L}{D} & =f\left(b, S, t_{1 \ldots 7}\right) \\
P\left(\sigma \leq \sigma_{\max }\right) & \geq P_{\text {goal }} \\
M & \leq M_{\text {goal }} \\
V_{a} & \leq V_{\text {a,max }}
\end{aligned}
$$

The objective function was defined to maximize the wing $L / D$. The constraints were as follows: the mass $M$, and the approach speed $V_{a}$, must be less than the limits and the probability that the stress $\sigma$, is less than the limit stress is greater than the target probability. 
Note that every function and constraint evaluation is an MDA loop to ensure compatibility between the disciplines. The solution strategy for the $\mathrm{CO}$ architecture is shown in Figure 4.4.1. The problem is divided into aerodynamics and structures sub-problems. The aerodynamics sub-problem is a function of only the wing span and wing area design variables since the thickness values of the structural members are not required for the aerodynamics discipline to evaluate. The structures discipline depends on both the member thicknesses and the overall wing shape, and is therefore a function of all of the 9 design variables considered. The system level problem was defined as a function of the two common variables - wing span and area. Auxiliary constraints $A_{\text {aero }}$ and $A_{\text {struct }}$ force consistency between the disciplines. Any single-discipline deterministic or RBDO approach can be used for the local optimizations.

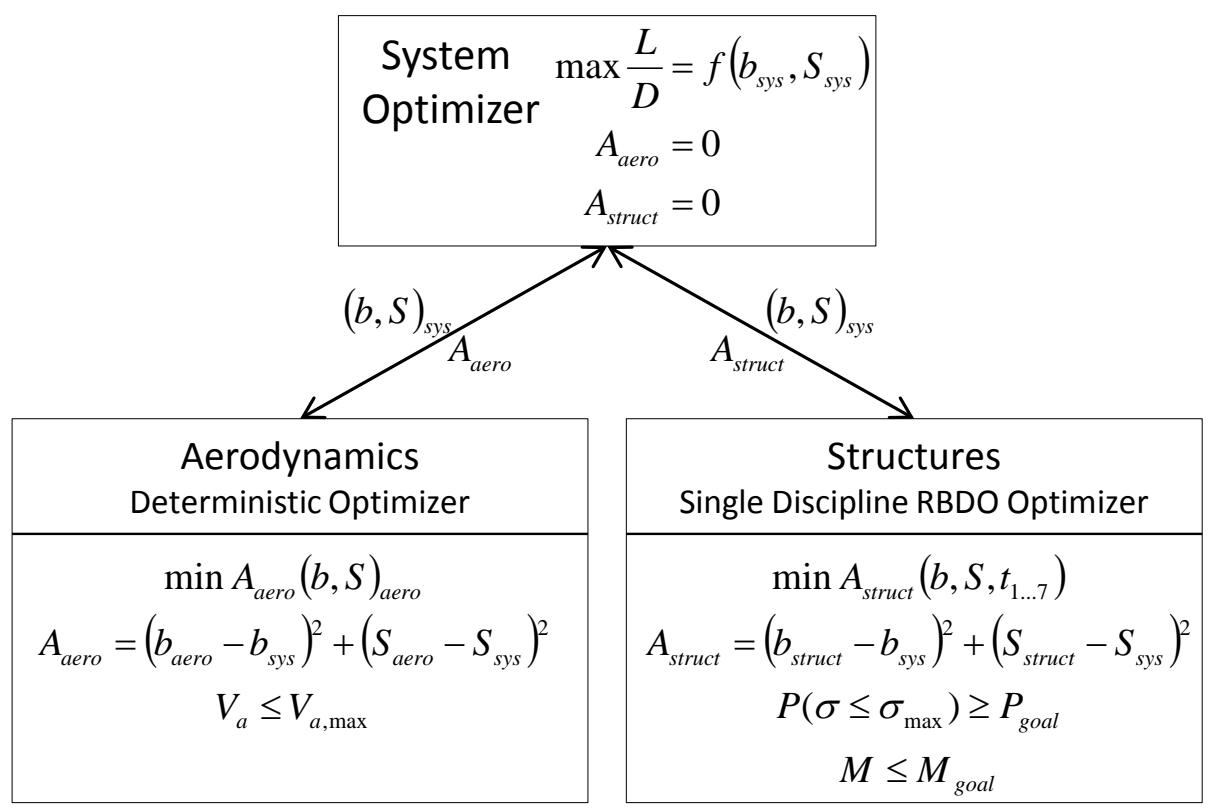

Figure 4.4.1: Collaborative Optimization with RBDO 


\subsection{Results}

The optimization was solved for reliability levels of 0 to 6 , corresponding to failure probabilities of $50 \%$ to approximately $10^{-7} \%$, using each RBDO-MDO approach. Figure 4.5.1 shows the influence of increasing target reliability indices on the location of the optimum. The predicted values of the maximum stress at the optimum point for each target reliability level lie on the stress constraint boundary when $\beta$ is zero. When $\beta$ increases, the wing shape and material thicknesses are altered, as shown in Table 4.3, such that the predicted stress moves farther into feasible design space. The predicted optimum points were evaluated using FEM analysis to check for possible discrepancies between the stress predictions and the actual stress. The FEM derived stress values are larger than the predicted stress, indicating that the structure designed using the low-fidelity approach fails when subjected to better analysis. For reliability indices larger than $\beta=1.2(88 \%)$, the design is feasible. Using the standard $3 \sigma$ or $6 \sigma$ reliability levels yields conservative designs that fall well within the feasible region when subjected to the high fidelity analysis.

Figure 4.5.2 shows the influence of the reliability index on the shape of the wing. The wing shape becomes more conservative as the reliability index increases. The deterministic optimum wing shape has the largest aspect ratio (a measure of the slenderness of the wing). Wings having larger aspect ratios generally exhibit lower induced drag. However, a slender wing requires increased structural member thicknesses to maintain strength. Increases to the reliability level increases the margin by which the designs must exceed the specified stress constraint while remaining under the mass budget. The reliable wing designs trade aerodynamic efficiency for structural efficiency by reducing the slenderness of the wing while remaining at or below the prescribed weight budget. Table 4.3 indicates that increases to the reliability index has a slight influence on the thicknesses of structural members and a major influence on the wing planform shape. 

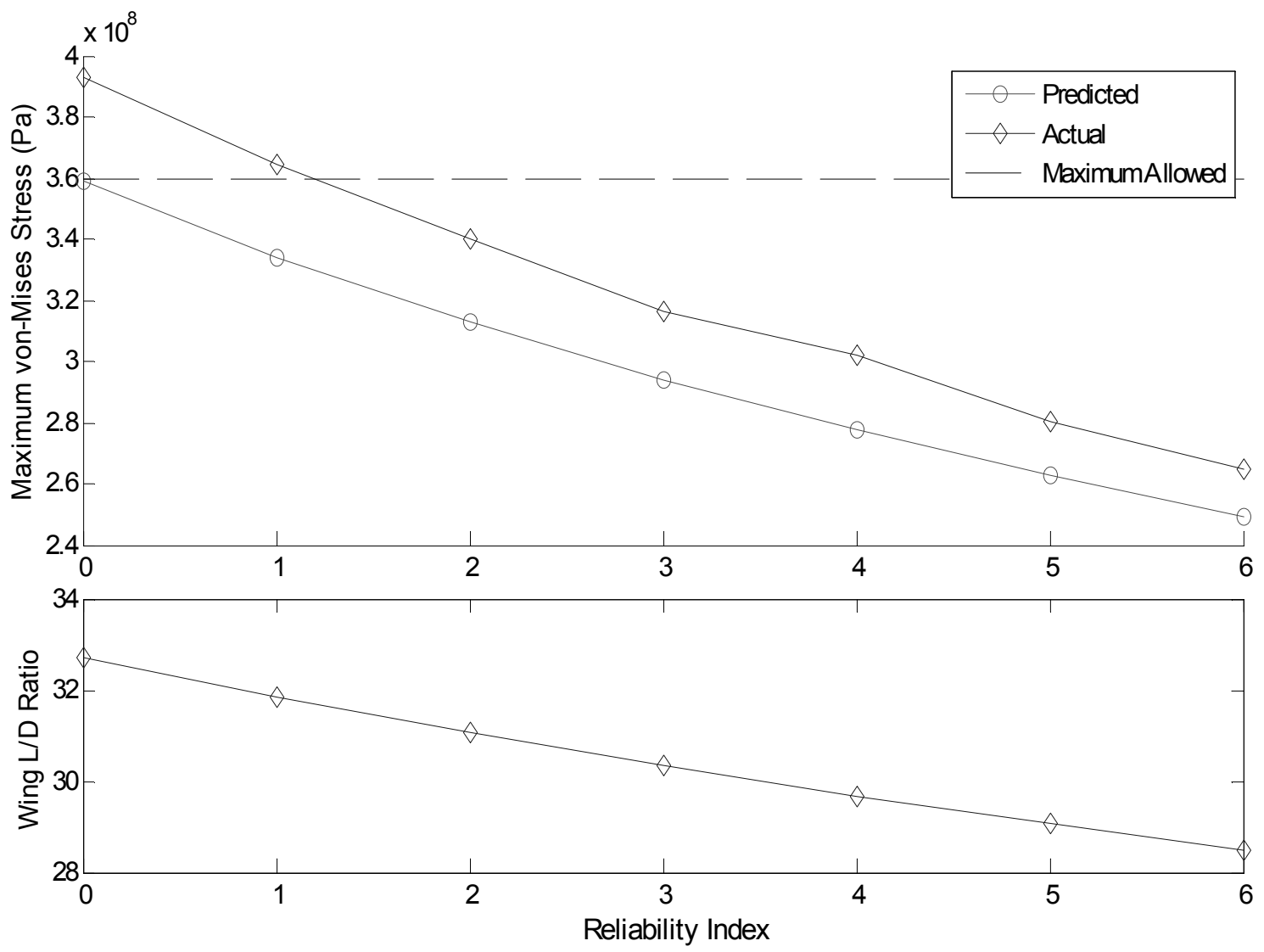

Figure 4.5.1: Wing Box RBDO Results

\subsection{Algorithm Performance Comparison}

Each RBDO-MDO architecture considered in the case study was run with a reliability level of 6 to compare the relative performance of each approach. As shown in Table 4.4, the single loop methods have significant performance advantages, but predict optimum L/D ratios that are slight outliers from the methods that use exact solutions to FORM. The CO based approaches require many structural function evaluations but relatively few aerodynamics evaluations due to the reduced dimensionality of the 2-variable aerodynamics sub problem. As a consequence, despite requiring many evaluations of the structures discipline, the solution times for the $\mathrm{CO}$ methods were found to be competitive since evaluating the structures 


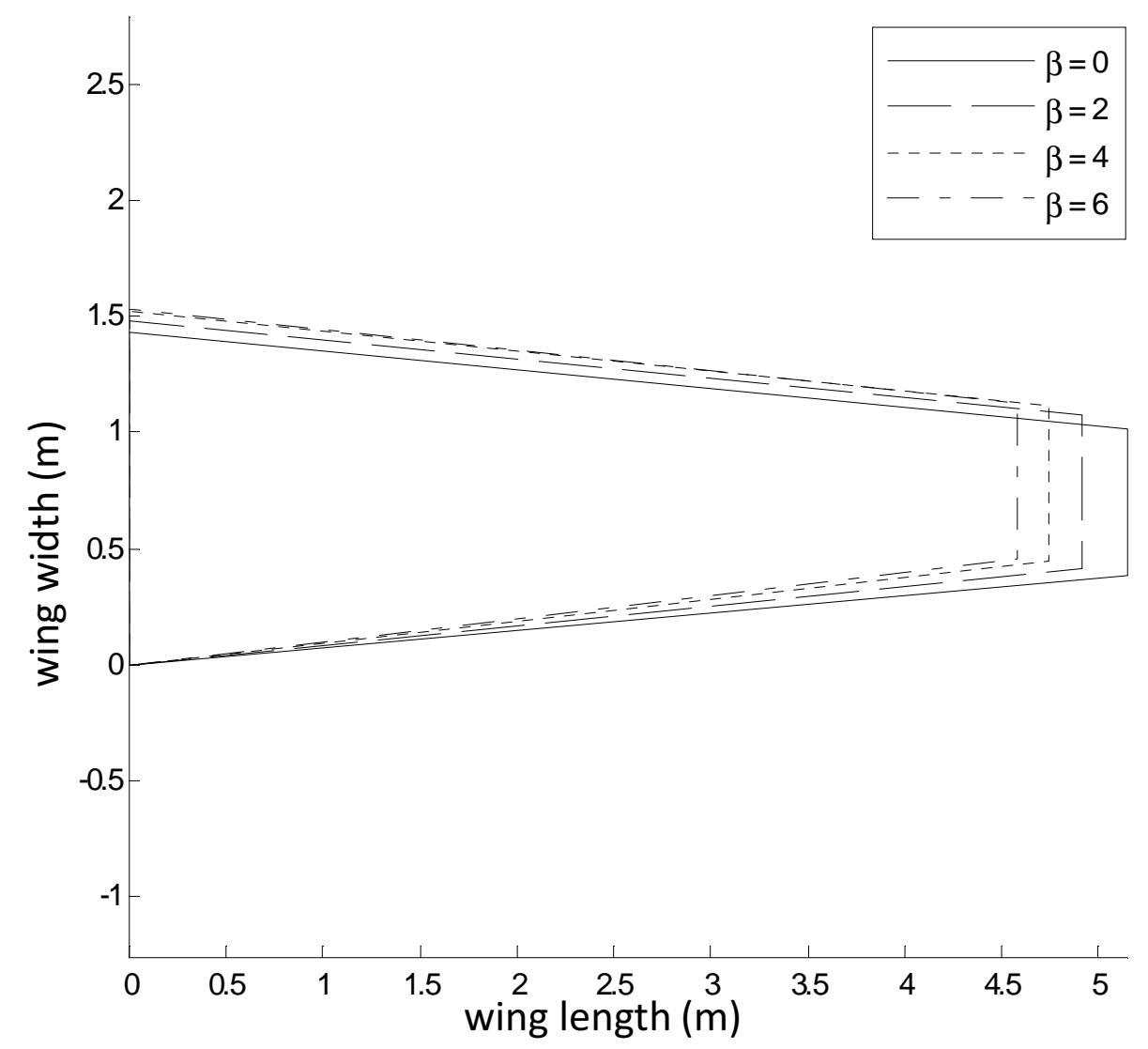

Figure 4.5.2: Wing Planform 
approximation model is extremely fast compared to calls to the aerodynamics solver.

\subsection{Summary}

The wing box of a generic light jet similar to the Cessna Mustang was optimized using RyeMDO while considering the model uncertainty introduced by a surrogate model. The error distributions were evaluated by uniformly sampling the Kriging models and comparing the predicted maximum stress with FEM solutions. The solution indicates that under traditional optimization, the structure optimized with an approximation model violates the stress constraint when subjected to high fidelity analysis. However, it was shown that RBDO with a sufficiently low failure probability protects the low fidelity solution from producing infeasible designs. Additionally, the performance of each RBDO-MDO architecture was evaluated and compared. The single loop approaches were the most efficient for both the MDF and $\mathrm{CO}$ methods. However, the solutions deviated slightly from the methods that employ exact solutions to FORM, indicating a slight loss in accuracy. The CO based method with sequential RBDO significantly reduced the number of aerodynamic discipline evaluations by restricting local structural variables to the local structural optimization problem. Since the model evaluations are very fast relative to the aerodynamics solver, the $\mathrm{CO} /$ Sequential method exhibited solution times that were competitive with the other approaches despite the large number of structural discipline evaluations. This indicates that the $\mathrm{CO}$ method can be advantageous if costly analyses can be isolated into a sub-problem with low dimensionality. However, for problems having few local variables and high dimensionality in all sub-problems, $\mathrm{CO}$ is unlikely to provide any advantages, and is likely to be outperformed by the single-level MDO approaches. The wing box example indicates how uncertainties associated with approximate contributing analyses in the form 
of surrogate models can be managed using RyeMDO. Chapter 5 shows how the errors associated with traditional low fidelity aircraft conceptual design methodology can be managed using RyeMDO. 
Table 4.1: Member Attribute List

\begin{tabular}{|c|c|c|cc|}
\hline & Member & Variable & \multicolumn{2}{|c|}{ Limit (mm) } \\
& & Number & lower & upper \\
\hline 1 & rib 1 & & & \\
2 & rib 2 & & & \\
3 & rib 3 & & & \\
4 & rib 4 & & & \\
5 & rib 5 & & & \\
6 & rib 6 & & & \\
7 & rib 7 & & & \\
8 & rib 8 & & & \\
9 & rib 9 & & & \\
10 & rib 10 & 1 & 2 & \\
11 & rib 11 & & & \\
12 & rib 12 & & & \\
13 & rib 13 & & & \\
14 & rib 14 & & & \\
15 & rib 15 & & & \\
16 & rib 16 & & & \\
17 & rib 17 & & & \\
18 & rib 18 & & & \\
19 & rib 19 & & & \\
\hline 20 & front spar & 2 & 10 & 30 \\
\hline 21 & rear spar & 3 & 10 & 30 \\
\hline 22 & upper stringer 1 & & & \\
23 & upper stringer 2 & 4 & 2 & \\
24 & upper stringer 3 & & & \\
\hline 25 & lower stringer 1 & & & \\
26 & lower stringer 2 & 5 & 2 & \\
27 & lower stringer 3 & & & \\
\hline 28 & upper skin & 6 & 15 & 30 \\
\hline 29 & lower skin & 7 & 15 & 30 \\
\hline & & & & \\
\hline
\end{tabular}

Table 4.2: Constraints

\begin{tabular}{lll}
\hline constraint & symbol & value \\
\hline maximum stress & $\sigma$ & $360 \mathrm{Mpa}$ \\
mass & $M$ & $440 \mathrm{~kg}$ \\
approach speed & $V_{a}$ & $120 \mathrm{kts}$ \\
\hline
\end{tabular}


Table 4.3: Design Variable Values - MDF/Sequential Method

\begin{tabular}{ccccccccccc}
\hline$\beta$ & A1 & A2 & A3 & A4 & A5 & A6 & A7 & $b$ & $S$ & $L$ \\
- & $m m$ & $m m$ & $m m$ & $m m$ & $m m$ & $m m$ & $m m$ & $m$ & $m^{2}$ & - \\
\hline 0 & 2.30 & 18.1 & 15.2 & 4.71 & 6.02 & 18.4 & 14.9 & 10.3 & 10.6 & 31.9 \\
1 & 2.30 & 17.9 & 15.2 & 4.68 & 5.92 & 18.5 & 15.0 & 10.0 & 10.6 & 31.1 \\
2 & 2.30 & 17.9 & 15.5 & 4.66 & 5.91 & 18.5 & 15.2 & 9.83 & 10.5 & 30.9 \\
3 & 2.30 & 17.9 & 15.7 & 4.57 & 5.81 & 18.5 & 15.5 & 9.64 & 10.4 & 30.3 \\
4 & 2.30 & 17.8 & 15.8 & 4.56 & 5.80 & 18.6 & 15.5 & 9.48 & 10.4 & 29.7 \\
5 & 2.30 & 17.8 & 16.1 & 4.47 & 5.71 & 18.8 & 16.0 & 9.30 & 10.2 & 29.1 \\
6 & 2.30 & 17.8 & 16.5 & 4.45 & 5.70 & 18.8 & 16.2 & 9.16 & 10.1 & 28.5 \\
\hline
\end{tabular}

Table 4.4: RBDO-MDO Performance Comparison

\begin{tabular}{llll}
\hline & \multicolumn{2}{l}{ function calls } & objective \\
& Aero & Struct & L/D \\
\hline MDF/Sequential & 96 & 96 & 28.50 \\
MDF/Double Loop & 105 & 105 & 28.49 \\
MDF/Single Loop & 37 & 37 & 28.22 \\
CO/Sequential & 42 & 2362 & 28.61 \\
CO/Double Loop & 87 & 4931 & 28.60 \\
CO/Single Loop & 44 & 985 & 28.07 \\
\hline
\end{tabular}




\section{Chapter 5}

\section{Aircraft Conceptual Design Considering Uncertain Contributing Analysis Methods}

This chapter proposes a novel approach for improving aircraft conceptual design optimization. Multi-disciplinary RBDO algorithms were implemented to manage the errors associated with the traditional low fidelity analysis used in aircraft conceptual design. Probabilistic models of the errors associated with each implementation of empirical equations were developed by comparing the analysis output with historical data derived from a specification database of currently available aircraft designs. These error terms were introduced into the optimization problem, allowing the errors to propagate their influence on each constraint. The approach allows designers to constrain the failure risks associated with the approximate analysis methods to an allowable level. This reduces the likelihood that a conceptual design optimized with low fidelity analysis methods will fail to meet the design goals when subjected to more detailed analysis later in the design process, which can 
lead to time consuming and costly design revisions. RBDO targets only active constraints, adjusting designs away from active constraint boundaries within the optimization scheme. Designs are therefore only just as conservative as they need to be to reach a specified failure probability.

The approach developed in this research uses non-empirical analysis where possible. Low fidelity equations are used where the available non-empirical analysis methods are too computationally expensive to implement at the conceptual design level. The errors introduced by the low fidelity equations were quantified using information derived from an aircraft specification database. The database compiled for this research is shown in Table A.1. The database contains the published dimensions and performance of some currently available twin-engine commercial passenger aircraft. Each design in the database was modeled and subjected to the discipline analysis packages (aerodynamics, structures, and performance) to calculate predicted performance and mass characteristics for each design. The errors between the predicted characteristics and the performance indicated in the aircraft database were considered as uncertain parameters in the optimization scheme.

Enhanced RBDO algorithms are proposed for determining the influence of increasing the target reliability index on the optimized aircraft designs. The reliability index was introduced as a second objective function, and the optimization was solved with several of the most promising RBDO methods using RyeMDO.

\subsection{Problem Description}

The conceptual design of a regional commercial aircraft similar to the Airbus A320-200 and the Boeing 737-800 was considered. The basic design goals, shown in Table 5.1, were set to equal or exceed that of the 737-800 for comparison. 
Table 5.1: Design Goals

\begin{tabular}{ll}
\hline goal name & target value \\
\hline passengers (economy/business) & $N_{p a x}=160 / 16$ \\
payload mass & $M_{p l}=21300 \mathrm{~kg}$ \\
range (200 km reserve) & $R \geq 5670 \mathrm{~km}$ \\
cruise mach & $M_{c r}=0.80$ \\
cruise altitude & $h_{c r}=10671 \mathrm{~m}$ \\
takeoff distance & $S_{t o} \leq 2400 \mathrm{~m}$ \\
approach speed & $V_{a} \leq 140 \mathrm{kts}$ \\
\hline
\end{tabular}

An algorithm was developed that establishes the basic layout of the design given the specified design goals and the current design variables to develop a model that describes the aircraft geometry with sufficient detail for the various analysis methods to run. The analysis methods were structured into three discipline groups: weight and balance, performance, and aerodynamics and stability. RBDO was implemented to manage the errors introduced by the empirical methods that were implemented in each contributing discipline. The probability distributions of these errors were estimated by using the developed analysis methods to predict published aircraft specification data. The discrepancies between the predicted characteristics and the published characteristics were used to estimate the error probability functions for each error source considered. Four sources of error were considered, covering all of the implemented empirical analysis methods including the aircraft empty weight estimation, rubber engine sizing for thrust and fuel consumption characteristics, and the drag modeling.

The geometry of the wings, fuselage, and empennage, the sea level thrust, and the fuel capacity were defined by a set of 14 design variables. In addition, the coupling of the discipline analysis methods introduced 10 coupling variables. The design and coupling variables are shown in Table 5.2. Section 5.2 describes the algorithm that builds a complete aircraft model given the specified goals and a design variable vector. 


\subsection{Automated Aircraft Configuration}

The design and coupling variables are not sufficient to fully describe an aircraft model. Many unknowns remain that are required for the discipline analysis methods to function. An algorithm was developed that determines the unknown terms using a set of configuration rules and geometry. The rules include empirical equations for the sizing and positioning of the landing gear, fuel tanks, and other subsystems. Additional rules were derived from airworthiness requirements and were used to size the fuselage and position the aisles, seats, and exits while maintaining or exceeding airworthiness standards for headroom, aisle width, and exit proximity. This ensures that when the optimizer adjusts the fuselage shape, a feasible fuselage layout is established. The regulations relevant for the sizing of the fuselage are provided in Table B.1 in the appendix. This enables the automatic development of $3 \mathrm{~d}$ conceptual models that describe the overall geometry of the aircraft with sufficient detail for the discipline analysis packages to run. An example of the algorithm output is shown in Figure 5.2.1, including the cross section of the fuselage.
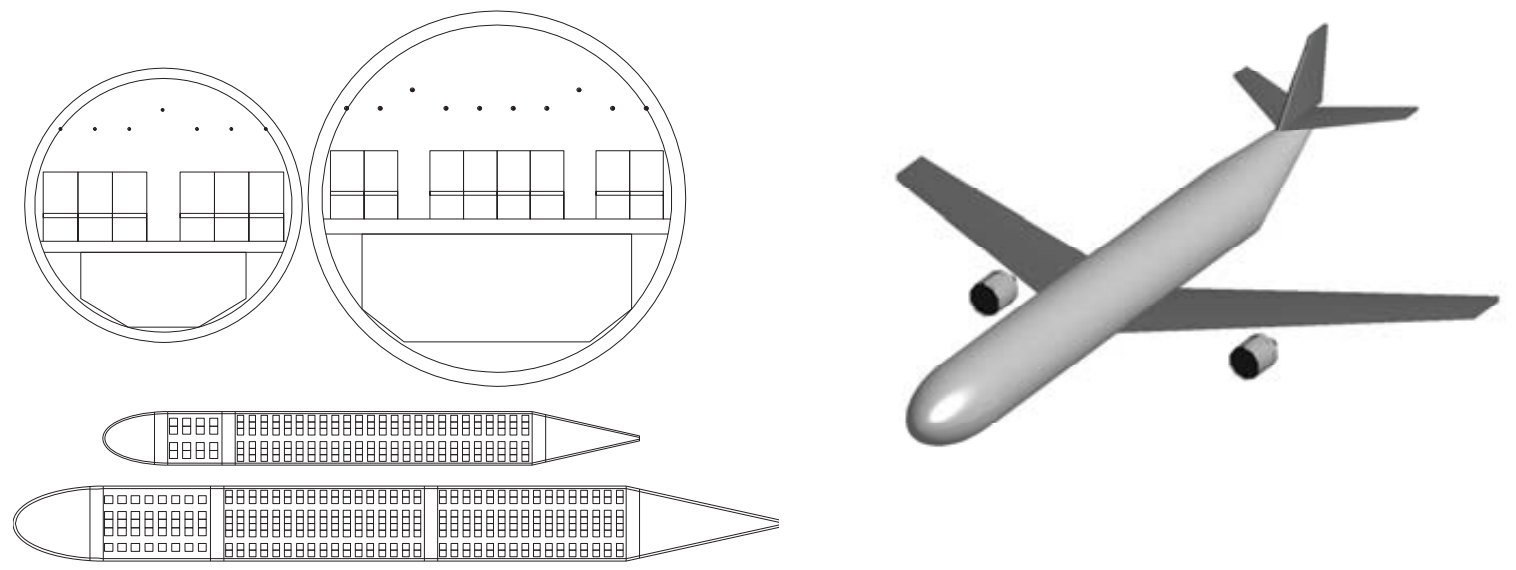

Figure 5.2.1: Aircraft Layout Example 


\subsection{Contributing Analysis Methods}

The analysis methods were grouped into 3 disciplines: Aerodynamics and Stability, Weight and Balance, and Performance. The methods implemented in each discipline, the local constraints, and the coupling variables are described in the following sections.

\subsubsection{Aerodynamics and Stability}

The aerodynamics discipline computes the lift and drag characteristics of the aircraft. It consists of two main components: a non-planar vortex lattice aerodynamics solver with a compressibility correction and a component build-up method for determining the parasite drag based on methods from [11] and [137]. The vortex lattice method is a classical computational method for computing flow properties and aerodynamic forces, and is described in [138]. The Athena Vortex Lattice program, a well-known vortex lattice solver, was used [139]. The method is much faster in both model development and solution time than Computational Fluid Dynamics (CFD) and provides a sufficient degree of accuracy and flexibility for the conceptual design phase. The aerodynamics discipline solves for induced drag, parasite drag, lift and drag coefficients, aerodynamic center, and stability derivatives as a function of aircraft geometry, the responses from the performance and weight disciplines, and the specified performance targets. Static and dynamic stability constraints are enforced. The effectiveness of the control surfaces at cruise and climbout conditions are evaluated to ensure adequate pitch authority in slow flight and yaw authority in single-engine flight. The Aerodynamics and Stability discipline is dependent on the system variables, the output of the other disciplines and the design requirements. The coupling requirements of the aerodynamics discipline, the local constraints, and the output coupling variables are shown in Figure 5.3.1. The aerodynamics local constraints are shown in Table 5.3. 


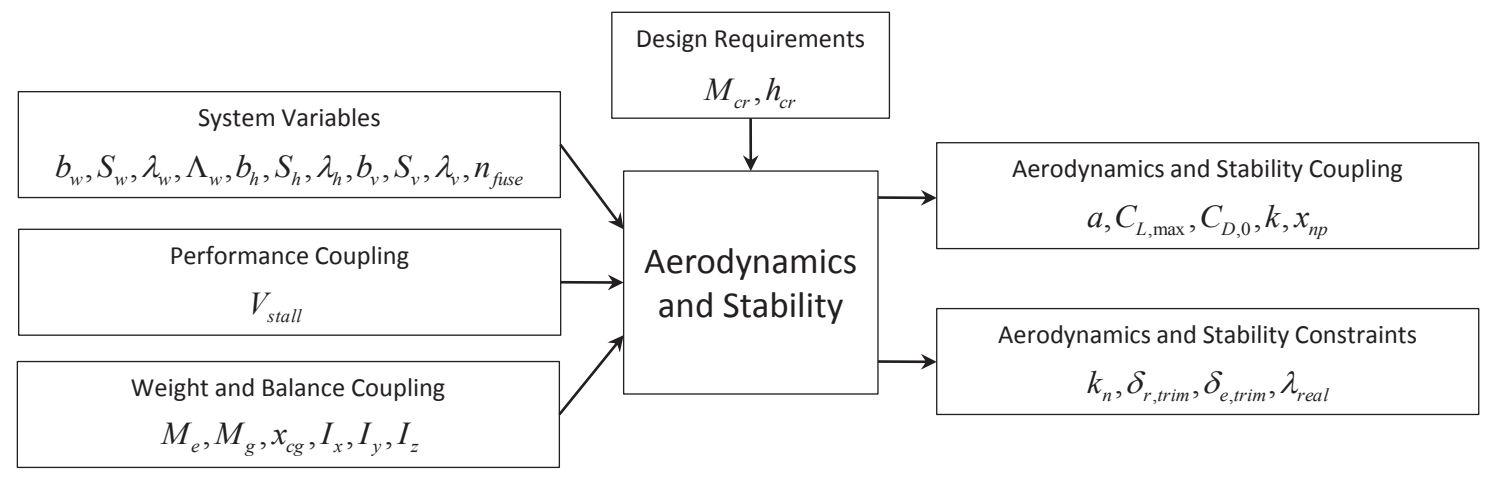

Figure 5.3.1: Aerodynamics and Stability Coupling

Both static and dynamic stability constraints are enforced. Longitudinal stability is constrained to a static margin of $5 \%$. Yaw static stability is enforced at a full thrust climb scenario with a failed engine. The constraint ensures that the rudder and vertical tail have adequate authority. Dynamic stability analysis is carried out by solving for the roots of the solution of the linearized equations of motion. The dynamic stability eigenvalues were obtained from the vortex lattice aerodynamics solver. To avoid introducing a very large number of aerodynamics constraints and the corresponding increase in computational cost associated with RBDO procedures, dynamic stability was handled in a single constraint. The constraint was formulated by obtaining the largest real part of the obtained eigenvalues and constraining it to be less than zero, ensuring decaying oscillatory or damped motion for every mode.

\subsubsection{Sources of Uncertainty}

The uncertainty due to the approximate methods used in the aerodynamics and stability discipline are somewhat difficult to quantify. The availability of aerodynamics data such as lift-to-drag ratios, induced drag constants, and parasite drag constants for cruise conditions is very limited and virtually non-existent off-cruise configurations. However, since 
the majority of a typical commercial aircraft route is spent in a cruise configuration, the consideration of uncertainty was limited to the cruise phase. The lift and drag characteristics of commercial aircraft are not widely published. However, typical cruise thrust settings are available for some aircraft - reported at specified altitudes and Mach numbers. These quantities were obtained for several Boeing airliners from the airport planning manuals available from the manufacturer's internet address [140]. With the required cruise thrust, $T_{r e q, d b}$, known for several aircraft at specific altitudes and speeds, predictions were made by modeling the aircraft and running the aerodynamics discipline to calculate the predicted required thrust, denoted by $T_{r e q, p}$, for the given cruise speed and altitude. The number of data available for comparison was limited to 7 samples - too few to develop an accurate PDF function. Therefore, the error term was assumed to be uniformly distributed between the highest and lowest observed error. The error term was defined as the ratio of predicted required thrust to the observed thrust from the database. The required thrust output from the aerodynamics discipline was then scaled by this ratio to correct for the error as shown in equation 5.3.1. The error term was considered to be a uniformly distributed random parameter.

$$
\begin{gathered}
\varepsilon_{T_{r e q}}=T_{r e q, p} / T_{r e q, d b} \\
T_{r e q}=T_{r e q, p} / \varepsilon_{T_{r e q}}
\end{gathered}
$$

Considering the error in the predicted thrust requirements in the cruise configuration enables the management of the errors in both the predicted parasite drag and induced drag models implemented in the aerodynamics discipline. The error contributions in non-cruise conditions was neglected. 


\subsubsection{Weight and Balance}

The aircraft structural weight is estimated by using a statistical group weights method. Both the weight and center of gravity of each major aircraft component are calculated by statistical relationships to establish an estimate of the overall empty weight of the aircraft. These methods are described in many aircraft conceptual design publications $[11,12,141]$. The dependencies of the weight and balance discipline is shown in Figure 5.3.2. In general, the statistical equations are functions of the geometry and performance requirements of the aircraft, including payload capacity, cruise speed, and altitude. The empty and gross mass, the center of gravity, and the moments of inertia of the aircraft are calculated.

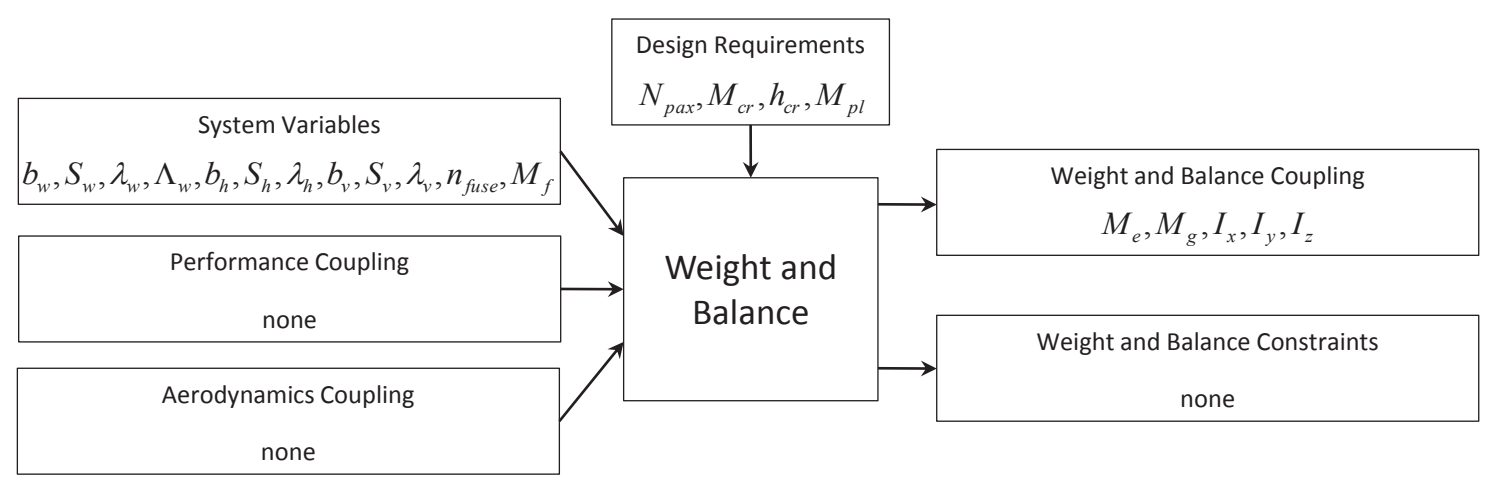

Figure 5.3.2: Weight and Balance Coupling

\subsubsection{Sources of Uncertainty}

The designs from the compiled aircraft database were modeled and the predicted empty mass of each design was compared with the observed value from the database. The histogram of the errors indicates that the empty mass error term can be approximated by a normal distribution curve with a mean of 1 and a standard deviation of 0.0636 . The mass error term was defined as shown in equation 5.3.2. Empty mass calculations carried out in 
the optimization were scaled by the error term to account for the uncertainty in the prediction of the aircraft empty mass.

$$
\begin{gathered}
\varepsilon_{M_{e}}=M_{e, p} / M_{e, d b} \\
M_{e}=M_{e, p} / \varepsilon_{M_{e}}
\end{gathered}
$$

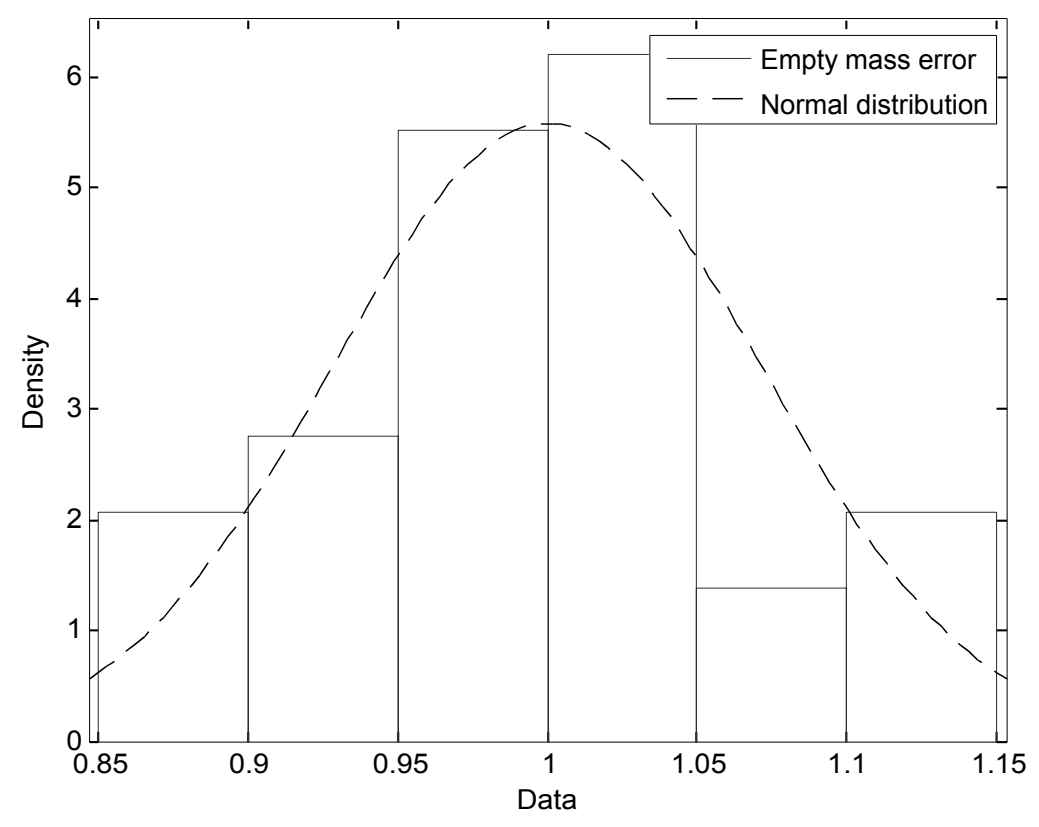

Figure 5.3.3: Mass Error Distribution Estimates

\subsubsection{Performance}

Performance estimation is carried out by a configurable set of two dimensional flight simulation components that include takeoff performance, climb, cruise, descent, and landing under normal flight conditions over the intended route of the new aircraft design. The algorithm calculates the net force acting on the aircraft by finding the drag forces, lift forces, 
and available thrust forces over a numerical simulation. Simulation of contingency flight situations is also carried out to determine reserve fuel requirements for a $200 \mathrm{~km}$ diversion scenario. Climb performance requirements under engine failure circumstances were also considered. The flight simulation route profiles under normal conditions and contingency conditions are shown in Figure 5.3.4. A turbofan performance module was developed. It implements a rubber engine sizing technique that uses statistical equations to estimate the mass, cruise thrust, and cruise fuel consumption of hypothetical engines meeting the thrust requirements of the conceptual design. Off-cruise thrust and fuel consumption is estimated by interpolating and scaling engine thrust tables for several engines to predict the available thrust at any altitude and Mach number.

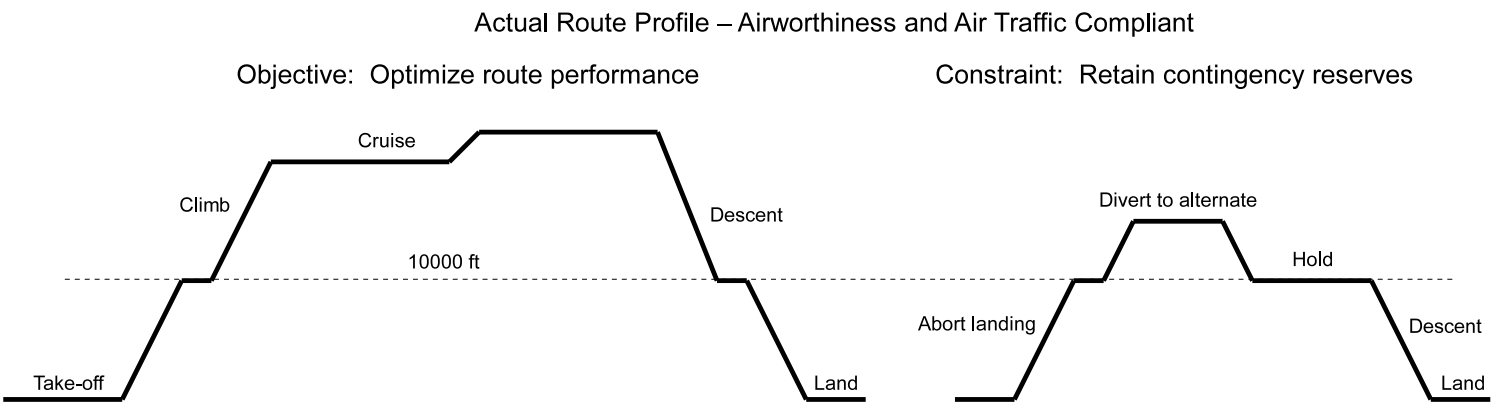

Figure 5.3.4: Flight Profile

The coupling dependencies of the performance discipline are shown in Figure 5.3.5. The performance constraints were set to maintain or exceed the performance characteristics of the Boeing 737-800. In addition, the single engine climb performance was constrained to comply with airworthiness standards. The Mach number perpendicular to the flight surfaces was constrained to remain below 0.70 to maintain flow conditions within the range of validity for the vortex lattice solver and the compressibility correction equation. Higher cruise Mach numbers are possible by increasing the sweep angle of the wings to maintain a perpendicular Mach number below the limit. A summary of the local performance 
constraints, designed to enforce the performance characteristics of the Boeing 737-800, is given in Table 5.4 .

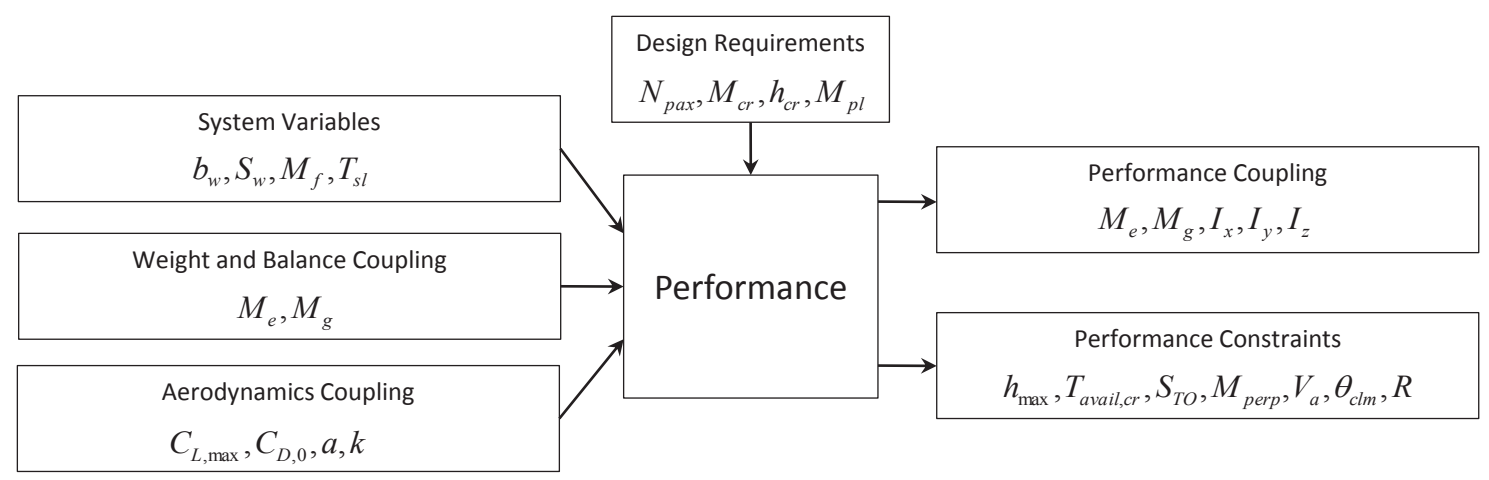

Figure 5.3.5: Performance Coupling

\subsubsection{Sources of Uncertainty}

There are several sources of uncertainty present in the performance analysis methods. The simulation is dependent on the size of the time step and the relative error tolerances. However, this source of error was assumed to be small relative to the errors introduced by the statistical equations utilized to estimate the engine performance characteristics. Uncertainties in the aerodynamics and mass properties certainly influence the performance discipline as well, but these are accounted for in their respective disciplines. Inter-discipline coupling ensures that the influence of errors handled in one discipline spreads to the others. The sources of error considered in the performance discipline were therefore related to statistics based rubber engine sizing methodology.

The engine selection is driven by the $T_{s l}$ design variable. All of the other performance characteristics of the engine are calculated using the statistical relationships for the cruise performance characteristics, and by interpolating engine performance charts for performance in off-design conditions. The error considered was restricted to cruise conditions, 
where ample data is available to accurately predict the error PDFs. Statistical relationships for predicting engine performance are widely available in conceptual design literature $[11,12,142]$. The implemented approach is based on the equations proposed by Svobota supplemented by additional engine data acquired from more recent design specifications. This data is given in Table A.2 in the appendix. As before, the performance predicted by the algorithm was compared with a database of published specifications. Error ratios were defined to scale the predicted available cruise thrust, $T_{\text {avail }, p}$, and predicted cruise fuel consumption, $S F C_{p}$, to account for the uncertainties introduced by the statistical predictions.

$$
\begin{gathered}
\varepsilon_{T_{\text {avail }}}=T_{\text {avail }, p} / T_{\text {avail }, d b} \\
T_{\text {avail }}=T_{\text {avail }, p} \cdot \varepsilon_{T_{\text {avail }}} \\
\varepsilon_{S F C}=S F C_{p} / S F C_{d b} \\
S F C=S F C_{p} / \varepsilon_{S F C}
\end{gathered}
$$

The frequencies of the error ratio between the predicted and observed values were plotted in a histogram. Figure 5.3.6 indicates that the error ratios arising from thrust and fuel consumption predictions can be represented by normal distribution functions with mean values of 1 and standard deviations of 0.0836 and 0.0780 respectively. The error associated with off-cruise thrust and fuel prediction was not considered. However, since the majority of the aircraft route with respect to time, distance, and fuel burn, is spent in cruise configuration, it can be expected that the errors associated with the off-design engine performance predictions would be small by comparison. 


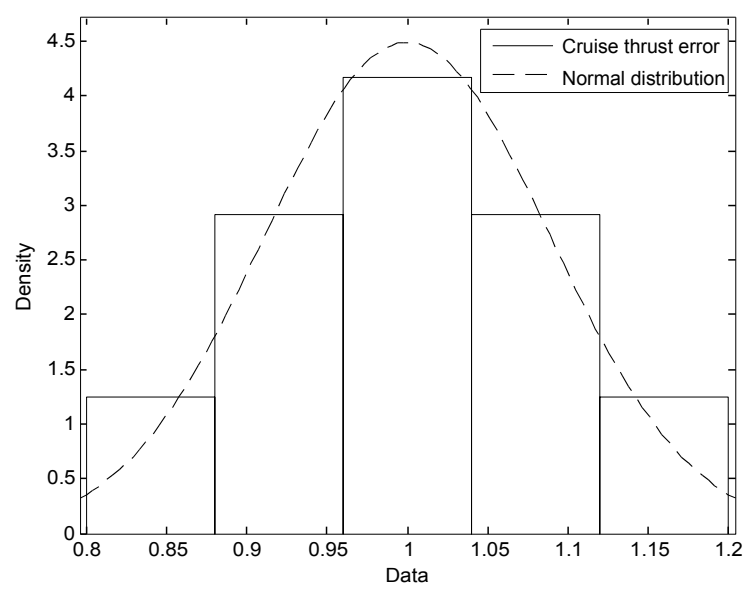

(a) Available Cruise Thrust Error

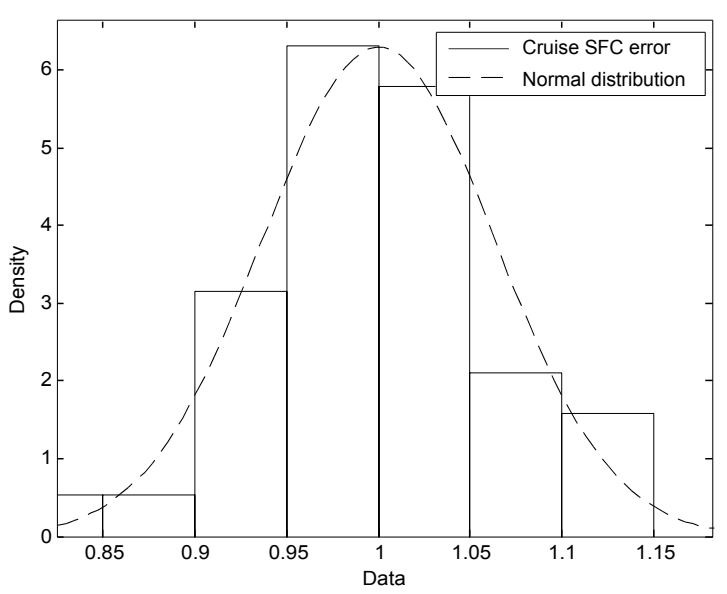

(b) Cruise SFC Error

Figure 5.3.6: Propulsion Error Distribution Estimates

\subsection{Solution Strategy}

The aircraft conceptual design optimization was carried out using two completely distinct methods for comparison. The first implements the RyeMDO IDF/Sequential/PMA modules with a SQP optimizer. The second method implements a multi-objective GA with the MCS reliability assessment module using approximation models. Multi-level methods such as CO or BLISS were not considered for the aircraft conceptual design case study. The optimization is less suitable for multi-level methods because there are very few local design variables and many coupling variables. Solutions were obtained with a 4-core Intel i7 desktop computer. Solutions were obtained over a range of reliability indices to show the progression from a deterministic solution to solutions with reducing error tolerances. The methods are described in Sections 5.4.1 and 5.4.2. 


\subsubsection{Method 1: IDF/Sequential/PMA}

The first method implemented RyeMDO to solve the aircraft conceptual design problem using the IDF/Sequential/PMA blocks as described in Chapter 3.1 with some enhancements to accommodate the specific properties of the problem. The algorithm was shown to be reliable and less sensitive to coarse objective and constraint functions than the double loop approaches. Additionally, it was found to be less sensitive to the selection of a starting vector than the single loop approach, and more efficient than all but the single loop approach on the test problems studied. The sequential method has some additional advantages when solving for multiple reliability levels. Since the sequential method solves a sequence of full deterministic optimizations followed by reliability assessments until convergence, the solutions progressively pass through lower reliability indices until they reach the target level. By enforcing first a low reliability index followed by progressively larger reliability indices, it is possible to extract intermediate solutions to plot the progressive relationship between the optimum design and the selected target reliability index without greatly increasing the number of function evaluations. In this manner, a multi-objective optimization was solved. The objective functions were defined as minimizing the fuel consumption for the specified flight profile while maximizing the reliability index of the designs.

The MDF method was implemented for the reliability assessment sub-problems rather than IDF. For problems having large numbers of coupling variables and small numbers of uncertain variables or parameters, the dimensionality of the reliability sub-problem is greatly increased when the IDF method is used. For example, the aircraft conceptual design problem formulation has 10 coupling variables and 4 uncertain parameters. Therefore, the PMA reliability assessment must solve an optimization with 14 dimensions for every constraint. Additionally, the auxiliary equality constraints required to enforce coupling variable consistency have to be considered. This creates an optimization problem of significant com- 
plexity that must be solved for every constraint for each iteration of the sequential method. Implementing the MDF method for the reliability assessments reduces the dimensionality of the PMA optimization problem to just the 4 uncertain parameters and does not include any auxiliary constraints. It was found that the PMA reliability assessments converged faster and with greater reliability using this approach. The IDF method was retained as the system optimization strategy. Recall that for sequential RBDO, the system problem must solve each constraint independently based on different shifted uncertain parameters. In an MDF based scheme, the system problem requires separate MDA runs for the objective and each constraint, introducing great computational expense, leading to the selection of an IDF-based scheme for the main optimization loop. The procedure is outlined as follows.

1. Determine analysis error distributions

(a) Model each entry in the aircraft specification database

(b) Predict performance

(c) Calculate ratio of predicted performance vs. published specifications for each uncertain analysis method considered

(d) Generate histogram and apply a best fit PDF curve for each source of uncertainty considered

2. Select starting vector and a list of reliability indices to be enforced.

3. Run IDF based deterministic optimization.

4. Run MDF based PMA reliability assessment at the current reliability index, shift variables according to the sequential approach.

5. Check for convergence with current reliability goal. 
(a) yes, update starting vector with the current optimum point, select next reliability goal, return to 3 .

(b) no, return to 3 .

6. Advance to next target reliability level

(a) retain solution as new starting vector

(b) return to 3

The block diagram is shown in Figure 5.4.1. The method was run deterministically and for probabilities of $0.7,0.8,0.9,0.95$, and 0.99 and compared with a second, independent approach, outlined in Section 5.4.2.

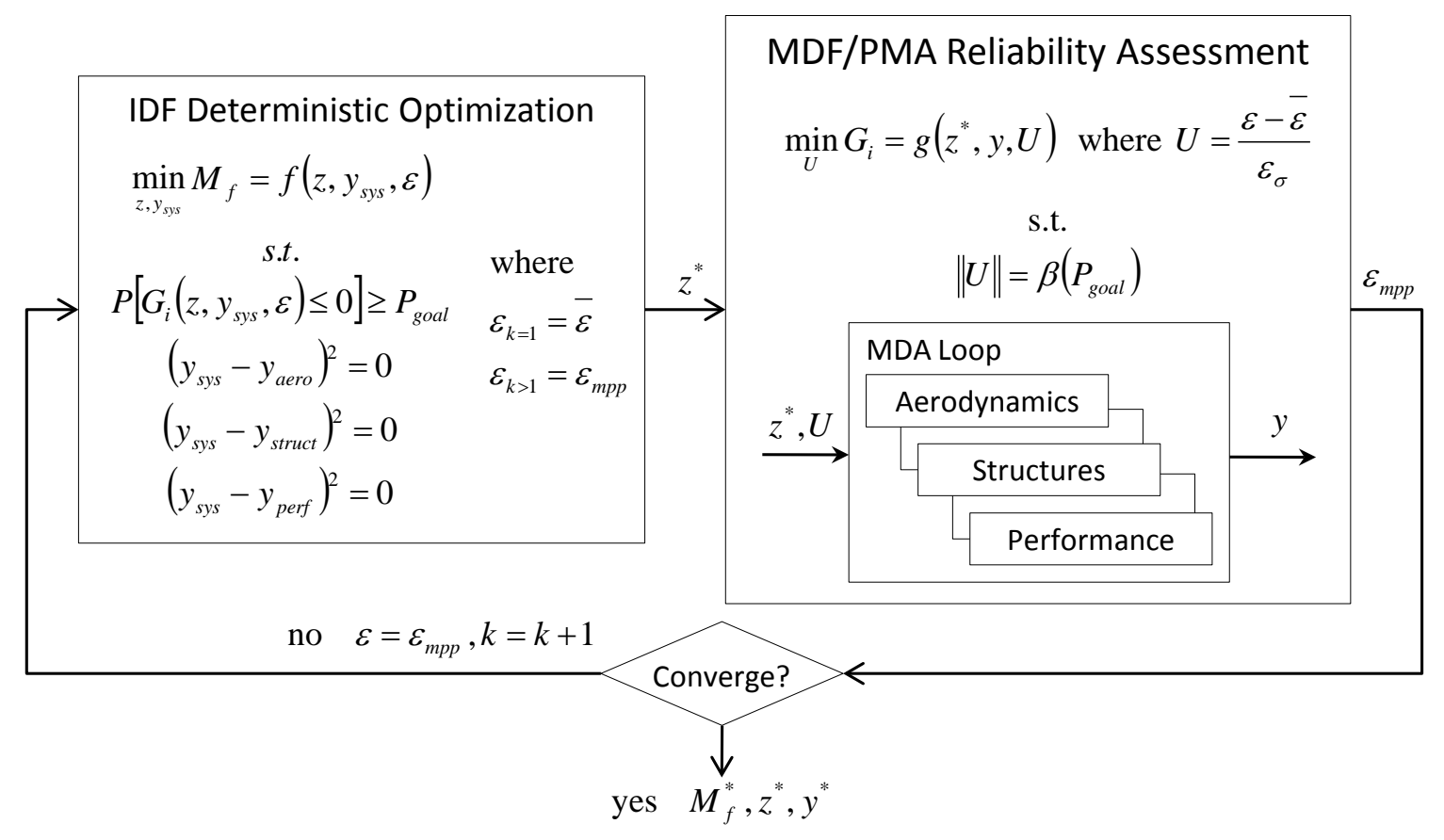

Figure 5.4.1: Block Diagram - Method 1 


\subsubsection{Method 2: MCS/GA}

The second solution strategy implemented a multi-objective GA with the RyeMDO MCS block using approximation models. The approach introduces the reliability index, $\beta$, as a second objective function. The problem formulation is shown in equation 5.4.1 where $M_{f}$ is the fuel mass and $\beta$ is the reliability index.

$$
\begin{aligned}
& \min \left[M_{f},-\beta\right]=f(\bar{x}, \bar{P}) \\
& \text { s.t.P }\left(g_{i}(x, P) \leq 0\right) \geq \beta
\end{aligned}
$$

The MDF method was used rather than IDF or CO, which are far less suitable for solving the MCS/GA based approach for the following reasons. Firstly, GAs are, in general, not very efficient for solving problems having equality constraints [143]. Both the IDF and $\mathrm{CO}$ strategies require introducing auxiliary equality constraints to enforce inter-discipline compatibility. Secondly, the MCS based approach involves building accurate RSA models with respect to the uncertain variables and parameters at every design point. In an IDF approach, the dimensionality of the RSA models would have to include the coupling variables, and would therefore be compromised in accuracy and require large sample sizes to calculate. By only considering feasible points using MDA loops, the dimensionality of the RSA models under an MDF optimizer is reduced to 4 - the number of uncertain parameters considered in the aircraft conceptual design case study. The accuracy of the surrogate models is maintained by recalculating a local DOE surrounding every new design point to generate new models as the Pareto front shifts. However some minimal additional uncertainty is introduced by carrying out the reliability assessment on surrogate models [144]. This is mitigated by carrying out a reliability assessment on the system equations for each solution on the output Pareto front. If any discrepancy is discovered, the location of each 
solution is shifted to the corrected reliability index. The outline of the approach is described as follows.

1. Determine error PDF functions as with Method 1

2. Obtain a design variable vector $\bar{z}$ from the GA population (mean value).

3. Run a MDA to obtain a feasible coupling variable vector $y$.

4. Evaluate the design to determine the fuel consumption objective, $M_{f}$, at the current location.

5. Evaluate the constraints at the current location.

(a) If any constraint fails, the design is infeasible. Assign a penalty to $\beta$ and return to 1 .

(b) If all constraints pass, proceed to 5 .

6. Build a DOE around the current design point.

(a) Calculate a grid of evenly spaced uncertain variable and parameter vectors in normal space, keeping deterministic quantities fixed.

(b) Check if the constraints at any of the grid points have already been evaluated.

(c) Evaluate each constraint at every grid point in the DOE not previously calculated.

7. Calculate RSA approximation models for every constraint.

8. Sample every constraint RSA model with $N$ uncertain variable and parameter vectors randomly generated w.r.t corresponding PDF functions where $N$ is a very large number. 
9. Count the number of passes, $N_{\text {pass }}$, and failures $N_{\text {fail }}$.

(a) If any constraint fails, count as a design failure.

(b) If all constraints pass, count as a design pass.

10. Calculate $P_{\text {fail }}=\frac{N_{\text {fail }}}{N}$

11. Calculate $\beta$

12. Return to 1 until specified number of generations have elapsed.

13. Run a deterministic reliability analysis to verify the estimated reliability indices for each solution on the Pareto front

The procedure does not yield a single point solution at a given reliability index. Rather, it produces a Pareto front. A Pareto front refers to a range of designs, each having a different trade-off between the objective function performance (fuel mass) and the reliability index. The MCS based approach has several additional advantages with respect to the FORM based reliability assessment approaches. When using FORM, the computational effort required by the MDF method can be prohibitive. Since each constraint is evaluated with a different set of uncertain variables and parameters, MDAs must be performed once to evaluate the objective function, and once to evaluate every constraint individually every time the design variables are changed. Each MDA was found to require 5 or 6 calls to each discipline on average for the aircraft conceptual design problem. Consequentially, optimization problems having many uncertain constraint functions suffer in performance. This is not the case for MCS based methods, where only one MDA loop needs to run to find feasible coupling variables. The objective and all constraint functions are all evaluated at this point. However, the MCS method requires a very large number of constraint function evaluations to accurately estimate the probability of failure. This is mitigated by carrying 
out the MCS on surrogate models rather than the constraint functions themselves. Since the aircraft conceptual design problem has 4 uncertain parameters, sample sizes of 25 or so were found to be sufficient to obtain accurate RSA surrogate models. The surrogate models were randomly sampled with a large sample of uncertain parameter vectors $\left(10^{6}\right.$ was used). A block diagram describing the method is shown in Figure 5.4.2. Section 5.5 outlines the results and compares the solutions from both approaches.

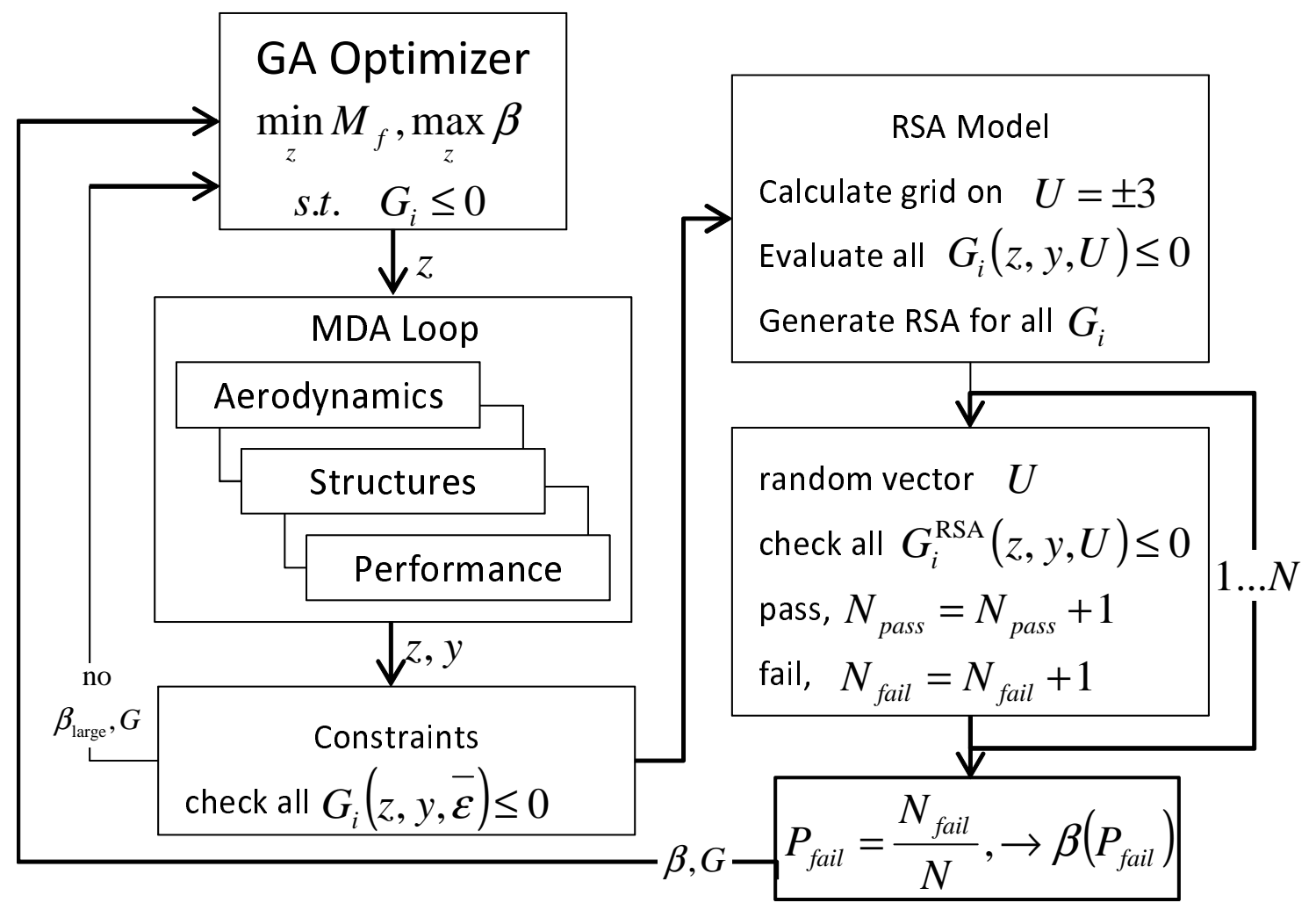

Figure 5.4.2: Block Diagram - Method 2

\subsection{Results}

The IDF/Sequential method was run for probabilities of $0.7,0.8,0.9,0.95$, and 0.99 corresponding to reliability indices of $0.52,0.84,1.28,1.64$, and 2.32 respectively, minimizing 
the fuel capacity required for a $5670 \mathrm{~km}$ route with a reserve fuel capacity sufficient for a $200 \mathrm{~km}$ diversion. The standard deviations of the considered error uncertain parameters rendered it impractical to consider larger reliability indices, since convergence was no longer possible. These results were compared with the Pareto-front obtained by the GA/MCS procedure. Figure 5.5.1 compares the two results to the fuel capacity of the Boeing 737-800, also obtained for a maximum payload cruise of $5670 \mathrm{~km}$ and a $200 \mathrm{~km}$ contingency reserve. Both results exhibited reasonable agreement between the FORM based approach and the MCS based approach. An additional source of discrepancy between the two methods may be the use of a GA optimizer for the MCS based approach. GAs are global optimization techniques and are therefore less prone to premature convergence finding a local rather than global optimum point.

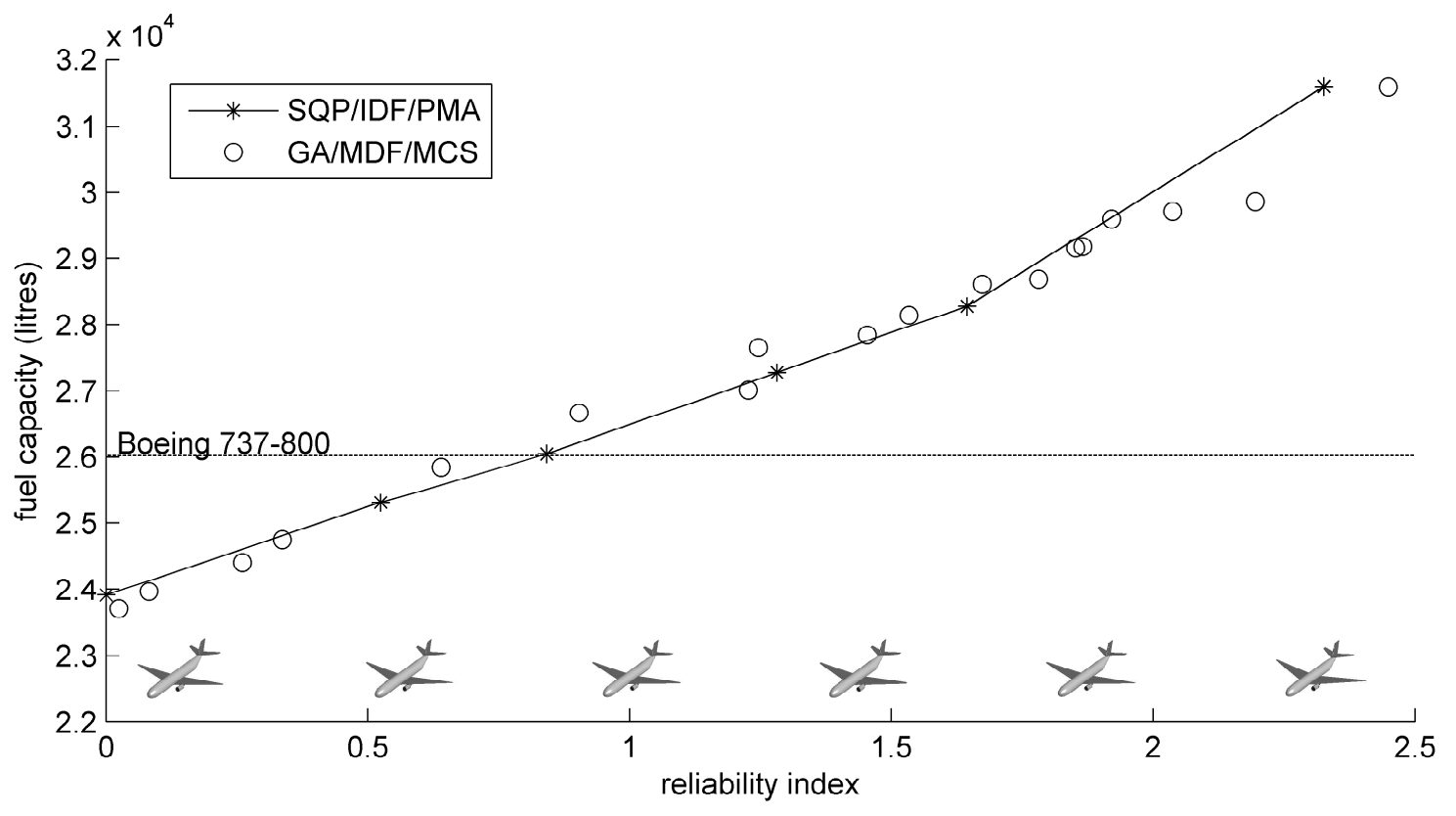

Figure 5.5.1: Fuel vs. Reliability Index

The deterministic solution predicts that an optimized aircraft can improve on the fuel consumption of the Boeing by 7.7\%. However, the uncertainties in the analysis methods used to obtain the result introduced uncertainty that renders the prediction of the deterministic 
design unreliable, since the design lies very close or directly on several of the probabilistic constraint boundaries, as shown in Figure 5.5.2, where the straight dashed lines represent the design goals for field length, approach speed, and range. Consequentially, errors present in the analysis methods are likely to cause the deterministic design to violate one or more of the constraint boundaries if the design was subjected to more detailed analysis methods. However, increasing the reliability level pushes each constraint farther into feasible design space, resulting in a conceptual design requiring more fuel than the deterministic design, while increasing the likelihood that the design will be feasible when subjected to better analysis. At a probability of $80 \%$ ( $\beta=0.84)$, the predicted optimum design has a fuel capacity roughly equivalent to the Boeing 737-800.
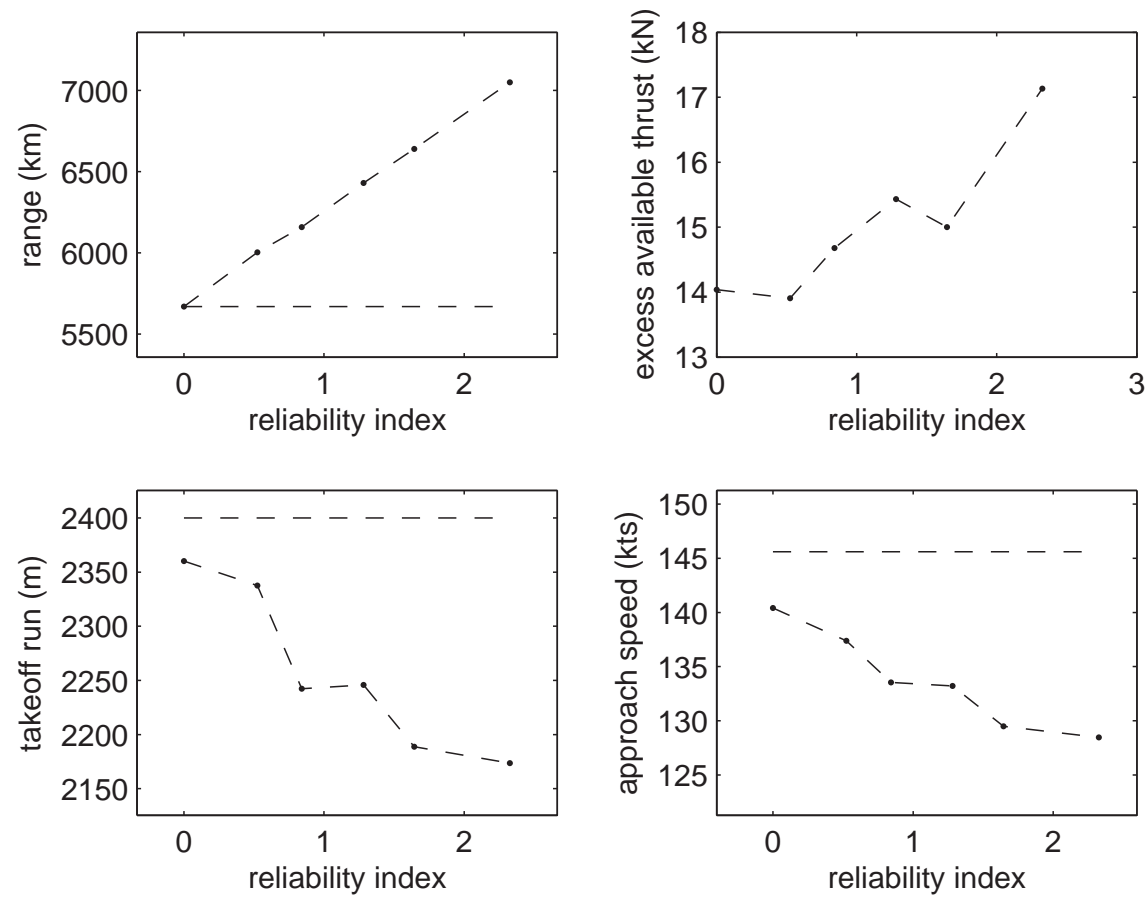

Figure 5.5.2: Uncertain Constraints vs. Reliability Index

The results can be interpreted as follows. Given the uncertainties in the analysis approaches, to ensure that the range of the aircraft can reach the design goal of $5670 \mathrm{~km}$, 
the conceptual design range target must be increased to $6000 \mathrm{~km}$ for $\mathrm{P}=0.70$, to $6200 \mathrm{~km}$ for $\mathrm{P}=0.80$, and all the way to $7000 \mathrm{~km}$ for $\mathrm{P}=0.99$, thereby producing a more conservative design. As the reliability level continues to increase to $99 \%$, the changes to the design necessary to accommodate the changing constraint boundaries, become more pronounced. Figure 5.5.3 shows the influence of increasing the reliability level on the geometry, mass, sea level thrust, and drag properties of the aircraft. The wing area, empty mass, gross mass, fuel mass, and sea level required thrust all increase. This yields a more conservative design than the deterministic solution but improves the chances that the design will be found to be viable when subjected to better analysis. Note that the probability values given only indicate the likelihood that that the uncertainties considered will not cause failure, assuming the obtained probability distributions and the FORM approximations are accurate. Figure 5.5.4 compares the geometry of the deterministic design and several reliable designs to the Boeing 737-800. The Figure indicates that considering uncertainties in conceptual design significantly impacts the size and shape of the optimum aircraft wing design. Increases in both span and area impact the probabilistic constraints governing takeoff, required thrust at cruise, ceiling, and approach speed.

\subsection{Summary}

An aircraft conceptual design optimization problem was developed to demonstrate how RBDO can be used to manage errors that arise from using traditional low fidelity conceptual analysis methods. The uncertainties considered included analysis methods that depend on statistical or simplified analytical equations to solve, including drag, weight, engine cruise thrust, and engine cruise fuel consumption. Error terms were defined as the ratio of predicted performance to observed performance, taken from aircraft and engine databases. 

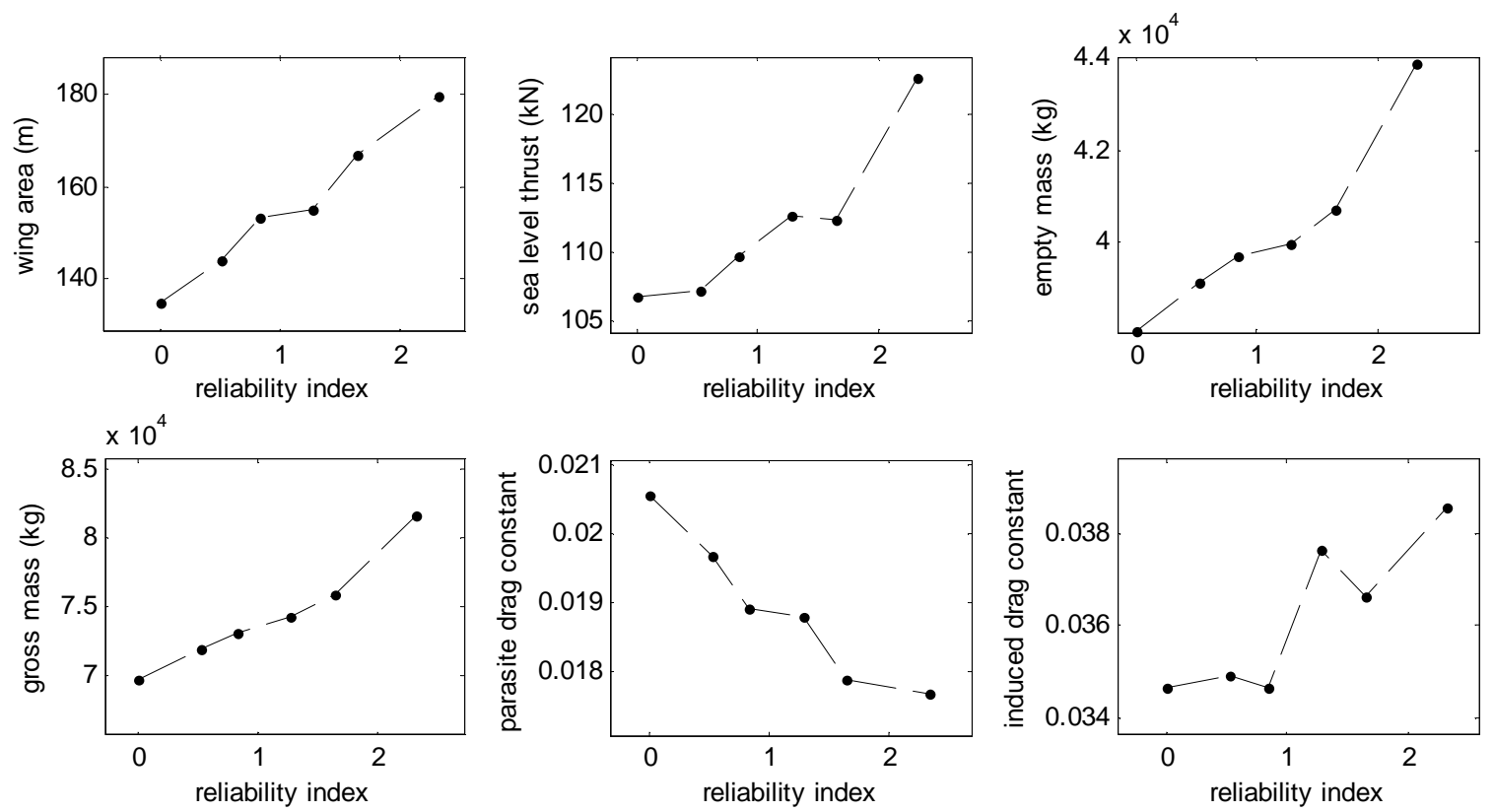

Figure 5.5.3: Aircraft Specifications vs. Reliability Index

A commercial aircraft conceptual design problem was developed and solved using RyeMDO. The performance targets and the passenger capacity was defined to match the Boeing 737800 for comparison purposes. The deterministic result indicated that an improvement over the 737 was possible, but the design was found to lie on or near several constraint boundaries, making the design vulnerable to the uncertain methods used to develop it, where any deviation from the mean error values would likely result in design failure when subjected to better analysis later in the design process. By implementing RBDO, it was shown that enforcing target reliability indices can push the optimum design deeper into the feasible region of the design space, resulting in designs that are more likely to meet the performance goals when subjected to better analysis.

It should be noted that, as with the wing box example outlined in Chapter 4, optimum designs were much more frequently observed to be optimistic - over-predicting the performance of the design. Since the PDF functions were normal distributions, it might be expected that the predictions would be equally likely to under-estimate or over-estimate 


\section{Deterministic $\quad \mathrm{P}=0.80$}
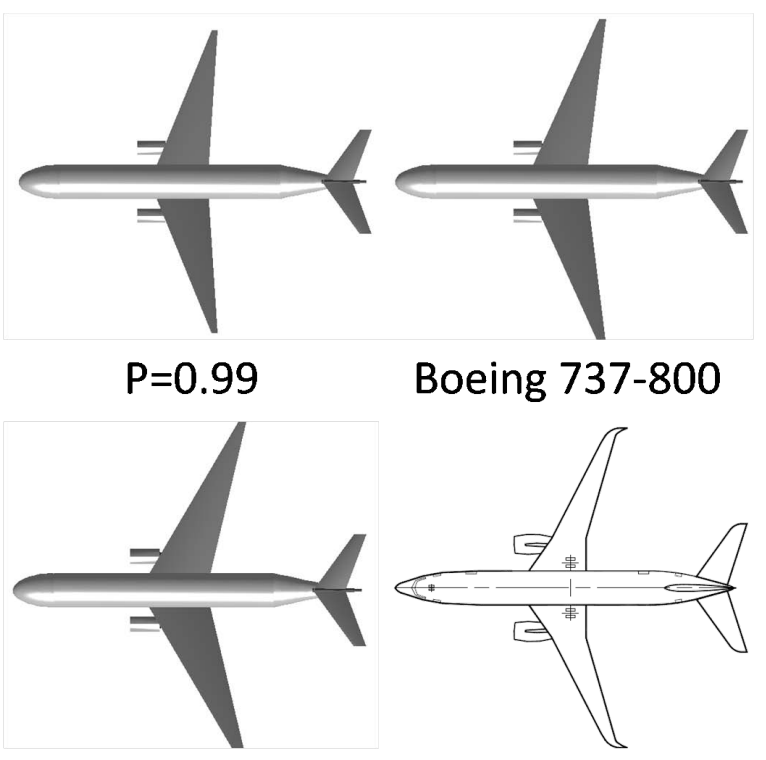

Figure 5.5.4: Results Compared With the Boeing 737-800

performance. This was almost never the case in the problems studied in this research. This is due to the fact that the optimization methods used search for the best possible objective function performance given the provided analysis methods. The optimizer will therefore move into design space that has advantage to the objective function whether the design is truly better than the adjacent designs or the design falls within a region where the analysis methods over-predict the performance of the design. The optimizer sees no distinction. This renders it more likely for a solution to lie in a region where performance characteristics are over-predicted. This would consistently yield designs that are optimistic, and promise performance characteristics that are not likely to be achieved when the design is subjected to better analysis methods. Consequentially, it is important to implement procedures such as RBDO to manage uncertain analysis methods in order to find the best possible designs that lie no closer to the constraint boundaries than the acceptable reliability index defined by the designer. 
Table 5.2: Design and Coupling Variable List

\begin{tabular}{|c|c|c|c|c|}
\hline \multirow{2}{*}{$\begin{array}{l}\text { design } \\
\text { variable }\end{array}$} & \multirow[t]{2}{*}{ description } & \multirow[t]{2}{*}{ unit } & \multicolumn{2}{|c|}{ limits } \\
\hline & & & lower & upper \\
\hline$A R_{w}$ & wing aspect ratio & - & 5 & 10 \\
\hline$S_{w}$ & wing area & $m^{2}$ & 100 & 300 \\
\hline$\lambda_{w}$ & wing taper ratio & - & .1 & .9 \\
\hline$\Gamma_{w}$ & wing dihedral & deg & 0 & 5 \\
\hline$\Lambda_{w}$ & wing sweep & deg & 0 & 45 \\
\hline$A R_{h}$ & horizontal tail aspect ratio & - & 5 & 10 \\
\hline$S_{h}$ & horizontal tail area & $m^{2}$ & 20 & 90 \\
\hline$\lambda_{h}$ & horizontal tail taper ratio & - & .1 & .9 \\
\hline$A R_{v}$ & vertical tail aspect ratio & - & 2 & 10 \\
\hline$S_{v}$ & vertical tail area & $m^{2}$ & 20 & 90 \\
\hline$\lambda_{v}$ & vertical tail taper & - & 0.1 & 0.9 \\
\hline$M_{f}$ & fuel mass & $k g$ & 10000 & 35000 \\
\hline$T_{s l}$ & engine sea level thrust & $N$ & 80000 & 160000 \\
\hline$n_{\text {fuse }}$ & fuselage configuration index $*$ & - & 1 & 7 \\
\hline \multirow{2}{*}{$\begin{array}{l}\text { coupling } \\
\text { variable }\end{array}$} & \multirow[t]{2}{*}{ description } & \multirow[t]{2}{*}{ unit } & \multicolumn{2}{|c|}{ limits } \\
\hline & & & lower & upper \\
\hline$M_{e}$ & empty mass & $\mathrm{kg}$ & 10000 & 80000 \\
\hline$M_{g}$ & gross mass & $k g$ & 20000 & 12000 \\
\hline$x_{c g}$ & center of gravity location & $m$ & 0 & 40 \\
\hline$V_{s}$ & stall speed & $m / s$ & 20 & 120 \\
\hline$C_{L, \max }$ & maximum lift coefficient & - & 1 & 3 \\
\hline$C_{D, 0}$ & drag coefficient & - & 0.001 & 0.1 \\
\hline$k$ & induced drag constant & - & 0.001 & 0.1 \\
\hline$I_{x}$ & moment of inertia about $\mathrm{x}$ & $\mathrm{kg} \cdot \mathrm{m}^{2}$ & $10^{5}$ & $10^{8}$ \\
\hline$I_{y}$ & moment of inertia about y & $\mathrm{kg} \cdot \mathrm{m}^{2}$ & $10^{5}$ & $10^{8}$ \\
\hline$I_{z}$ & moment of inertia about $\mathrm{z}$ & $\mathrm{kg} \cdot \mathrm{m}^{2}$ & $10^{5}$ & $10^{8}$ \\
\hline
\end{tabular}

Table 5.3: Aerodynamics and Stability Local Constraints

\begin{tabular}{lll}
\hline constraint & description & value \\
\hline$k_{n}$ & static margin & $\geq 0.05$ \\
$\delta_{r, \text { trim }}$ & rudder trim angle & $\leq \delta_{r, \text { max }}$ \\
$\delta_{e, \text { trim }}$ & elevator trim angle, 1 engine inoperative & $\leq \delta_{e, \text { max }}$ \\
$\lambda_{\text {real }}$ & motion equation eigenvalues & $<0$ \\
\hline
\end{tabular}


Table 5.4: Performance Local Constraints

\begin{tabular}{lll}
\hline constraint & description & value \\
\hline$h_{\text {max }}$ & service ceiling & $\geq 12200 \mathrm{~m}$ \\
$T_{\text {avail }, \text { cr }}$ & available thrust at cruise & $\geq T_{\text {req,cr }}$ \\
$S_{T O}$ & takeoff distance & $\leq 2400 \mathrm{~m}$ \\
$M_{\text {perp }}$ & Mach number perpendicular to flight surfaces & $\leq 0.70$ \\
$V_{a}$ & approach speed & $\leq 145 \mathrm{kts}$ \\
$\theta_{\text {clm }}$ & single engine climb gradient & $\geq 2.5 \%$ \\
$R$ & aircraft maximum range with reserves & $\geq 5670 \mathrm{~km}$ \\
\hline
\end{tabular}




\section{Chapter 6}

\section{Conclusion}

The motivation of this research was to develop a framework for aircraft conceptual design that accounts for the uncertainties introduced by low fidelity conceptual analysis methods by utilizing historical aircraft data. A multi-objective, multi-discipline reliability-based optimization tool called RyeMDO was developed. Several multi-disciplinary RBDO strategies were implemented with different combinations of MDO and reliability assessment approaches. The performance and reliability of each method were assessed by solving well known analytical optimization problems and truss optimization problems. The efficiency and the reliability of each approach was assessed by solving the optimization examples repeatedly at different starting vectors. It was found that for the problems considered, an IDF method with a sequential RBDO strategy exhibited the best combination of efficiency and reliability. The single-loop/MDF method was found to be efficient, but exhibited a loss in accuracy relative to the exact FORM based methods. Additionally, it was found that the single loop approach was somewhat sensitive to the location of the starting vector, and the solutions exhibited some scatter. The CO based approaches frequently failed to converge.

A method for assessing the uncertainties introduced by low fidelity analysis methods was 
introduced. The method uses historical data to estimate the statistical distributions of error between known data and the predictions of uncertain analysis methods. Two case studies were considered: the conceptual design optimization of the wing box of a light jet.

The first case study considered a multi-objective wing box optimization consisting of an aerodynamics discipline using a vortex-lattice solver and a FEM based structural analysis solver. The FEM analysis was replaced by a Kriging surrogate model, which was considered to be an uncertain analysis method. The surrogate model was sampled and compared to a database of FEM results to obtain an estimate of the error PDF. The RBDO was carried out using several different RBDO/MDO approaches for comparison. It was found that the deterministic optimization based on the Kriging model was optimistic. The predicted optimum design was subjected to FEM analysis and shown to fail, having a higher maximum stress than the specified limit. Implementing RBDO pushed the deterministic design deeper into feasible design space. It was found that enforcing reliability levels over approximately 1 pushed the predicted optimum solution into feasible design space when evaluated by the FEM analysis.

The wing box problem uncovered another observation: the deterministic solutions predicted using the Kriging model were consistently found to be optimistic - predicting solution locations that were found to be infeasible when tested against the FEM analysis. This was consistently observed regardless of starting location. It might be expected that since the mean errors introduced by the surrogate model were nearly zero, that solutions would be equally likely to fall in feasible design space or infeasible design space. However, since optimizers seek the best possible solution regardless of the limitations of the implemented analysis approaches, it was observed that the solutions were more likely to be optimistic. It is therefore very important to consider the uncertainties introduced by approximate analysis methods in order to protect the design from failure when subjected to better analysis 
methods.

The second case study considered the conceptual design of a regional aircraft with three contributing analysis methods: aerodynamics and stability, performance, and weight and balance. Each contributing analysis package was based partly on the statistically derived empirical equations commonly used in conceptual design. These equations were considered as uncertain analysis methods. Error terms were introduced and modeled probabilistically. This was accomplished by modeling many currently available aircraft designs and predicting the performance characteristics using the low fidelity methods. The predicted performance characteristics were compared with published data to develop PDF functions that model each source of error. It was found that the performance characteristics predicted by the deterministic optimum were considerably better than the reference 737-800 aircraft. However, increasing the reliability level yielded more conservative designs, enhancing the likelihood that the design would comply with all performance targets when subjected to better analysis approaches.

\subsection{Future Work}

Enhancements to this work may include a much more comprehensive consideration aircraft certification requirements, which could be modeled as probabilistic constraints. Better estimates of the characteristics of each source of uncertainty could be obtained by implementing a much larger aircraft specification database. Additionally, implementing high fidelity analysis as a tool for generating data points for assessing the model error associated with the low fidelity equations would enable aircraft conceptual optimization for unconventional designs that are not strictly similar to existing aircraft. Farther enhancements may include the following: 
Sensitivity Analysis Implementing sensitivity analysis on the uncertain errors introduced by approximate analysis methods would enable designers to determine where development and computational should be focused. Reducing the variance of the uncertain error terms may be possible by improving the implemented analysis methods. Determining where improvements would have the most benefit using sensitivity analysis could indicate where additional development and computation time would be best applied.

Certification Aircraft certification requirements include many performance boundaries that new aircraft must be shown to meet or exceed. Handling these constraints in a more comprehensive manner using RBDO could enhance the likelihood that an optimized conceptual design would meet these requirements when subjected to better analysis later in the design process.

High Fidelity Analysis Estimating the error PDFs associated with uncertain analysis methods is limited by the availability of applicable data. A more comprehensive assessment of error may be possible by modeling and comparing the implemented analysis methods with high fidelity analysis results - particularly for component weights and parasite drag prediction.

Data Mining The current research is limited to conventional mid-sized commercial aircraft. As a consequence, the aircraft and engines in the compiled databases were chosen to bracket the intended size and performance of the aircraft designs studied. A more generalized approach may implement a database containing many types of aircraft. In such a case, data mining algorithms may be used to isolate the most relevant aircraft specifications. This would ensure that the predicted performance quantities are not compared with aircraft that are too dissimilar in design and mission. 
Certifiability The certification requirements of commercial aircraft contain many performance attributes that an aircraft must be shown to comply with. Many of these attributes could be quantified and enforced using probabilistic constraints. Using RBDO may improve the likelihood that a conceptual design will comply with certification requirements later in the design process. 


\section{Bibliography}

[1] N. Antoine and I. Kroo, "Framework for aircraft conceptual design and environmental performance studies," AIAA Journal, vol. 43, pp. 2100-2109, Oct. 2005.

[2] R. E. Perez, J. Chung, and K. Behdinan, "Aircraft conceptual design using genetic algorithms," AIAA Paper, vol. 4938, no. 8, 2000.

[3] A. H. W. Bos, "Aircraft conceptual design by genetic/gradient-guided optimization," Engineering Applications of Artificial Intelligence, vol. 11, pp. 377-382, June 1998.

[4] S. Peigin and B. Epstein, "Multiconstrained aerodynamic design of business jet by CFD driven optimization tool," Aerospace Science and Technology, vol. 12, pp. 125134, Mar. 2008.

[5] T. Takemiya, Aerodynamic design applying automatic differentiation and using robust variable fidelity optimization. PhD thesis, Georgia Institute of Technology, 2008.

[6] C. R. Gumbert, G. J. Hou, and P. A. Newman, "High-Fidelity computational optimization for 3-D flexible wings," Optimization and Engineering, vol. 6, pp. 117138, Mar. 2005. 
[7] L. Gonzalez, E. Whitney, K. Srinivas, and J. Periaux, "Optimum multidisciplinary and Multi-Objective wing design in CFD using evolutionary techniques," in Computational Fluid Dynamics 2004, pp. 681-686, 2006.

[8] S. Choi, J. Alonso, I. Kroo, and M. Wintzer, "Multifidelity design optimization of low-boom supersonic sets," Journal of Aircraft, vol. 45, pp. 106-118, Feb. 2008.

[9] J. Kay, W. H. Mason, W. Durham, and F. Lutze, “Aircraft conceptual design,” 1993.

[10] D. Howe, Aircraft conceptual design synthesis. John Wiley \& Sons Inc, 2000.

[11] D. Raymer, Aircraft design : a conceptual approach. Reston VA: American Institute of Aeronautics and Astronautics, 3rd ed. ed., 1999.

[12] E. Torenbeek, Synthesis of subsonic airplane design. Delft Univ. Pr., 1976.

[13] N. E. Antoine, Aircraft optimization for minimal environmental impact. $\mathrm{PhD}$ thesis, Stanford University, 2004. Ph.D.

[14] D. Aronstein and K. Schueler, "Two supersonic business aircraft conceptual designs, with and without sonic boom constraint," Journal of Aircraft, vol. 42, no. 3, pp. 775786, 2005.

[15] A. Striz, B. Kennedy, Z. Siddique, and H. Neeman, "A roadmap for moderate fidelity conceptual design with multilevel analysis and MDO," in Collection of Technical Papers - AIAA/ASME/ASCE/AHS/ASC Structures, Structural Dynamics and Materials Conference, vol. 1, pp. 222-232, 2006.

[16] J. Sobieszczanski-Sobieski and R. T. Haftka, "Multidisciplinary aerospace design optimization: survey of recent developments," Structural and Multidisciplinary $O p$ timization, vol. 14, no. 1, pp. 1-23, 1997. 
[17] A. Giunta, O. Golividov, D. Knill, B. Grossman, W. Mason, L. Watson, and R. Haftka, "Multidisciplinary design optimization of advanced aircraft configurations," in Fifteenth International Conference on Numerical Methods in Fluid Dynamics, pp. 14-34, 1997.

[18] A. Haldar and S. Mahadevan, Probability, reliability and statistical methods in engineering design. Wiley New York, 2000.

[19] W. Oberkampf, S. Deland, B. Rutherford, K. Diegert, and K. Alvin, "Error and uncertainty in modeling and simulation," Reliability Engineering \& System Safety, vol. 75, no. 3, pp. 333-357, 2002.

[20] W. L. Oberkampf, J. C. Helton, C. A. Joslyn, S. F. Wojtkiewicz, and S. Ferson, "Challenge problems: uncertainty in system response given uncertain parameters," Reliability Engineering and System Safety, vol. 85, no. 1-3, pp. 11-19, 2004.

[21] G. W. Parry, “The characterization of uncertainty in probabilistic risk assessments of complex systems," Reliability Engineering \& System Safety, vol. 54, no. 2-3, pp. 119-126, 1996.

[22] Y. T. Wu, H. R. Millwater, and T. A. Cruse, "Advanced probabilistic structural analysis method for implicit performance functions," AIAA journal, vol. 28, no. 9, pp. 1663-1670, 1990.

[23] Y. Tsompanakis, N. D. Lagaros, and M. Papadrakakis, Structural design optimization considering uncertainties. CRC Pr I Llc, 2008.

[24] B. D. Youn, K. K. Choi, L. Du, D. Gorsich, et al., "Integration of possibility-based optimization and robust design for epistemic uncertainty," Journal of Mechanical Design, vol. 129, p. 876, 2007. 
[25] M. E. Pate-Cornel, "Uncertainties in risk analysis: Six levels of treatment," Reliability Engineering \& System Safety, vol. 54, no. 2-3, pp. 95-111, 1999.

[26] S. Mahadevan and R. Rebba, "Inclusion of model errors in Reliability-Based optimization,” Journal of Mechanical Design, vol. 128, pp. 936-944, July 2006.

[27] G. G. Wang and S. Shan, "Review of metamodeling techniques in support of engineering design optimization," Journal of Mechanical Design, vol. 129, p. 370, 2007.

[28] R. Jin, X. Du, and W. Chen, "The use of metamodeling techniques for optimization under uncertainty," Structural and Multidisciplinary Optimization, vol. 25, pp. 99116, July 2003.

[29] B. D. Youn and K. K. Choi, "A new response surface methodology for reliabilitybased design optimization," Computers \& Structures, vol. 82, no. 2-3, pp. 241-256, 2004.

[30] J. Ahn and J. Kwon, "An efficient strategy for reliability-based multidisciplinary design optimization using BLISS," Structural and Multidisciplinary Optimization, vol. 31, pp. 363-372, May 2006.

[31] J. Sobieszczanski-Sobieski, J. S. Agte, and R. R. Sandusky, "Bilevel integrated system synthesis," AIAA Journal, vol. 38, no. 1, pp. 164-172, 2000.

[32] N. Smith and S. Mahadevan, "Probabilistic methods for aerospace system conceptual design," Journal of Spacecraft and Rockets, vol. 40, no. 3, pp. 411-418, 2003.

[33] P. T. Boggs and J. W. Tolle, "Sequential quadratic programming," Acta numerica, vol. 4, pp. 1-51, 2008. 
[34] K. Schittkowski, "The nonlinear programming method of wilson, han and powell with an augmented lagrangian type line search function," Numer. Math, vol. 38, no. 1,1981 .

[35] R. Fletcher, S. Leyffer, D. Ralph, and S. Scholtes, "Local convergence of SQP methods for mathematical programs with equilibrium constraints," SIAM Journal on Optimization, vol. 17, no. 1, pp. 259-286, 2007.

[36] D. E. Goldberg and J. H. Holland, "Genetic algorithms and machine learning," Machine Learning, vol. 3, no. 2, pp. 95-99, 1988.

[37] J. H. Holland, "Genetic algorithms," Scientific American, vol. 267, no. 1, pp. 66-72, 1992.

[38] H. Langer, T. Puehlhofer, and H. Baier, "A multiobjective evolutionary algorithm with integrated response surface functionalities for configuration optimization with discrete variables," Proceedings of the 10th AIAA/ISSMO Multidisciplinary Analysis and Optimization Conference, 2004.

[39] H. Langer, Extended Evolutionary Algorithms for Multiobjective and Discrete Design Optimization of Structures. 2005.

[40] M. Huber, D. Neufeld, J. Chung, H. Baier, and K. Behdinan, "Data mining based mutation function for engineering problems with mixed continuous-discrete design variables," Structural and Multidisciplinary Optimization, vol. 41, pp. 589-604, Apr. 2010.

[41] S. Kodiyalam and J. Sobieszczanski-Sobieski, "Multidisciplinary design optimisation-some formal methods, framework requirements, and application 
to vehicle design," International Journal of Vehicle Design, vol. 25, no. 1, pp. 3-22, 2001.

[42] R. Balling and C. Wilkinson, "Execution of multidisciplinary design optimization approaches on common test problems," AIAA Journal, vol. 35, no. 1, pp. 178-186, 1997.

[43] S. Yi, J. Shin, and G. Park, "Comparison of MDO methods with mathematical examples," Structural and Multidisciplinary Optimization, vol. 35, pp. 391-402, May 2008.

[44] E. J. Cramer, J. E. Dennis Jr, P. D. Frank, R. M. Lewis, and G. R. Shubin, "Problem formulation for multidisciplinary optimization," SIAM Journal on Optimization, vol. 4, p. 754, 1994.

[45] N. M. Alexandrov and S. Kodiyalam, "Initial results of an MDO method evaluation study," AIAA Paper, 1998.

[46] R. E. Perez, H. H. T. Liu, and K. Behdinan, "Evaluation of multidisciplinary optimization approaches for aircraft conceptual design," Proceedings of the 10th AIAA/ISSMO Multidisciplinary Analysis and Optimization Conference, Sept. 2004.

[47] N. Tedford, Comparison of MDO Architectures within a Universal Framework. PhD thesis, University of Toronto, 2006.

[48] N. Brown and J. Olds, "Evaluation of multidisciplinary optimization techniques applied to a reusable launch vehicle," Journal of Spacecraft and Rockets, vol. 43, no. 6, pp. 1289-1300, 2006. 
[49] J. Sobieszczanski-Sobieski, Optimization by decomposition: a step from hierarchic to non-hierarchic systems. Hampton, VA: National Aeronautics and Space Administration, Langley Research Center, 1988.

[50] J. Sobieszczanski-Sobieski, "Sensitivity of complex, internally coupled systems," AIAA journal, vol. 28, no. 1, pp. 153-160, 1990.

[51] M. A. Stelmack and S. M. Batill, "Concurrent subspace optimization of mixed continuous/discrete systems," in Collection of Technical Papers AIAA/ASME/ASCE/AHS/ASC Structures, Structural Dynamics and Materials Conference, vol. 2, pp. 1303-1315, 1997.

[52] R. Tappeta, S. Nagendra, J. E. Renaud, and K. Badhrinath, "Concurrent sub-space optimization (CSSO) MDO algorithm in iSIGHT, CSSO in iSIGHT: validation and testing," GE Research and Development Center, 97CRD186, class, vol. 1, 1998.

[53] R. D. Braun and I. M. Kroo, "Development and application of the collaborative optimization architecture in a multidisciplinary design environment," tech. rep., NASA Langley Technical Report Server, 1995.

[54] R. D. Braun, P. Gage, I. Kroo, and I. Sobieski, "Implementation and performance issues in collaborative optimization," in 6th Annual AIAA/NASA/ISSMO Symposium on Multidisciplinary Analysis and Optimization. AIAA, AIAA, September, 1996.

[55] J. Lin, "Examining and improving collaborative optimization approach to MultiDisciplinary design," in Proceedings of the 9th AIAA/ISSMO Symposium on Multidisciplinary Analysis and Optimization, (Atlanta, Georgia), Sept. 2002.

[56] J. Sobieszczanski-Sobieski, J. S. Agte, and R. R. Sandusky, "Bi-Level integrated system Synthesis(BLISS)," tech. rep., Citeseer, 1998. 
[57] S. Chen, F. Zhang, and M. Khalid, "Evaluation of three decomposition MDO algorithms," ICAS 2002, 2002.

[58] I. Sobieski and I. Kroo, "Aircraft design using collaborative optimization," AIAA, 1996.

[59] R. Perez, H. Liu, and K. Behdinan, "Multidisciplinary optimization framework for control-configuration integration in aircraft conceptual design," Journal of Aircraft, vol. 43, pp. 1937-1948, Dec. 2006.

[60] K. Willcox, "Value-Based multidisciplinary optimization for commercial aircraft design and business risk assessment," Journal of Aircraft, vol. 43, no. 4, pp. 913-921, 2006.

[61] B. Youn, K. Choi, R. Yang, and L. Gu, "Reliability-based design optimization for crashworthiness of vehicle side impact," Structural and Multidisciplinary Optimization, vol. 26, pp. 272-283, Feb. 2004.

[62] J. Hu and Z. Qiu, "Non-probabilistic convex models and interval analysis method for dynamic response of a beam with bounded uncertainty," Applied Mathematical Modelling, vol. 34, pp. 725-734, Mar. 2010.

[63] G. Schueller and H. Jensen, "Computational methods in optimization considering uncertainties - an overview," Computer Methods in Applied Mechanics and Engineering, vol. 198, pp. 2-13, Nov. 2008.

[64] P. Hajela, "Soft computing in multidisciplinary aerospace design - new directions for research,” PROGRESS IN AEROSPACE SCIENCES, vol. 38, no. 1, pp. 1-21, 2002. 
[65] S. Vittal and P. Hajela, "Probabilistic design using empirical distributions," in Collection of Technical Papers - AIAA/ASME/ASCE/AHS/ASC Structures, Structural Dynamics and Materials Conference, vol. 3, pp. 2350-2360, 2003.

[66] G. Q. Cai and I. Elishakoff, "Refined second-order reliability analysis," Structural Safety, vol. 14, no. 4, pp. 267-276, 1994.

[67] M. Allen, M. Raulli, K. Maute, and D. M. Frangopol, "Reliability-based analysis and design optimization of electrostatically actuated MEMS," Computers \& Structures, vol. 82, pp. 1007-1020, May 2004.

[68] B. D. Youn and K. K. Choi, "Selecting probabilistic approaches for ReliabilityBased design optimization," AIAA Journal, vol. 42, no. 1, pp. 124-131, 2004.

[69] H. Agarwal and J. E. Renaud, "Reliability based design optimization using response surfaces in application to multidisciplinary systems," Engineering Optimization, vol. 36, no. 3, p. 291, 2004.

[70] M. Papadrakakis and N. D. Lagaros, "Reliability-based structural optimization using neural networks and monte carlo simulation," Computer Methods in Applied Mechanics and Engineering, vol. 191, pp. 3491-3507, June 2002.

[71] X. Qu and R. T. Haftka, "Design under uncertainty using monte carlo simulation and probabilistic sufficiency factor," in 29th ASME Design Automation Conference, Chicago, IL, USA. Accepted for publication by Journal of Structural and Multidisciplinary Optimization, 2003.

[72] P. Ramu, X. Qu, B. D. Youn, and R. T. Haftka, "Inverse reliability measures and reliability-based design optimisation," International Journal of Reliability and Safety, vol. 1, no. 1, pp. 187-205, 2006. 
[73] W. Liping and R. V. Grandhi, "Efficient safety index calculation for structural reliability analysis," Computers \& Structures, vol. 52, no. 1, pp. 103-111, 1994.

[74] M. Allen and K. Maute, "Reliability-based design optimization of aeroelastic structures," Structural and Multidisciplinary Optimization, vol. 27, no. 4, pp. 228-242, 2004.

[75] B. D. Youn and K. K. Choi, "An investigation of nonlinearity of reliability-based design optimization approaches," ASME Journal of Mechanical Design, vol. 126, 2004.

[76] A. Giunta, M. S. Eldred, L. P. Swiler, T. G. Trucano, and S. F. W. Jr, "Perspectives on optimization under uncertainty: algorithms and applications," AIAA/ISSMO Multidisciplinary analysis and optimization, vol. 10, 2004.

[77] D. Padmanabhan, H. Agarwal, J. Renaud, and S. Batill, "A study using monte carlo simulation for failure probability calculation in Reliability-Based optimization," $O p$ timization and Engineering, vol. 7, no. 3, pp. 297-316, 2006.

[78] M. Gasser and G. I. Schueller, "Reliability-Based optimization of structural systems," Mathematical Methods of Operations Research, vol. 46, pp. 287-307, Oct. 1997.

[79] H. Agarwal, Reliability based design optimization: formulations and methodologies. $\mathrm{PhD}$ thesis, 2004.

[80] M. Rosenblatt, "Remarks on a multivariate transformation," The Annals of Mathematical Statistics, pp. 470-472, 1952.

[81] M. Hohenbichler and R. Rackwitz, "First-order concepts in system reliability," Structural Safety, vol. 1, no. 3, pp. 177-188, 1982. 
[82] K. K. Choi, L. Du, B. D. Youn, and D. Gorsich, "Possibility-based design optimization method for design problems with both statistical and fuzzy input data," 6th World Congresses of Structural and Multidisciplinary Optimization, Rio de Janeiro, Brazil, 2005.

[83] J. R. Red-Horse and A. S. Benjamin, "A probabilistic approach to uncertainty quantification with limited information," Reliability Engineering \& System Safety, vol. 85, pp. 183-190, July.

[84] B. Moller and M. Beer, "Engineering computation under uncertainty - capabilities of non-traditional models," Computers \& Structures, vol. 86, pp. 1024-1041, May 2008.

[85] Y. Ben-Haim, "Convex models of uncertainty: Applications and implications," Erkenntnis, vol. 41, no. 2, pp. 139-156, 1994.

[86] R. E. Moore and F. Bierbaum, Methods and applications of interval analysis. Society for Industrial Mathematics, 1979.

[87] R. E. Moore, "Interval analysis," Englewood Cliffs, New Jersey, 1966.

[88] R. E. Moore and W. Lodwick, "Interval analysis and fuzzy set theory," Fuzzy Sets and Systems, vol. 135, pp. 5-9, Apr. 2003.

[89] L. Du, K. K. Choi, B. D. Youn, and D. Gorsich, "Possibility-based design optimization method for design problems with both statistical and fuzzy input data," Journal of Mechanical Design, vol. 128, p. 928, 2006.

[90] L. Du, K. K. Choi, and B. D. Youn, "Inverse possibility analysis method for Possibility-Based design optimization," AIAA Journal, vol. 44, no. 11, pp. 26822690, 2006. 
[91] T. D. Pham and S. Valliappan, "Constructing the membership function of a fuzzy set with objective and subjective information," Computer-Aided Civil and Infrastructure Engineering, vol. 8, no. 1, pp. 75-82, 2008.

[92] P. Ferrari and M. Savoia, "Fuzzy number theory to obtain conservative results with respect to probability," Computer methods in applied mechanics and engineering, vol. 160, no. 3-4, pp. 205-222, 1998.

[93] M. Huber and H. Baier, "Qualitative knowledge and manufacturing considerations in multidisciplinary structural optimization of hybrid material structures," in Advanced Materials Research, vol. 10, pp. 143-152, 2006.

[94] S. S. Rao and L. Cao, "Optimum design of mechanical systems involving interval parameters," Journal of Mechanical Design, vol. 124, no. 3, pp. 465-472, 2002.

[95] G. Taguchi and A. J. Rafanelli, "Taguchi on robust technology development: Bringing quality engineering upstream," Journal of Electronic Packaging, vol. 116, p. 161, June 1994.

[96] T. Taguchi, T. Yokota, and M. Gen, "Reliability optimal design problem with interval coefficients using hybrid genetic algorithms," Computers \& Industrial Engineering, vol. 35, pp. 373-376, Oct. 1998.

[97] Z. P. Mourelatos and J. Zhou, "Reliability estimation and design with insufficient data based on possibility theory," AIAA Journal, vol. 43, no. 8, p. 1696, 2005.

[98] I. Doltsinis and Z. Kang, "Robust design of structures using optimization methods," Computer Methods in Applied Mechanics and Engineering, vol. 193, pp. 22212237, June 2004. 
[99] B. Chandra and V. Paul, "A robust algorithm for classification using decision trees," in Cybernetics and Intelligent Systems, 2006 IEEE Conference on, pp. 1-5, 2006.

[100] H. Beyer and B. Sendhoff, "Robust optimization - a comprehensive survey," Computer Methods in Applied Mechanics and Engineering, vol. 196, pp. 3190-3218, July 2007.

[101] T. Zou, S. Mahadevan, and R. Rebba, "Computational efficiency in reliability-based optimization," in Proceedings of the 9th ASCE Specialty Conference on Probabilistic Mechanics and Structural Reliability, Albuquerque, NM, 2004.

[102] S. S. Rao, S. S. Rao, and S. S. Rao, Reliability-based design. McGraw-Hill, New York, 1992.

[103] N. Smith, Probabilistic Design of Multidisciplinary Systems. PhD thesis, Vanderbilt University, 2007.

[104] P. B. Thanedar and S. Kodiyalam, "Structural optimization using probabilistic constraints," Structural and Multidisciplinary Optimization, vol. 4, no. 3, pp. 236-240, 1992.

[105] X. Yu, K. K. Choi, and K. H. Chang, "A mixed design approach for probabilistic structural durability," Structural and Multidisciplinary Optimization, vol. 14, pp. 8190, Oct. 1997.

[106] K. Deb, D. Padmanabhan, S. Gupta, and A. Mall, "Reliability-Based multi-objective optimization using evolutionary algorithms," in Evolutionary Multi-Criterion Optimization, pp. 66-80, 2007.

[107] J. Tu, K. K. Choi, and Y. H. Park, "A new study on Reliability-Based design optimization,” Journal of Mechanical Design, vol. 121, pp. 557-564, Dec. 1999. 
[108] B. Youn, K. Choi, and L. Du, "Enriched performance measure approach for reliability-based design optimization," AIAA Journal, vol. 43, no. 4, pp. 874-884, 2005.

[109] M. S. Eldred, H. Agarwal, V. M. Perez, S. F. Wojtkiewicz, and J. E. Renaud, "Investigation of reliability method formulations in DAKOTA/UQ," Structure and Infrastructure Engineering, vol. 3, no. 3, pp. 199-224, 2007.

[110] S. Shan and G. G. Wang, "Reliable design space and complete single-loop reliability-based design optimization," Reliability Engineering \& System Safety, vol. 93, pp. 1218-1230, Aug. 2008.

[111] X. Du and W. Chen, "Sequential optimization and reliability assessment method for efficient probabilistic design," Transactions-American Society of Mechanical Engineers Journal of Mechanical Design, pp. 225-233, 2004.

[112] Y. T. Wu, Y. Shin, R. H. Sues, and M. A. Cesare, "Safety-factor based approach for probability-based design optimization," in AIAA/ASME/ASCE/AHS/ASC Structures, Structural Dynamics, and Materials Conference and Exhibit, 42 nd, Seattle, WA, 2001.

[113] Y. T. Wu and W. Wang, "Efficient probabilistic design by converting reliability constraints to approximately equivalent deterministic constraints," Journal of Integrated Design and Process Sciences, vol. 2, no. 4, pp. 13-21, 1998.

[114] X. Chen, T. K. Hasselman, and D. J. Neill, "Reliability based structural design optimization for practical applications," in Proceedings of the 38th AIAA/ASME/ASCE/AHS/ASC Structures, Structural Dynamics, and Materials Conference, pp. 2724-2732, 1997. 
[115] N. Kuschel and R. Rackwitz, "Optimal design under time-variant reliability constraints," Structural Safety, vol. 22, no. 2, pp. 113-127, 2000.

[116] J. Liang, Z. P. Mourelatos, and J. Tu, "A single-loop method for reliability-based design optimisation," International Journal of Product Development, vol. 5, no. 1, pp. 76-92, 2008.

[117] B. M. Adams, K. R. Dalbey, M. S. Eldred, D. M. Gay, L. P. Swiler, W. J. Bohnhoff, J. P. Eddy, K. Haskell, and P. D. Hough, "DAKOTA, a multilevel parallel Object-Oriented framework for design optimization, parameter estimation, uncertainty quantification, and sensitivity analysis," 2006.

[118] M. S. Eldred, B. J. Bichon, and B. M. Adams, "Overview of reliability analysis and design capabilities in DAKOTA,"

[119] M. S. Eldred, A. A. Giunta, S. F. W. Jr, and T. G. Trucano, "Formulations for surrogate-based optimization under uncertainty," in Proceedings of the 9th AIAA/ISSMO Symposium on Multidisciplinary Analysis and Optimization, 2002.

[120] Y. Wu, Y. Shin, R. H. Sues, and M. A. Cesare, "Probabilistic function evaluation system (ProFES) for reliability-based design," Structural Safety, vol. 28, no. 1-2, pp. 164-195, 2006.

[121] D. Neufeld and J. Chung, "Unmanned aerial vehicle conceptual design using a genetic algorithm and data mining," in Proceedings of the Infotech@Aerospace Conference, Sept. 2005.

[122] D. Neufeld and J. Chung, "Enhancing UAV conceptual design using evolutionary algorithms and data mining," in Proceedings of the 2007 ICCSA Conference, 2007. 
[123] D. Neufeld and J. Chung, "Conceptual design optimization of very light jets," Proceedings of the 2007 CANCAM Conference, June 2007.

[124] D. Neufeld, J. Chung, and K. Behdinan, "Development of a flexible MDO architecture for aircraft conceptual design," in Proceedings of the 2008 EngOpt conference. Rio de Jenario, Brazil, 2008.

[125] D. Neufeld, J. Chung, and K. Behdinan, "An approach to Multi-Objective aircraft design," Future Application and Middleware Technology on e-Science, pp. 103-112, 2009.

[126] The Mathworks, "MATLAB (R),” 2009.

[127] A. Mullur, P. Hajela, and Y. Bahei-El-Din, "Uncertainty management in design optimization of coupled systems," in Collection of Technical Papers - 11th AIAA/ISSMO Multidisciplinary Analysis and Optimization Conference, vol. 3, pp. 1540-1546, 2006.

[128] N. Smith, Probabilistic design of multidisciplinary systems. PhD thesis, Vanderbilt University, 2007. Ph.D.

[129] R. D. Braun, A. A. Moore, and I. M. Kroo, "Use of the collaborative optimization architecture for launch vehicle design," in NASA Langley Research Center, Proceedings of the 6 thAIAA/USAF/NASA/ISSMO Symposium on Multidisciplinary Analysis and Optimization, AIAA Paper, 1996.

[130] A. Chiralaksanakul and S. Mahadevan, "First-Order approximation methods in Reliability-Based design optimization," Journal of Mechanical Design, vol. 127, no. 5 , p. $851,2005$. 
[131] E. Salajegheh and G. N. Vanderplaats, "Optimum design of trusses with discrete sizing and shape variables," Structural and Multidisciplinary Optimization, vol. 6, pp. 79-85, June 1993.

[132] R. Jin, W. Chen, and T. Simpson, "Comparative studies of metamodelling techniques under multiple modelling criteria," Structural and Multidisciplinary Optimization, vol. 23, pp. 1-13, Dec. 2001.

[133] T. Simpson, J. Poplinski, P. N. Koch, and J. Allen, "Metamodels for computer-based engineering design: Survey and recommendations," Engineering with Computers, vol. 17, pp. 129-150, July 2001.

[134] T. W. Simpson, T. M. Mauery, J. J. Korte, and F. Mistree, "Kriging models for global approximation in simulation-based multidisciplinary design optimization,” AIAA journal, vol. 39, no. 12, pp. 2233-2241, 2001.

[135] G. Venter, R. T. Haftka, and J. H. Starnes, "Construction of response surface approximations for design optimization," AIAA Journal, vol. 36, pp. 2242-2249, 1998.

[136] W. J. Roux, N. Stander, and R. T. Haftka, "Response surface approximations for structural optimization," International Journal for Numerical Methods in Engineering, vol. 42, no. 3, pp. 517-534, 1998.

[137] J. Roskam, Airplane design. DARcorporation, 2000.

[138] P. Konstadinopoulos, D. Thrasher, A. Nayfeh, L. Watson, and D. Mook, "VortexLattice method for general, unsteady aerodynamics," Journal of Aircraft, vol. 22, no. 1, pp. 43-49, 1985.

[139] M. Drela and H. Youngren, "Athena vortex lattice," Software Package, Ver. 3.27, 2008. 
[140] The Boeing Company, "Airplane characteristics for airport planning." http://www.boeing.com/commercial/airports/plan_manuals.html, 2010.

[141] E. Torenbeek, "Quick estimation of wing structural weight for preliminary aircraft design," Aircraft Engineering and Aerospace Technology: An International Journal, vol. 44, pp. 18-19, 1993.

[142] C. Svoboda, "Turbofan engine database as a preliminary design tool," Aircraft Design, vol. 3, no. 1, pp. 17-31, 2000.

[143] R. Y. K. Fung, J. Tang, and D. Wang, "Extension of a hybrid genetic algorithm for nonlinear programming problems with equality and inequality constraints," Computers \& Operations Research, vol. 29, pp. 261-274, Mar. 2002.

[144] A. A. Giunta, J. M. McFarland, L. P. Swiler, and M. S. Eldred, "The promise and peril of uncertainty quantification using response surface approximations," Structure and Infrastructure Engineering, vol. 2, no. 3, pp. 175-189, 2006.

[145] Federal Aviation Administration, "Federal aviation regulations." http://rgl.faa.gov/Regulatory_and_Guidance_Library/. 
Appendix A

\section{Data Sources}


Table A.1: Aircraft Specification Database

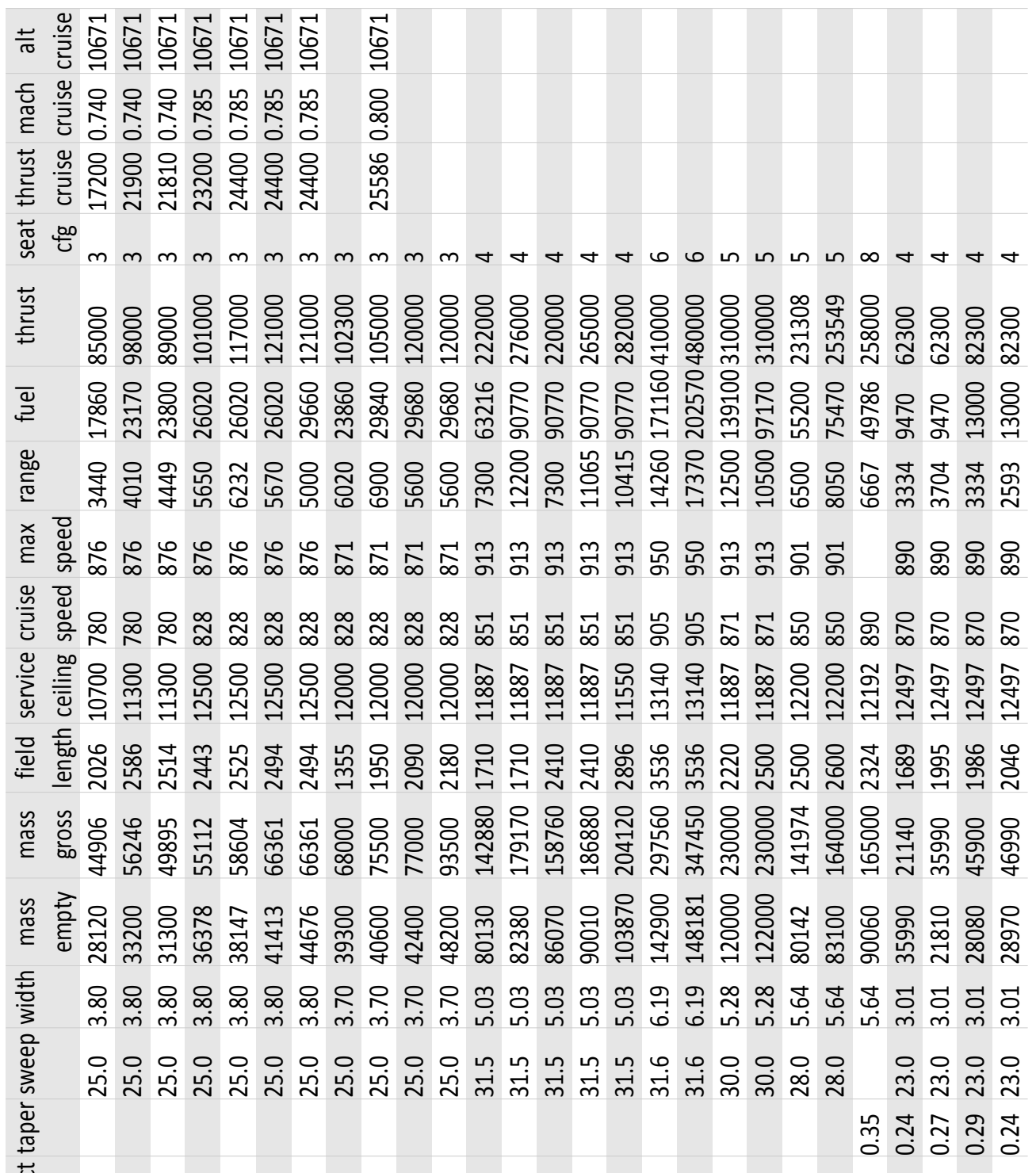

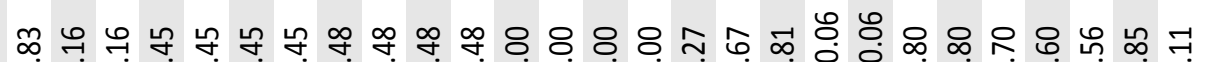

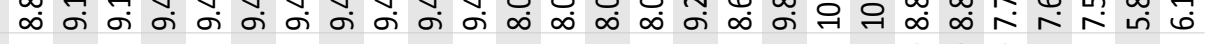

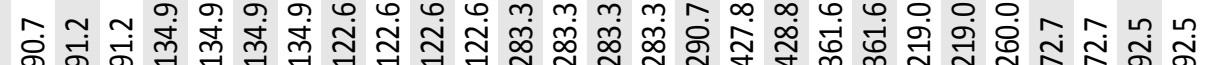

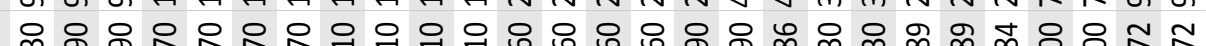
ஸे ஹं

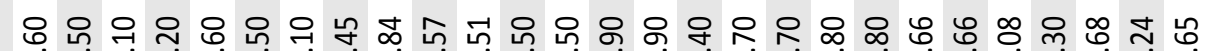
ஸे

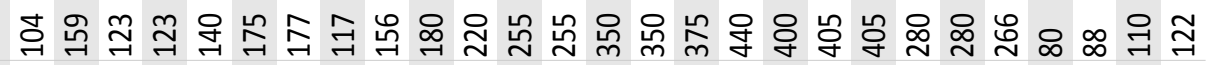

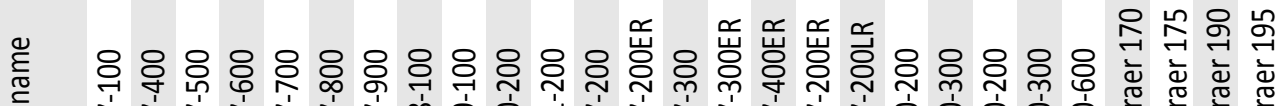
Th

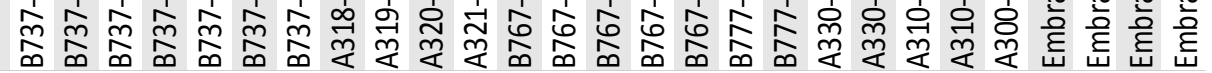


Table A.2: Engine Performance Database

\begin{tabular}{|c|c|c|c|c|c|c|c|c|c|c|c|c|}
\hline Number & Tsl & hcr & Mcr & $\mathrm{Tcr}$ & SFCto & SFCcr & Number & Tsl & hcr & Mcr & Tcr & SFCto \\
\hline 1 & 6672 & 9144 & 0.70 & 2669 & 0.456 & 0.750 & 38 & 133447 & 710668 & 0.80 & 25586 & 0.369 \\
\hline 2 & 8452 & 11000 & 0.70 & 2251 & 0.475 & 0.758 & 39 & 146791 & 10671 & 0.80 & 25466 & 0.370 \\
\hline 3 & 13545 & 12195 & 0.80 & 2860 & 0.560 & 0.540 & 40 & 151240 & 10668 & 0.80 & 31582 & 0.361 \\
\hline 4 & 14710 & 6000 & 0.48 & 4341 & 0.570 & 0.795 & 41 & 156911 & 11000 & 0.80 & 34322 & 0.359 \\
\hline 5 & 16458 & 12192 & 0.80 & 3634 & 0.447 & 0.835 & 42 & 166363 & 310668 & 0.80 & 37601 & 0.355 \\
\hline 6 & 20017 & 12192 & 0.80 & 4386 & 0.443 & 0.771 & 43 & 170144 & 1067 & 0.85 & 28913 & 0.330 \\
\hline 7 & 21129 & 12192 & 0.80 & 4680 & 0.442 & 0.756 & 44 & 170589 & 10668 & 0.85 & 28913 & 0.330 \\
\hline 8 & 21129 & 12192 & 0.80 & 4951 & 0.442 & 0.675 & 45 & 177929 & 10668 & 0.85 & 40568 & 0.348 \\
\hline 9 & 22241 & 12192 & 0.80 & 4982 & 0.441 & 0.679 & 46 & 186323 & 31100 & 0.80 & 37263 & 0.286 \\
\hline 10 & 23424 & 12195 & 0.80 & 4951 & 0.390 & 0.680 & 47 & 191718 & 310671 & 0.80 & 37788 & 0.610 \\
\hline 11 & 24198 & 12192 & 0.80 & 4693 & 0.503 & 0.830 & 48 & 191718 & 3106 & 0.80 & 38700 & 0.345 \\
\hline 12 & 25355 & 12192 & 0.80 & 5872 & 0.394 & 0.679 & 49 & 193942 & 10668 & 0.85 & 45372 & 0.344 \\
\hline 13 & 25466 & 12192 & 0.80 & 6512 & 0.372 & 0.640 & 50 & 222 & 110 & 0.85 & 46604 & 0.344 \\
\hline 14 & 26325 & 12195 & 0.80 & 5827 & 0.370 & 0.650 & 51 & 222411 & 10668 & 0.85 & 48930 & 0.333 \\
\hline 15 & 30203 & 7620 & 0.70 & 10008 & 0.408 & 0.721 & 52 & 229 & 511 & 0.75 & 47667 & 0.360 \\
\hline 16 & 31004 & 7622 & 0.70 & 10008 & 0.410 & 0.720 & 53 & 233532 & 10 & 0.80 & 41813 & 0.311 \\
\hline 17 & 44037 & 7622 & 0.74 & 16592 & 0.560 & 0.800 & 54 & 233532 & $10 t$ & 0.85 & 48041 & 0.390 \\
\hline 18 & 50011 & 7620 & 0.75 & 13238 & 0.374 & 0.680 & 55 & 233532 & 106 & 0.80 & 53379 & 0.320 \\
\hline 19 & 61608 & 10671 & 0.80 & 11343 & 0.430 & 0.690 & 56 & 2357 & 510 & 0.85 & 53156 & 0.329 \\
\hline 20 & 63743 & 8001 & 0.75 & 15689 & 0.360 & 0.650 & 57 & 249100 & 10668 & 0.85 & 54491 & 0.324 \\
\hline 21 & 65834 & 12497 & 0.80 & 10231 & 0.405 & 0.630 & 58 & 2542 & 10 & 0.80 & 50398 & 0.316 \\
\hline 22 & 66034 & 10671 & 0.80 & 15480 & 0.390 & 0.640 & 59 & 257543 & 310 & 0.80 & 53565 & 0.329 \\
\hline 23 & 68503 & 10671 & 0.80 & 13122 & 0.450 & 0.690 & 60 & 257997 & 710671 & 0.80 & 57026 & 0.320 \\
\hline 24 & 80415 & 11000 & 0.75 & 15297 & 0.396 & 0.610 & 61 & 266893 & 3106 & 0.80 & 49131 & 0.350 \\
\hline 25 & 88444 & 10671 & 0.80 & 19483 & 0.370 & 0.620 & 62 & 267294 & 10668 & 0.85 & 50398 & 0.322 \\
\hline 26 & 90223 & 11000 & 0.80 & 19319 & 0.390 & 0.630 & 63 & 269562 & 10671 & 0.85 & 52547 & 0.560 \\
\hline 27 & 97861 & 10668 & 0.76 & 16014 & 0.386 & 0.610 & 64 & 269562 & 10668 & 0.85 & 52547 & 0.318 \\
\hline 28 & 97861 & 10668 & 0.85 & 22103 & 0.386 & 0.657 & 65 & 272676 & 1067 & 0.80 & 50443 & 0.340 \\
\hline 29 & 97861 & 10668 & 0.85 & 22419 & 0.386 & 0.655 & 66 & 300255 & 510668 & 0.82 & 51155 & 0.308 \\
\hline 30 & 97861 & 10671 & 0.80 & 23064 & 0.340 & 0.570 & 67 & 334284 & 10668 & 0.82 & 51155 & 0.298 \\
\hline 31 & 107869 & 11000 & 0.80 & 26970 & 0.490 & 0.700 & 68 & 346516 & 510668 & 0.83 & 57827 & 0.295 \\
\hline 32 & 111206 & 10671 & 0.80 & 22552 & 0.350 & 0.580 & 69 & 376320 & 10668 & 0.80 & 62119 & 0.286 \\
\hline 33 & 111206 & 10671 & 0.80 & 25680 & 0.360 & 0.570 & 70 & 394477 & 710668 & 0.85 & 107131 & 0.274 \\
\hline 34 & 117433 & 10668 & 0.80 & 24376 & 0.376 & 0.644 & 71 & 401230 & 10668 & 0.80 & 81847 & 0.280 \\
\hline
\end{tabular}


Table A.3: Wing Box Database

\begin{tabular}{|c|c|c|c|c|c|c|c|c|c|c|c|}
\hline $\mathrm{X1}$ & $x 2$ & X3 & $\mathrm{X4}$ & X5 & X6 & X7 & $\mathrm{X8}$ & $\mathrm{X9}$ & & & \\
\hline & & & & & & & & & & & \\
\hline & & & & & & & & & & & \\
\hline & & & & & & & & & & & \\
\hline & & & & & & & & & & & \\
\hline & & & & & & & & & & & \\
\hline & & & & & & & & & & & \\
\hline & 255 & & 00 & 0070 & & & & & & & \\
\hline 02 & 232 & 1 & 0047 & 70 & 1 & 65 & & & & & \\
\hline רחמת & & & 2 & 75 & & & & & & & \\
\hline 029 & 0202 & 0252 & .00 & 0058 & & & & & & & \\
\hline 0037 & 0231 & 7 & 0.0048 & 0.0060 & & 8 & & 80 & & & \\
\hline 0.0025 & 0260 & 8 & 0 & 0 & & & & & & & \\
\hline & & & & & & & & & & & \\
\hline & & & & & & & & & & & \\
\hline 000 & & & & & & & & & & & \\
\hline & & & & & & & & & & & \\
\hline & & & & & & & & & & & \\
\hline & & & & & & & & & & & \\
\hline & & & & & & & & & & & \\
\hline & & & & & & & & & & & \\
\hline & & & & & & & & & & & \\
\hline & & & & & & & & & & & \\
\hline & & & & & & & & & & & \\
\hline & & & 0 & & & & & & & & \\
\hline & & & & & & & & & & & \\
\hline & & & & & & & & & & & \\
\hline & & & & & & & & & & & \\
\hline & & & & & & & & & & & \\
\hline 00020 & 0228 & 8 & & 0060 & & 0 & & & & & \\
\hline & & & & & & & & & & & \\
\hline & & & & & & & & & & & \\
\hline & & & & & & & & & & & \\
\hline & & & & & & & & & & & \\
\hline & & & & & & & & & & & \\
\hline & & & & & & & & & & & \\
\hline & & & & & & & & & & & \\
\hline & & & & & & & & & & & \\
\hline & & & & & & & & & & & \\
\hline & & & & & & & & & & & \\
\hline .0052 & 0230 & 0201 & 0.0038 & 0.0070 & .0193 & 0.0249 & 12.0050 & .6650 & 0.0515 & $0.044^{2}$ & \\
\hline
\end{tabular}


Table A.4: Wing Box Database (cont)

\begin{tabular}{|c|c|c|c|c|c|c|c|c|c|c|c|}
\hline 040 & 165 & 205 & 059 & 089 & & & & & & & \\
\hline 038 & 258 & 149 & 0039 & 79 & 1 & & & & & & \\
\hline 0039 & 0173 & 9 & 0058 & & & & & & & & \\
\hline 0.0038 & & & & & & & & & & & \\
\hline & & & & & & & & & & & \\
\hline & & & & & & & & & & & \\
\hline & & & & & & & & & & & \\
\hline & & & & & & & & & & & \\
\hline & & & & & & & & & & & \\
\hline & & & & & & & & & & & \\
\hline & & & & & & & & & & & \\
\hline 0037 & & & & & & & & & & & 136 \\
\hline 03 & & & & & 0 & & & & & & 18 \\
\hline 02 & & & & & & & & & & & \\
\hline & & & & & & & & & & & \\
\hline & & & & & & & & & & & \\
\hline 02 & & & & & & & & & & & 137 \\
\hline 0026 & & & & & & & & & & & \\
\hline 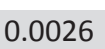 & & & & & & & & & & & \\
\hline & & & & & & & & & & & \\
\hline & & & & & & & & & & & \\
\hline & & & & & & & & & & & \\
\hline & & & & & & & & & & & \\
\hline & & & & & & & & & & & \\
\hline & & & & & & & & & & & \\
\hline & & & & & & & & & & & \\
\hline & & & & & & & & & & & \\
\hline & & & & & & & & & & & \\
\hline & & & & & & & & & & & \\
\hline & & & & & & & & & & & \\
\hline & & & & & & & & & & & \\
\hline 035 & & & & & & & & & & & 78 \\
\hline & & & & & & & & & & & 9930 \\
\hline & & & & & & & & & & & \\
\hline & & & & & & & & & & & 91 \\
\hline 0. & & & 0054 & & & & & & & & \\
\hline 0.003 & . & & 005 & & & & & 00 & & & 00 \\
\hline & & & & & & & & & & & \\
\hline & & & & & & & & & & & \\
\hline & & & & & & & & & & & \\
\hline 0.0039 & 0.0184 & 0.0234 & 0.0052 & 0.0064 & 0.0224 & 0.0189 & & 8.0770 & 0.1860 & 0.1765 & 0. \\
\hline
\end{tabular}


Table A.5: Wing Box Database (cont)

\begin{tabular}{|c|c|c|c|c|c|c|c|c|c|c|c|}
\hline 33 & 0.0209 & 0202 & 0.0056 & .0049 & 0.0152 & 0.0154 & 12.6870 & 660 & 3578 & 966 & 452 \\
\hline 0041 & 0.0239 & .0255 & .0036 & .0064 & 0.0260 & 0.0157 & 11.2540 & 9.7480 & 0.5843 & 0.6366 & 1.0895 \\
\hline 0.0031 & 0.0174 & .0246 & .0050 & .0066 & 0.0175 & 0.0216 & 10.4770 & 7.6100 & 0.6441 & 0.6983 & 1.0841 \\
\hline 0.0038 & 0.0175 & 0.0145 & 0.0038 & 0.0066 & 0.0236 & 0.0161 & 7.4720 & 10.6450 & 0.1705 & 0.1942 & 1.1392 \\
\hline 0.0035 & 0.0242 & 0.0161 & 0.0056 & 0.0057 & 0.0237 & 0.0228 & 11.6230 & 10.6100 & 0.4364 & 0.4667 & 1.0694 \\
\hline 0.0027 & 0.0153 & 0.0146 & 0.0051 & 0.0056 & 0.0194 & 0.0249 & 8.8680 & 6.9960 & 0.5105 & 0.5261 & 1.0305 \\
\hline 0.0027 & 0.0186 & 0.0209 & 0.0039 & 0.0060 & 0.0149 & 0.0234 & 8.4710 & 10.2110 & 0.2775 & 0.2814 & 1.0139 \\
\hline 0.0025 & 0.0251 & 0.0213 & 0.0033 & 0.0082 & 0.0234 & 0.0229 & 7.4740 & 7.6670 & 0.1858 & 0.2326 & 1.2520 \\
\hline 0.0034 & 0.0170 & 0.0226 & 0.0046 & 0.0091 & 0.0254 & 0.0155 & 11.6940 & 7.4270 & 0.9928 & 0.8812 & 0.8876 \\
\hline 0.0023 & 0.0172 & 0.0182 & 0.0043 & 0.0052 & 0.0169 & 0.0158 & 8.8220 & 7.3750 & 0.4900 & 0.4274 & 0.8723 \\
\hline 0.0043 & 0.0165 & 0.0180 & 0.0045 & 0.0051 & 0.0181 & 0.0155 & 12.2860 & 5.9670 & 1.7619 & 1.9245 & 1.0923 \\
\hline 0.0040 & 0.0223 & 0.0163 & 0.0041 & 0.0086 & 0.0198 & 0.0200 & 11.4880 & 10.8710 & 0.4288 & 0.4333 & 1.0104 \\
\hline 0.0028 & 0.0198 & 0.0205 & 0.0046 & 0.0070 & 0.0171 & 82 & 8.4 & 50 & 261 & 83 & 1.0231 \\
\hline 0.0023 & 0.0268 & 0.0166 & 0.0038 & 0.0060 & 0.0198 & 0.0205 & 10 & 70 & 561 & 921 & 1.3819 \\
\hline 0.0031 & 0.0264 & 0.0 & 33 & 0.0054 & 31 & 76 & 80 & 20 & 65 & 88 & 1.0784 \\
\hline 0.0041 & 0.0208 & 0.0170 & 0.0042 & 0.0084 & 0.0244 & 0.0171 & 7.7780 & 9.5900 & 0.2080 & 189 & 1.0522 \\
\hline 0.0027 & 0.0270 & 0.0221 & 0.0053 & 0.0083 & 0.0238 & 0.0161 & 8.4780 & 7.3580 & 0.4445 & 0.4602 & 1.0354 \\
\hline 0.0033 & 0.0196 & 0.0262 & 0.0060 & 0.0068 & 0.0258 & 0.0271 & 12.6660 & 9.5020 & 0.5740 & 0.6425 & 1.1194 \\
\hline 0.0030 & 0.0161 & 0.0 & 51 & 0.0062 & 0.0208 & 48 & 20 & 30 & 526 & 06 & 511 \\
\hline & & & & & & & & & & & 154 \\
\hline 0.0035 & 0.0215 & 0.0246 & 0.0042 & 0.0071 & 0.0231 & 0.0225 & 9.2570 & 8.1160 & 0.3186 & 0.3644 & 1.1438 \\
\hline 0.0041 & 0.0202 & 0.0167 & 0.0058 & 0.0090 & 0.0222 & 0.0205 & 7.5480 & 6.7330 & 0.2907 & 0.2932 & 1.0087 \\
\hline 0.0035 & 0.0260 & 0.0213 & 0.0038 & 0.0084 & 0.0204 & 0.0220 & 12.8900 & 8.6620 & 0.7716 & 0.6941 & 0.8996 \\
\hline 0.0028 & 0.0190 & 0.0216 & 0.0041 & 0.0071 & 0.0208 & 0.0166 & 10.9 & 20 & 0.8404 & 59 & 1.0660 \\
\hline 0.0037 & 0.02 & 0.0 & 0.0 & 1 & 2 & 2 & 10. & 20 & 52 & 79 & 1.3226 \\
\hline & 0.0239 & 0.0 & 0.0050 & 0.0088 & 10 & 81 & 11.2430 & 710 & 84 & 22 & 0.8807 \\
\hline 0.0036 & 0.0191 & 0.0202 & 0.0040 & 0.0086 & 0.0219 & 0.0232 & 8.4490 & 8.4240 & 0.2630 & 0.2540 & 0.9657 \\
\hline 0.0028 & 0.0184 & 0.0213 & 0.0048 & 0.0056 & 0.0258 & 0.0230 & 11.3790 & 7.1550 & 0.7775 & 0.7585 & 0.9756 \\
\hline 0.0031 & 0.0232 & 0.0236 & 0.0051 & 0.0058 & 0.0252 & 0.0222 & 8.2850 & 8.2380 & 0.2192 & 0.2219 & 1.0122 \\
\hline 0.0029 & 0.0210 & 0.0227 & 0.0051 & 0.0076 & 0.0198 & 0.0197 & 7.1140 & 10.8450 & 0.1167 & 0.1047 & 0.8974 \\
\hline 0.0027 & 0.0228 & 0.0159 & 0.0044 & 0.0085 & 0.0222 & 0.0233 & 10.7080 & 10.0390 & 0.3906 & 0.3577 & 0.9158 \\
\hline 0.0034 & 0.0208 & 0.0198 & 0.0047 & 0.0067 & 0.0240 & 0.0156 & 11.8410 & 9.2310 & 0.7370 & 0.6491 & 0.8807 \\
\hline 0.0035 & 0.0207 & 0.0186 & 0.0042 & 0.0084 & 0.0193 & 0.0199 & 10.8080 & 7.3390 & 0.6842 & 0.7042 & 1.0293 \\
\hline 0.0038 & 0.0235 & 0.0180 & 0.0055 & 0.0074 & 0.0174 & 0.0236 & 11.6490 & 10.8370 & 0.4833 & 0.4822 & 0.9978 \\
\hline 0.0027 & 0.0189 & 0.0266 & 0.0061 & 0.0056 & 0.0149 & 0.0204 & 9.7840 & 10.2280 & 0.3961 & 0.3983 & 1.0056 \\
\hline 0.0024 & 0.0256 & 0.0250 & 0.0053 & 0.0081 & 0.0197 & 0.0265 & 9.8030 & 6.7300 & 0.5705 & 0.5989 & 1.0497 \\
\hline 0.0027 & 0.0156 & 0.0225 & 0.0043 & 0.0066 & 0.0243 & 0.0194 & 8.7740 & 10.5190 & 0.2410 & 0.2433 & 1.0096 \\
\hline 0.0031 & 0.0264 & 0.0208 & 0.0042 & 0.0065 & 0.0166 & 0.0254 & 8.6850 & 9.8070 & 0.2903 & 0.2680 & 0.9233 \\
\hline 0.0039 & 0.0257 & 0.0244 & 0.0042 & 0.0089 & 0.0234 & 0.0179 & 12.0700 & 10.9330 & 0.4977 & 0.4102 & 0.8241 \\
\hline 0.0038 & 0.0190 & 0.0154 & 0.0053 & 0.0053 & 0.0195 & 0.0214 & 7.8780 & 8.8490 & 0.2169 & 0.2130 & 0.9822 \\
\hline 0.0038 & 0.0233 & 0.0156 & 0.0044 & 0.0061 & 0.0218 & 0.0177 & 10.8930 & 5.9470 & 1.1764 & 1.4104 & 1.1989 \\
\hline
\end{tabular}


Table A.6: Wing Box Database (cont)

\begin{tabular}{|c|c|c|c|c|c|c|c|c|c|c|c|}
\hline 025 & 0.0156 & 221 & .0045 & 0053 & .0169 & 0.0163 & 11.9140 & טדנס & 1.6117 & 1.5590 & נוס \\
\hline 036 & 0153 & 0222 & 0058 & .0073 & 0194 & 0.0205 & 9.5920 & 8.1390 & 0.4860 & 5079 & 0451 \\
\hline .0040 & 0.0178 & 0.0234 & .0046 & .0058 & 0.0158 & 0.0215 & 12.1360 & .1260 & 0.9596 & .9062 & 0.9443 \\
\hline 0036 & 0.0148 & 0.0245 & .0049 & .0086 & 0.0181 & 0.0267 & 12.3360 & 8.1950 & 0.9779 & 0.9734 & 0.9954 \\
\hline 0.0031 & 0.0193 & 0.0264 & 0.0037 & 0.0056 & 0.0231 & 0.0157 & 8.1410 & 10.8630 & 0.2094 & 2194 & .0476 \\
\hline 0.0034 & 0.0225 & 0.0150 & 0.0041 & 0.0058 & 0.0250 & 0.0177 & 7.5350 & 9.2790 & 1998 & 1805 & 0.9034 \\
\hline .0035 & 0.0150 & 0.0199 & 0.0046 & 0.0079 & 0.0194 & 0.0173 & 8.0270 & 10.0120 & 285 & 84 & 9560 \\
\hline 32 & 0.0 & 38 & 61 & 2 & 3 & & 20 & & & 5 & 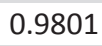 \\
\hline 0.0023 & 0.0214 & 88 & 55 & 35 & 47 & 67 & 90 & 30 & 48 & 62 & 1.1075 \\
\hline 0.0034 & 0.0215 & 0.0 & 38 & 0.0 & 0.0 & 49 & 40 & 60 & 75 & 03 & 560 \\
\hline 0.0034 & 0.0175 & 0.0190 & 0.0034 & 0.0059 & 0.0229 & 0.0268 & 11.7370 & 6.6660 & 1.0008 & 0.9781 & 0.9773 \\
\hline 0.0038 & 0.0190 & 0.0154 & 0.0053 & 0.0053 & 0.0195 & 0.0214 & 7.8780 & 8.8490 & 0.2169 & 0.2222 & 1.0243 \\
\hline 0.0033 & 0.0215 & 0.0237 & 0.0033 & 0.0074 & 0.0255 & 0.0196 & 11.1990 & 8.6100 & 95 & 0.5655 & 1.0679 \\
\hline 40 & 2 & 74 & & & 4 & & 0 & 30 & & & 99 \\
\hline & & & & & & & & & & & 1.0727 \\
\hline 0.0034 & 0.0190 & 0.0184 & 0.0040 & 0.0078 & 0.0175 & 0.0241 & 7.3550 & 7.6210 & 0.2468 & 0.2628 & 1.0649 \\
\hline 0.0025 & 0.0188 & 0.0168 & 0.0049 & 0.0062 & 0.0196 & 0.0155 & 12.7540 & 9.3130 & 0.9094 & 0.9080 & 0.9985 \\
\hline 0.0040 & 0.0261 & 0.0174 & 0.0039 & 0.0081 & 0.0248 & 0.0202 & 7.2240 & 10.2510 & 0.1325 & 0.1554 & 1.1729 \\
\hline 0.0042 & 0.0262 & 0.0215 & 0.0041 & 0.0071 & 0.0160 & 85 & 12.0440 & 8.6140 & 331 & 0.7547 & 0.8546 \\
\hline 0.0038 & 0.0216 & 0.0178 & 0.0052 & 0.0089 & 0.0158 & 0.0220 & 9.6940 & 10.2540 & 36 & 0.3597 & 0.9629 \\
\hline 0.0029 & 0.0236 & 0.0244 & 0.0043 & 0.0051 & .0236 & 0.0165 & 10.7100 & 7.4780 & 0.7 & 0.6824 & 0.9015 \\
\hline 0.0030 & 0.0221 & 0.0268 & 0.0045 & 0.0077 & 0.0229 & 0.0270 & 11.2290 & 10.5030 & 0.3295 & 0.3951 & 1.1991 \\
\hline 0.0028 & 0.0237 & 0.0223 & 0.0054 & 0.0082 & 0.0263 & 0.0241 & 11.1060 & 8.9920 & 0.3962 & 0.4250 & 1.0727 \\
\hline 0.0032 & 0.0160 & 0.0261 & 0.0048 & 0.0053 & 0.0260 & 0.0265 & 9.8110 & 10.9640 & 0.2633 & 0.2772 & 1.0528 \\
\hline 0.0040 & 0.0166 & 0.0156 & 0.0039 & 0.0091 & 0.0179 & 0.0202 & 9.9100 & 10.2550 & 0.3504 & 0.3502 & 0.9993 \\
\hline 0.0025 & 0.0165 & 0.0227 & 0.0034 & 0.0086 & 0.0205 & 0.0207 & 12.8810 & 10.9760 & 0.5997 & 0.5376 & 0.8964 \\
\hline
\end{tabular}




\section{Appendix B}

\section{Aviation Regulations}

The aviation regulations were accessed from the Federal Aviation Administration (FAA) database [145].

Table B.1: Regulations for Fuselage Sizing

$\nabla$ Sec. 25.815

Part 25 AIRWORTHINESS STANDARDS: TRANSPORT CATEGORY AIRPLANES

\begin{tabular}{|l|ll|}
\hline Subpart D--Design and Construction & Emergency Provisions \\
\hline
\end{tabular}

Sec. 25.815

Width of aisle.

The passenger aisle width at any point between seats must equal or exceed the values in the following table:

\begin{tabular}{|c|c|c|}
\hline \multirow[t]{2}{*}{ Passenger seating capacity } & \multicolumn{2}{|c|}{ Minimum passenger aisle width (inches) } \\
\hline & $\begin{array}{l}\text { Less than } 25 \text { inches } \\
\text { from floor }\end{array}$ & $\begin{array}{l}25 \text { inches and more } \\
\text { from floor }\end{array}$ \\
\hline 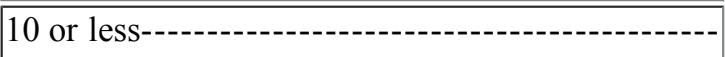 & ${ }^{1} 12$ & 15 \\
\hline 11 through 19---o- & 12 & 20 \\
\hline 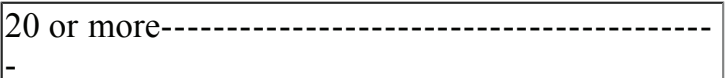 & 15 & 20 \\
\hline
\end{tabular}


Table B.2: Regulations for Fuselage Sizing (continued)

$\nabla$ Sec. 25.813

\begin{tabular}{||l|r|}
\hline \multicolumn{2}{|c|}{ Part 25 AIRWORTHINESS STANDARDS: TRANSPORT CATEGORY AIRPLANES } \\
\hline \hline Subpart D--Design and Construction & Emergency Provisions \\
\hline
\end{tabular}

Sec. 25.813

Emergency exit access.

Each required emergency exit must be accessible to the passengers and located where it will afford an effective means of evacuation. Emergency exit distribution must be as uniform as practical, taking passenger distribution into account; however, the size and location of exits on both sides of the cabin need not be symmetrical. If only one floor level exit per side is prescribed, and the airplane does not have a tailcone or ventral emergency exit, the floor level exit must be in the rearward part of the passenger compartment, unless another location affords a more effective means of passenger evacuation. Where more than one floor level exit per side is prescribed, at least one floor level exit per side must be located near each end of the cabin, except that this provision does not apply to combination cargo/passenger configurations. In addition--

(a) There must be a passageway leading from the nearest main aisle to each Type A, Type B, Type C, Type I, or Type II emergency exit and between individual passenger areas. Each passageway leading to a Type A or Type B exit must be unobstructed and at least 36 inches wide. Passageways between individual passenger areas and those leading to Type I, Type II, or Type C emergency exits must be unobstructed and at least 20 inches wide. Unless there are two or more main aisles, each Type A or B exit must be located so that there is passenger flow along the main aisle to that exit from both the forward and aft directions. If two or more main aisles are provided, there must be unobstructed crossaisles at least 20 inches wide between main aisles. There must be--

(1) A cross-aisle which leads directly to each passageway between the nearest main aisle and a Type A or B exit; and

(2) A cross-aisle which leads to the immediate vicinity of each passageway between the nearest main aisle and a Type I, Type II, or Type III exit; except that when two Type III exits are located within three passenger rows of each other, a single cross-aisle may be used if it leads to the vicinity between the passageways from the nearest main aisle to each exit.

(b) Adequate space to allow crewmember(s) to assist in the evacuation of passengers must be provided as follows:

(1) Each assist space must be a rectangle on the floor, of sufficient size to enable a crewmember, standing erect, to effectively assist evacuees. The assist space must not reduce the unobstructed width of the passageway below that required for the exit.

(2) For each Type A or B exit, assist space must be provided at each side of the exit regardless of whether an assist means is required by Sec. 25.810(a).

(3) For each Type C, I or II exit installed in an airplane with seating for more than 80 passengers, an assist space must be provided at one side of the passageway regardless of whether an assist means is required by Sec. 25.810(a).

(4) For each Type C, I or II exit, an assist space must be provided at one side of the passageway if an assist means is required by Sec. 25.810(a). 


\section{Table B.3: Regulations for Fuselage Sizing (continued)}

(5) For any tailcone exit that qualifies for 25 additional passenger seats under the provisions of [Sec. 25.807(g)(9)(ii)], an assist space must be provided, if an assist means is required by Sec. 25.810(a).

(6) There must be a handle, or handles, at each assist space, located to enable the crewmember to steady himself or herself:

(i) While manually activating the assist means (where applicable) and,

(ii) While assisting passengers during an evacuation.

(c) The following must be provided for each Type III or Type IV exit--

(1) There must be access from the nearest aisle to each exit. In addition, for each Type III exit in an airplane that has a passenger seating configuration of 60 or more--

(i) Except as provided in paragraph (c)(1)(ii), the access must be provided by an unobstructed passageway that is at least 10 inches in width for interior arrangements in which the adjacent seat rows on the exit side of the aisle contain no more than two seats, or 20 inches in width for interior arrangements in which those rows contain three seats. The width of the passageway must be measured with adjacent seats adjusted to their most adverse position. The centerline of the required passageway width must not be displaced more than 5 inches horizontally from that of the exit.

(ii) In lieu of one 10- or 20-inch passageway, there may be two passageways, between seat rows only, that must be at least 6 inches in width and lead to an unobstructed space adjacent to each exit.

(Adjacent exits must not share a common passageway.) The width of the passageways must be measured with adjacent seats adjusted to their most adverse position. The unobstructed space adjacent to the exit must extend vertically from the floor to the ceiling (or bottom of sidewall stowage bins), inboard from the exit for a distance not less than the width of the narrowest passenger seat installed on the airplane, and from the forward edge of the forward passageway to the aft edge of the aft passageway. The exit opening must be totally within the fore and aft bounds of the unobstructed space. (2) In addition to the access--

(i) For airplanes that have a passenger seating configuration of 20 or more, the projected opening of the exit provided must not be obstructed and there must be no interference in opening the exit by seats, berths, or other protrusions (including any seatback in the most adverse position) for a distance from that exit not less than the width of the narrowest passenger seat installed on the airplane.

(ii) For airplanes that have a passenger seating configuration of 19 or fewer, there may be minor obstructions in this region, if there are compensating factors to maintain the effectiveness of the exit.

(3) For each Type III exit, regardless of the passenger capacity of the airplane in which it is installed, there must be placards that--

(i) Are readable by all persons seated adjacent to and facing a passageway to the exit;

(ii) Accurately state or illustrate the proper method of opening the exit, including the use of handholds; and

(iii) If the exit is a removable hatch, state the weight of the hatch and indicate an appropriate location to place the hatch after removal.

(d) If it is necessary to pass through a passageway between passenger compartments to reach any required emergency exit from any seat in the passenger cabin, the passageway must be unobstructed. However, curtains may be used if they allow free entry through the passageway.

(e) No door may be installed between any passenger seat that is occupiable for takeoff and landing and any passenger emergency exit, such that the door crosses any egress path (including aisles, crossaisles and passageways).

(f) If it is necessary to pass through a doorway separating any crewmember seat (except those seats on the flightdeck), occupiable for takeoff and landing, from any emergency exit, the door must have a means to latch it in the open position. The latching means must be able to withstand the loads imposed upon it when the door is subjected to the ultimate inertia forces, relative to the surrounding structure, listed in Sec. 25.561(b). 
DANIEL NEUFELD

13 Autumn Place

Virgil, Ontario

LOS-1T0 Canada

\section{Education}

2005- RYERSON UNIVERSITY

PhD Candidate

2005 RYERSON UNIVERSITY

Master of Applied Science in Mechanical Engineering, April 2005

2003 RYERSON UNIVERSITY

Bachelor of Engineering in Aerospace Engineering, April 2003

\section{Honors and Rewards}

2004-2005 Ontario Graduate Scholarship

2003-2009 Ryerson Graduate Scholarship

1999-2003 Dean's List

\section{Research Interests}

Multi-disciplinary Design Optimization

Aircraft Conceptual Design

Reliability Based Design Optimization

\section{Teaching Assistant at Ryerson University}

I have held teaching assistant positions in the following courses at Ryerson University. My duties have included lab instruction, tutorial lectures, and marking
AER-416 Flight Mechanics
AER-520 Stress Analysis
AER-615 Aircraft Performance
AER-621 Aerospace Structural Design
AER-622 Gas Dynamics
AER-716 Aircraft Stability and Control
AER-814 Aircraft Design Project 
MEC-222 Engineering Graphical Communication

Internships

May-August 2002 Bombardier Aerospace, Toronto, Ontario

My duties as a summer intern at Bombardier included analyzing competitor aircraft data for developing performance comparisons used in marketing materials.

\section{Other Experience}

2010 Session chair at the 2010 CSME forum in Victoria, BC.

\section{List of Publications}

[1] Daniel Neufeld, Kamran Behdinan, and Joon Chung. Aircraft wing box optimization considering uncertainty in surrogate models. Structural and Multidisciplinary Optimization, July 2010.

[2] Martin Huber, Daniel Neufeld, Joon Chung, Horst Baier, and Kamran Behdinan. Data mining based mutation function for engineering problems with mixed continuous-discrete design variables. Structural and Multidisciplinary Optimization, 41(4):589-604, April 2010.

[3] Daniel Neufeld, Joon Chung, and Kamran Behdinan. Considering uncertain analysis methods in aircraft conceptual design optimization. In Proceedings of the 2010 CSME Forum, Victoria, BC, June 2010.

[4] Daniel Neufeld, Joon Chung, and Kamran Behdinan. Aircraft conceptual design optimization with uncertain contributing analyses. In Proceedings of the AIAA Modeling and Simulation Technologies Conference, Chicago, Illinois, August 2009.

[5] Daniel Neufeld, Kamran Behdinan, and Joon Chung. Development of an MDO platform for aircraft conceptual design. In Proceedings of the 2009 CANCAM Conference, Halifax, June 2009.

[6] D. Neufeld, J. Chung, and K. Behdinan. An approach to Multi-Objective aircraft design. Future Application and Middleware Technology on e-Science, pages 103-112, 2009.

[7] Daniel Neufeld, Joon Chung, and Behdinan Kamran. Development and application of MultiDisciplinary optimization software for aircraft conceptual design. International Review of Aerospace Engineering, 2008.

[8] D. Neufeld, J. Chung, and K. Behdinan. Development of a flexible MDO architecture for aircraft conceptual design. In Proceedings of the 2008 EngOpt conference. Rio de Jenario, Brazil, 2008.

[9] Daniel Neufeld and Joon Chung. Conceptual design optimization of very light jets. Proceedings of the 2007 CANCAM Conference, June 2007.

[10] Daniel Neufeld and Joon Chung. Enhancing UAV conceptual design using evolutionary algorithms and data mining. In Proceedings of the 2007 ICCSA Conference, 2007.

[11] Daniel Neufeld and Joon Chung. Unmanned aerial vehicle conceptual design using a genetic algorithm and data mining. In Proceedings of the Infotech@Aerospace Conference, September 2005. 